University of Rhode Island

DigitalCommons@URI

Open Access Dissertations

2017

\title{
Anthelmintic Efficacy of Bioactive Compounds Within Cranberry Vine and Birdsfoot Trefoil
}

Carly Barone

University of Rhode Island, carly_barone@my.uri.edu

Follow this and additional works at: https://digitalcommons.uri.edu/oa_diss

\section{Recommended Citation}

Barone, Carly, "Anthelmintic Efficacy of Bioactive Compounds Within Cranberry Vine and Birdsfoot Trefoil" (2017). Open Access Dissertations. Paper 599.

https://digitalcommons.uri.edu/oa_diss/599

This Dissertation is brought to you for free and open access by DigitalCommons@URI. It has been accepted for inclusion in Open Access Dissertations by an authorized administrator of DigitalCommons@URI. For more information, please contact digitalcommons-group@uri.edu. 


\author{
ANTHELMINTIC EFFICACY OF BIOACTIVE \\ COMPOUNDS WITHIN CRANBERRY VINE AND \\ BIRDSFOOT TREFOIL \\ BY \\ CARLY BARONE
}

A DISSERTATION SUBMITTED IN PARTIAL FULFILLMENT OF THE

REQUIREMENTS FOR THE DEGREE OF

DOCTOR OF PHILOSOPHY

IN

BIOLOGICAL AND ENVIRONMENTAL SCIENCES

UNIVERSITY OF RHODE ISLAND

2017 


\section{DOCTOR OF PHILOSOPHY DISSERTATION}

OF

CARLY BARONE

\section{APPROVED:}

Dissertation Committee:

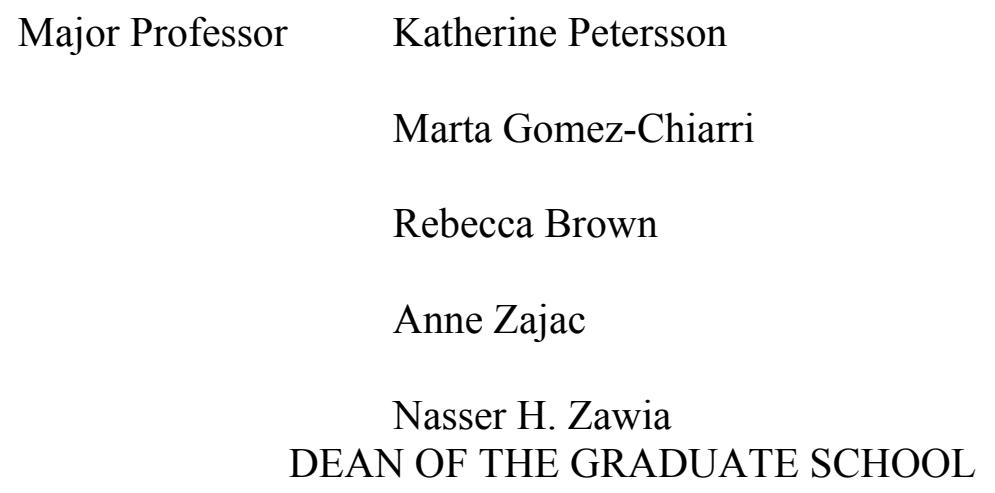

UNIVERSITY OF RHODE ISLAND

2017 


\begin{abstract}
With the growth of resistance to commercial dewormers in small ruminant gastrointestinal nematodes (GIN), alternative methods are needed for GIN control. It has been shown that plants containing certain secondary compounds (SC) suppress GIN infection, but there is a lack of knowledge concerning the mechanism behind this anthelmintic activity, as well as a lack of viable alternatives to commercial dewormers in the northeastern United States. Secondary compounds, such as proanthocyanidin (PAC), have complex chemical structures that vary between plant species, while SC concentrations can vary between different varieties of the same species. The relationship between specific SC and anti-parasitic efficacy is unknown. This study explores the use of PAC-rich cranberry vine $(\mathrm{CV})$ and birdsfoot trefoil (BFT) as an alternative to commercial dewormers in the control of GIN in small ruminants.

In vitro techniques were used to test BFT against $H$. contortus eggs, first stage (L1) and third stage larvae (L3). The anti-parasitic activity of purified proanthocyanidin extract (Bruce-PAC) and crude aqueous extracts (BFT-AqE) from 51 strains of BFT were tested using the following in vitro assays: 1) egg hatching and viability of L1 Haemonchus contortus larvae and 2) exsheathment and viability of L3 H. contortus larvae. The BFT-AqE was prepared by soaking BFT powder in water at room temperature for $24 \mathrm{~h}$, and then the plant matter was removed, leaving an aqueous extract. 1) For in vitro egg hatch inhibition and mortality of L1 larvae, H. contortus eggs were exposed to varying concentrations of BFT extracts for 24 h. 2) For in vitro exsheathment, 2,000 H. contortus L3 larvae were exposed to BFT extracts prior to exsheathment. Proanthocyanidin content ranged between 1.4 and $63.8 \mathrm{mg} \mathrm{PAC/g}$
\end{abstract}


powder across $51 \mathrm{BFT}$ strains. Inhibition of egg hatch and L1 mortality were observed: at $3 \mathrm{mg} / \mathrm{mL}$ BFT-AqE, percent inhibition of egg hatch and L1 mortality ranged between 0 and 100\% across 51 strains tested. Inhibition of exsheathment and L3 mortality was observed across thirteen tested strains: at $25 \mathrm{mg} / \mathrm{mL}$, percent inhibition of exsheathment bridged between 0 and $75 \%$ inhibition. Incubation in Bruce-PAC exhibited 8\% egg hatch inhibition, 98\% L1 mortality, 4\% percent L3 mortality, and $100 \%$ exsheathment inhibition at the highest concentration of $6 \mathrm{mg} / \mathrm{mL}$ Bruce-PAC. Results indicate that strains of BFT-AqE inhibited egg hatch and inhibited larval mortality, but the degree of inhibition varied. Results also indicate that Bruce-PAC did not inhibit egg hatch or mortality of L3 larvae, but did inhibit mortality of L1 larvae and inhibit exsheathment. When comparing PAC content of varietal BFT powders to anthelmintic efficacy of aqueous extracts, there were no significant correlations. Since PAC content was not related to activity, PAC structure was investigated for its potential influence on bioactivity. Further testing of PAC extracted from different varieties will provide additional information about the anthelmintic efficacy of strains of BFT for small ruminant GIN control. Inhibition of egg hatch, L1 and L3 mortality, and inhibition of exsheathment were observed with incubation in BFT-AqE; however, the concentration at which this inhibition was most effective varied among BFT strains. Correlations between the anthelmintic efficacy and PAC structure of each individual variety were determined based on these results. This study tested CV extracts against $H$. contortus eggs, first stage larvae, third stage larvae, and exsheathment. This investigation is the first to examine the anthelmintic potential of cranberry vine against the GIN Haemonchus contortus, while 
advancing the study of plants containing PAC. The purpose of this study was to explore the anti-parasitic potential of cranberry vine proanthocyanidin extract (CV$\mathrm{PAC})$ and cranberry vine aqueous extract (CV-AqE) on in vitro egg hatching, larval and adult worm mortality, and exsheathment of $H$. contortus, to observe cranberry vine treated $H$. contortus via scanning electron microscopy, and to investigate the efficacy of cranberry vine powder (CV) drench against an experimental infection of $H$. contortus in lambs. The in vitro anthelmintic effect on egg hatching and mortality of L1, L3, and adult worms was determined after 24-hour exposure to varying concentrations of CV-PAC and CV-AqE. The in vivo anthelmintic effect on an experimental infection in lambs was determined by administering $21.1 \mathrm{~g} \mathrm{CV}$ or not to lambs ( $n=9$ per group) for three consecutive days, and collecting fecal egg count (FEC) data for four weeks post treatment. Inhibition of egg hatch was observed in concentrations $\geq 5 \mathrm{mg} / \mathrm{mL} \mathrm{CV-PAC}$ and significant L1 mortality following hatching was observed at concentrations $\geq 0.3 \mathrm{mg} / \mathrm{mL}$. Egg hatch inhibition occurred at $\geq 5$ $\mathrm{mg} / \mathrm{mL}$ CV-AqE (0.6 mg PAC/mL), and significant L1 mortality occurred in L1 exposed to $\geq 1.2 \mathrm{mg} / \mathrm{mL}(0.15 \mathrm{mg} \mathrm{PAC} / \mathrm{mL})$. Third stage larvae exhibited mortality when exposed to $\geq 2.5 \mathrm{mg} / \mathrm{mL}$ CV-PAC; no significant mortality was observed in CVAqE. Inhibition of L3 exsheathment was observed at concentrations $\geq 5 \mathrm{mg} / \mathrm{mL} \mathrm{CV}$ PAC but was not observed at any concentration of CV-AqE. Adult worm mortality was observed at concentrations $\geq 0.6 \mathrm{mg} / \mathrm{mL} \mathrm{CV-PAC} \mathrm{and} \geq 12.5 \mathrm{mg} / \mathrm{mL} \mathrm{CV-AqE}$ (1.5 mg PAC $/ \mathrm{mL})$. Scanning electron microscopy revealed an accumulation of aggregate on the cuticle in the buccal area of adult worms incubated in both CV-AqE and CV-PAC. Both extracts (CV-PAC and CV-AqE) demonstrated anti-parasitic 
effects on all tested stages of $H$. contortus, but the extracts differed in their level of efficacy. In the lamb trial, no significant difference emerged between the weekly average FEC of the groups, but a significant treatment over time effect was observed.

Cranberry vine extracts were also tested in vitro against $H$. contortus adults and morphological changes in the adults incubated in extracts were observed. The purpose of this study was to investigate the effect that feeding cranberry vine (CV) to lambs had on 1) an established infection of $H$. contortus in lambs, 2) the establishment of a trickle infection of $H$. contortus in lambs, and 3) the morphology of $H$. contortus adult worms that were exposed to CV in vivo as determined by scanning electron (SEM), transmission electron (TEM), and light microscopy. Lambs were experimentally infected with L3 larvae in two separate trials; 1) CV trial: 21 lambs, stratified into three groups $(n=7)$ based on gender and fecal egg count (FEC) once the infection (10,000 H. contortus) matured; and 2) CVP trial: 14 lambs, stratified into two groups $(\mathrm{n}=7)$ based on gender and twin separation, received trickle infection of $500 \mathrm{~L} 3 \mathrm{H}$. contortus 3 times/week for 3 weeks. For the $\mathrm{CV}$ trial, lambs were fed CV0 $(0 \mathrm{~g} \mathrm{CV}$, 250 g chopped alfalfa hay (AH)), CV100 (100 g CV, 150 g AH), or CV200 (200 g $\mathrm{CV}, 50 \mathrm{~g} \mathrm{AH}$ ). Fecal egg counts (FEC) were measured weekly for the duration of the trial. At the conclusion of the study, total worm burden was determined and five adult female $H$. contortus worms were collected from the abomasum of each lamb and preserved until viewed under SEM, TEM or light microscopy. There was no difference in FEC between CV0 and CV200. Evaluation of worms using SEM showed cracking in the cuticle of the adult worms from the CV200 group, compared to minimal or no cracking observed in CV0. Some accumulation of an aggregate was 
observed on worms collected from the CV200 group. Evaluation of vesicles internal to the microvilli lining of the lumen of the intestines worms using TEM showed a lower number of vesicles present in the worms collected from the CV200 group. For the CVP trial, lambs were fed CVP0 diet: 0 g CVP, 500 g grain; or CVP200 diet: 500 g CVP, $100 \mathrm{~g}$ grain for 8 weeks. Fecal egg counts (FEC) were measured weekly for the duration of the trial. At the conclusion of the study, total worm and larval burden were determined. There was no difference in FEC or total worm burden between CVP0 and CVP200 groups. Results indicate that feeding $200 \mathrm{~g}$ of CV to lambs experimentally infected with $H$. contortus for five weeks resulted in cuticular damage and possible malnutrition of the adult worms and may have potential as an antiparasitic against $H$. contortus. Further investigation feeding a higher intake of $\mathrm{CV}$ is warranted. 


\section{ACKNOWLEDGMENTS}

I would like to thank my major professor, Dr. Katherine Petersson, for her endless support and mentorship. Her guidance throughout my undergraduate and graduate careers has been invaluable, as she has encouraged me tremendously and provided me with opportunities and experiences that have helped shape me professionally and personally. I would also like to thank Dr. Anne Zajac, Dr. Marta Gomez-Chiarri, and Dr. Rebecca Brown for their guidance on various projects and motivating me to explore new directions within my field of research and most importantly, to think outside of the box!

This journey would not have been possible without the help of other researchers and scientists along the way. I would like to thank Dr. Jess Reed, Dr. Christian Krueger, and Dr. Rodrigo Feliciano, and Kathy Lowe for their patience while providing valuable methodological expertise.

I have been exceptionally fortunate to work with so many intellectual URI students along the way. Several of them have contributed significantly to this work and have made working in lab a true pleasure: Karalyn Lonngren, Liz Lamperelli, Allison Unger, Nick LeMay, Holly Williams, Ryleigh Mullens, Shelby Ashworth, Cortney Loughran, Marissa Brummett, Jackie Foley, Gabby Bonofiglio, and Sydney Day. I especially appreciate the guidance and help of Nick Miniter, who has been instrumental in all of the work involving animals and has contributed countless innovative and resourceful ideas to various projects. His dedication to the animals on the farm and his always willingness to help is truly inspiring. I'd also like to thank 
Fred Launer for always being a source of guidance and encouragement throughout both my undergraduate and graduate careers.

Many other people who have inspired or supported me throughout my graduate journey include Laura Manzi, Kelly Monson, Danielle Johnson, Elder Gonzalez, Dr. Justin Richard, Dr. Becky Sartini, Dr. Bomi Sohn, Dr. Christopher Card. Last but definitely not least, thank you to the people who are always there to lean on, for their unconditional love and support: Brittani, Jess, Ashley, my Aunt Dee, my Aunt Sue, my Mémère, and most of all, my parents. 


\section{PREFACE}

This thesis is written in Manuscript Format. Chapter 1 includes a literature review of detailed information about small ruminant gastrointestinal nematodes, secondary plant metabolites and current proanthocyanidin research. Chapter 2 includes a condensed paper about birdsfoot trefoil in vitro testing. Chapter 3 includes a condensed paper about cranberry vine in vitro testing. Chapter 4 includes a condensed paper about cranberry vine in vivo testing. All manuscripts are written in the style of the journal Veterinary Parasitology. Conclusions made from all 3 studies are described in Chapter 5. Additional details on the methods used throughout these studies are included in the appendix. 


\section{TABLE OF CONTENTS}

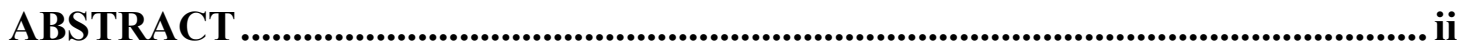

ACKNOWLEDGMENTS ................................................................................... vii

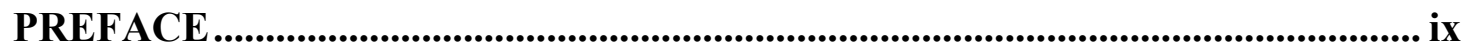

TABLE OF CONTENTS....................................................................................... $\mathrm{x}$

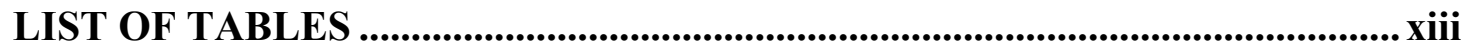

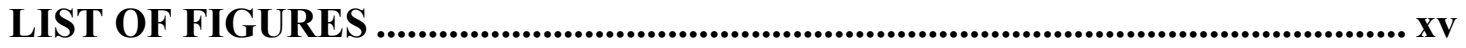

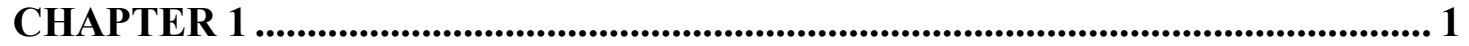

Introduction and Review of Literature .......................................................... 1

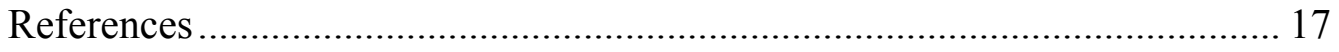

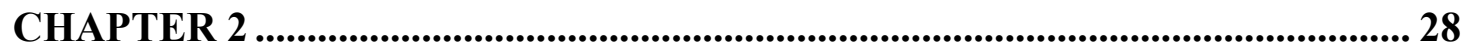

In vitro screening of fifty-one birdsfoot trefoil (Lotus corniculatus) strains for anthelmintic efficacy against Haemonchus contortus ....................................... 28

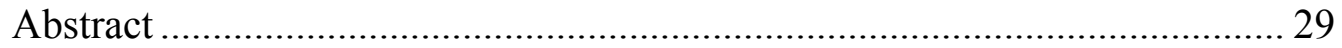

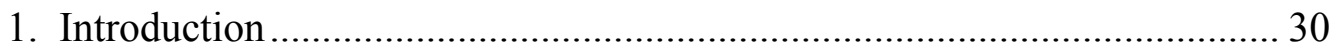

2. Materials and Methods ........................................................................ 32

3. Results ....................................................................................... 40

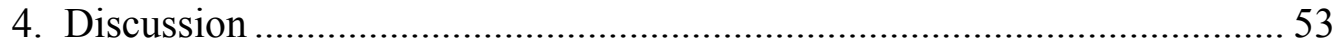

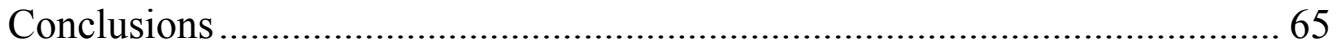

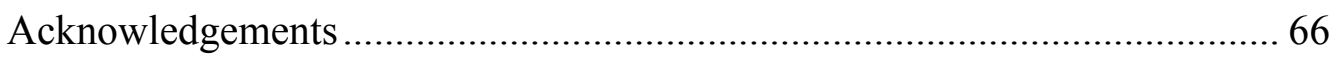

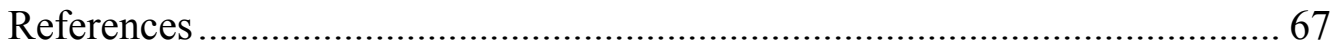


Anthelmintic efficacy of cranberry vine aqueous and proanthocyanidin extract on

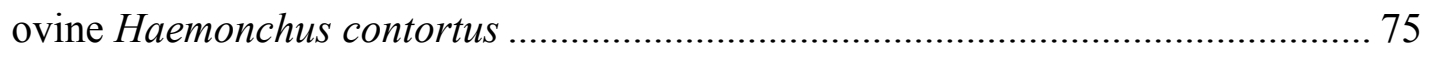

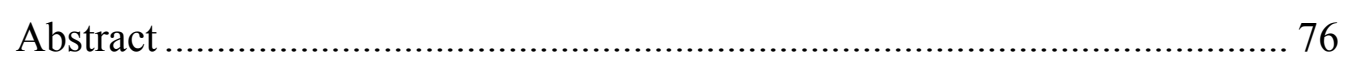

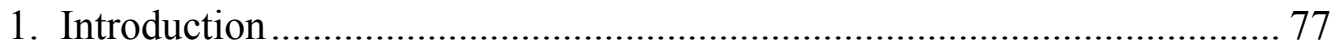

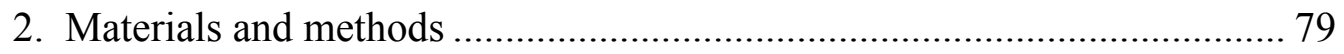

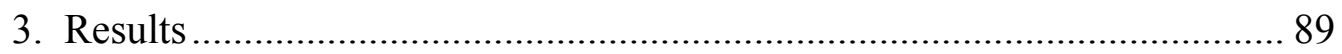

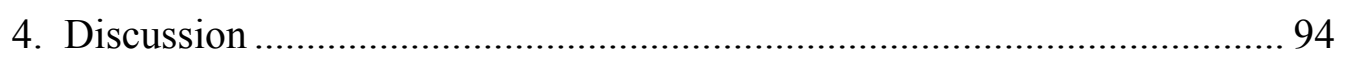

Conclusions ..................................................................................... 107

Acknowledgments.......................................................................... 108

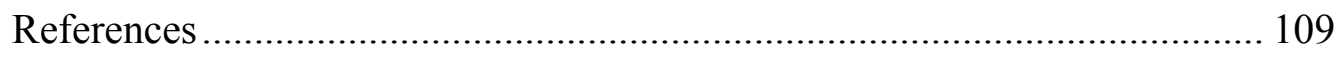

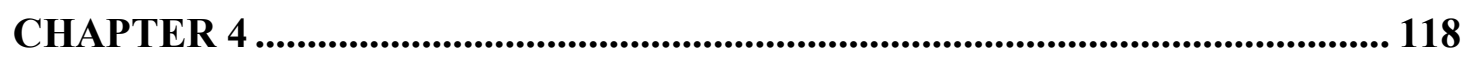

Effect of cranberry vine fed to lambs on experimental Haemonchus contortus

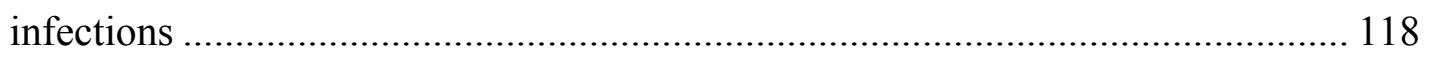

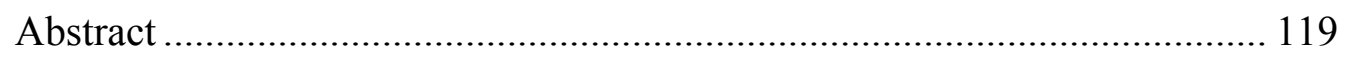

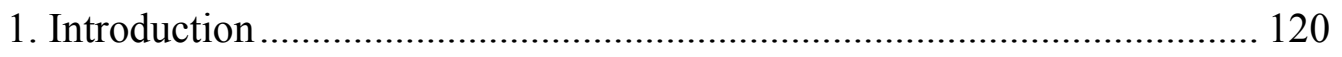

2. Materials and Methods .................................................................... 121

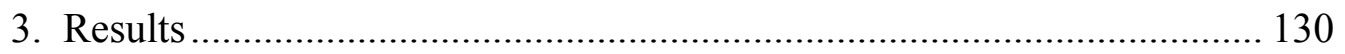

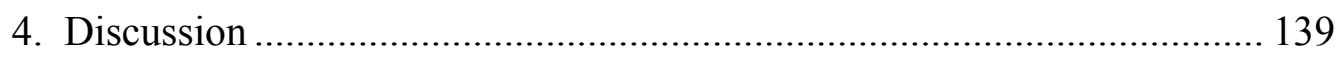

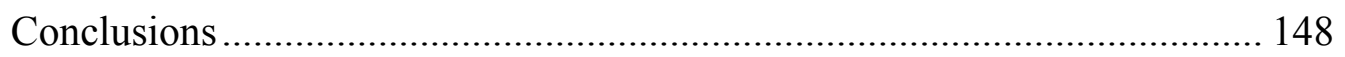

Acknowledgements ........................................................................ 148 


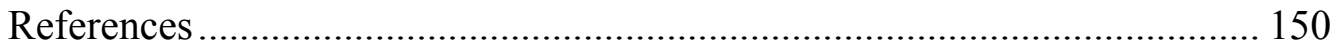

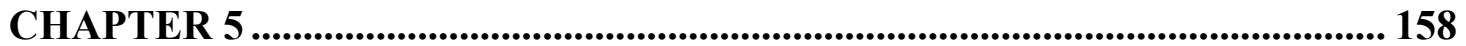

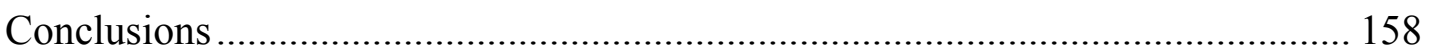

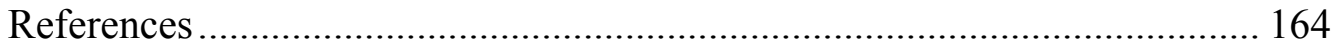

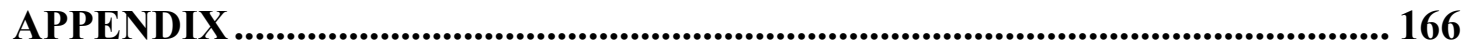

Standard Operating Procedure for Blood Collection ............................................. 166

Standard Operating Procedure for Nematode Recovery Post Mortem .................... 172

Standard Operating Procedure for Egg Recovery ................................................ 175

Standard Operating Procedure for Egg Hatch and L1 Mortality Assay.................. 177

Standard Operating Procedure for L3 Mortality and Exsheathment Inhibition Assay

References ........................................................................................................................ 185 


\section{LIST OF TABLES}

TABLE

PAGE

\section{Chapter 2}

Table 1. Seed suppliers of the 51 BFT strains used in the anthelmintic screening tests.

Table 2. Effect of 51 strains of birdsfoot trefoil (BFT) aqueous extracts and purified Bruce-PAC extract on Haemonchus contortus egg hatch and larval mortality posthatch. 42

Table 3. Lethal concentration that killed $50 \%\left(\mathrm{LC}_{50}\right)$ and $90 \%\left(\mathrm{LC}_{90}\right)(\mathrm{mg} / \mathrm{mL})$ and 95\% confidence interval $(95 \% \mathrm{CI})$ of BFT strains in Haemonchus contortus egg hatch inhibition test. 48

Table 4. Aqueous extracts of the top 13 BFT varieties and purified Bruce-PAC extract tested against Haemonchus contortus L3 mortality and exsheathment.

\section{Chapter 3}

Table 1. Effect of cranberry vine proanthocyanidin extract (CV-PAC) on Haemonchus contortus egg hatch and L1 mortality post-hatch, L3 mortality and exsheathment, and adult worm mortality. 90

Table 2. Effect of cranberry vine aqueous extract (CV-AqE) on Haemonchus contortus egg hatch and L1 mortality post-hatch, L3 mortality and exsheathment, and adult worm mortality. 95

Table 3. Lethal concentration that killed $50 \%\left(\mathrm{LC}_{50}\right)$ and $90 \%\left(\mathrm{LC}_{90}\right)(\mathrm{mg} / \mathrm{mL})$ and 95\% confidence interval $(95 \% \mathrm{CI})$ of CV-PAC and CV-AqE in Haemonchus contortus 
egg hatch inhibition, L1 mortality, L3 mortality, exsheathment inhibition, and adult

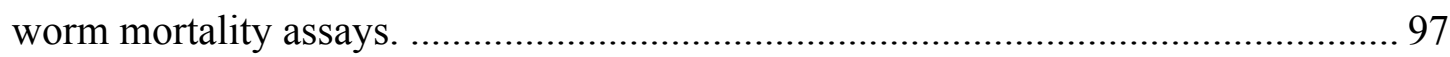




\section{LIST OF FIGURES}

FIGURE

PAGE

\section{Chapter 1}

Figure 1. Representative structure of a cranberry proanthocyanidin (PAC) trimer. ... 6

\section{Chapter 2}

Figure 1. The percent of egg hatch inhibition averages (mean $\pm \mathrm{SEM}$ ) and percent of larval mortality averages (mean $\pm \mathrm{SEM}$ ) in $6.0,3.0,1.5$, and $0.75 \mathrm{mg}$ powder/mL BFT-AqE.

Figure 2. Aqueous extracts (1.5 mg powder/mL) affect on egg hatching, L1 and L3 viability, and the exsheathment process compared to levels of PAC present in the BFT powders.

\section{Chapter 3}

Figure 1. Effect of cranberry vine proanthocyanidin (CV-PAC) and cranberry vine aqueous extract (CV-AqE) on the adult Haemonchus contortus worm through scanning electron microscopy. 98

Figure 2. Effect of cranberry vines (CV) on an experimental infection of Haemonchus contortus in lambs. 99

\section{Chapter 4}

Figure 1. CV Trial: Timeline of trial from -5 to 5 weeks, feeding chopped cranberry vine. 123

Figure 2. CVP Trial: Timeline of trial from 0 to 8 weeks, feeding pelleted cranberry vine. 125 
Figure 3. CV Trial: Effect of cranberry vines (CV) on fecal egg counts of an experimental infection of Haemonchus contortus in lambs.

Figure 4. CV Trial: Effect of cranberry vines (CV) on packed cell volume of an experimental infection of Haemonchus contortus in lambs.

Figure 5. Scanning electron microscopy of Haemonchus contortus worms exposed to control and cranberry vine (CV) diets in vivo. 134

Figure 6. Adult Haemonchus contortus cross-section viewed with light microscopy.

Figure 7. Transmission electron microscopy of Haemonchus contortus worms exposed to control and cranberry vine (CV) diets in vivo. 136

Figure 8. CVP Trial: Effect of cranberry vine pellet (CVP) on fecal egg counts of an experimental trickle infection of Haemonchus contortus in lambs. 138 


\section{CHAPTER 1}

Introduction and Review of Literature

\section{Gastrointestinal Nematodes in Small Ruminants}

Gastrointestinal nematodes (GIN) can result in economic and production losses for small ruminant producers worldwide, by causing anemia, poor body condition, diarrhea, and death. These trichostrongyle nematodes, (of the sub-order Trichostrongylina) persist as one of the leading limitations associated with pasturebased production of domestic small ruminants (Hoste et al., 2006). In 2015, the United States Department of Agriculture (USDA) reported 21,239 lambs and 13,543 sheep lost in the United States due to internal parasites (USDA, 2015). The gastrointestinal nematodes that account for the majority of economic and production loss are the barber pole worm (Haemonchus contortus), the brown stomach worm (Teladorsagia circumcincta), and the bankrupt worm (Trichostrongylus columbriformis).

Trichostrongyle nematodes (of the sub-order Trichostrongylina) are nematodes of the gastrointestinal tract and lungs (Hoste et al., 2006). Although the life cycles of these parasites are similar, they differ by predilection site and prepatent period. Adult GIN can reside in the abomasum, stomach, or small intestines of small ruminants, horses, or swine depending on the species. The infective L3 stage larvae, ingested by a small ruminant while grazing on pasture, undergo a process called exsheathment in the section of the GI tract proximal to the predilection site of that particular GIN species. Once the adult GIN is a mature female, it will lay eggs approximately two to 
three weeks after infecton. The length of the prepatent period is specific to the GIN species.

While the life cycles of GIN worms are similar, H. contortus (kingdom: Animalia, phylum: Nematoda, class: Secernentea, order: Strongylida, family: Trichostrongylidae, genus: Haemonchus, species: contortus) is the most pathogenic GIN, responsible for the majority of economic losses in sheep production worldwide (Veríssimo et al., 2012). This GIN species undergoes exsheathment in the first chamber of the ruminant stomach (rumen), where they lose their protective sheath. From the rumen they travel to the abomasum or true stomach where they mature into the adult worm. The adult worm resides in the abomasum, where it feeds on the blood of the host and mates, producing eggs that are shed through host defecation. Eggs are deposited on pasture in the feces of the animal, where they hatch into L1 stage larvae, which develop into L2 and L3 stage larvae before moving out of the fecal pellet onto pasture where they can be consumed by the small ruminant.

The pathogenicity of this GIN is the result of hemorrhage in the abomasum of heavily infected sheep (Ortolani et al., 2013). One adult nematode can remove up to $30 \mu \mathrm{l}$ of blood daily, thus in infections of $>500$ worms, anemia resulting in death can rapidly occur (Emery et al., 2016). Losses are most common in lambs and periparturient females, animals more susceptible to $H$. contortus infections (Santos et al., 2014) due to either the immature immune system of lambs (Besier, 2012) or suppression of the immune system by hormone levels and the metabolic demands of pregnancy (Vlassoff et. al., 2001). This susceptibility can lead to production losses and ultimately can be fatal. One female worm can yield between 5,000 and 15,000 
eggs per day (Emery et al., 2016); this can result in an overall output of 1.25 to 3.75 million eggs on pasture from an infection comprising of just 250 female worms.

\section{Parasite Control}

To address the rapid infection rates and production loss associated with $H$. contortus, modern commercial anthelmintic (deworming) drugs have been the preferred method used to control GIN infections due to their low cost and high efficacy. For the past 50 years, commercial dewormers were a great resource for small ruminant producers faced with GIN infections, but through their repeated use, there has been an emergence of parasite resistance to the dewormers (Hoste et al., 2006), creating a need to find alternative methods for GIN control. Plants rich in proanthocyanidins (PAC) and other secondary compounds (SC) have demonstrated anthelmintic activity against different species of GIN (Hoste et al., 2006). Previous studies have demonstrated an anthelmintic effect in sheep and goats consuming PAC containing forages (Keatinge, 1996; Min et al., 2004). One of the most heavily researched PAC forage effective in managing GIN infections is sericea lespedeza (Lespedeza cuneata) (Lange et al., 2006; Shaik et al., 2006). The New England climate is not suitable to sericea lespedeza, a tropical perennial. Many plants investigated for their anthelmintic properties cannot be grown in the harsh winter conditions of New England, making them impractical to use in the northeastern United States (Assis et al., 2003; Marie-Magdeleine et al., 2009).

\section{Tannins}

Tannins are secondary plant polyphenols, with a high affinity for proteins and polysaccharides. Tannins are produced by different plants and parts of the plants and 
are produced in particular seasons. Based on their structure, they are categorized into two major classes: proanthocyanidins (condensed tannins) and hydrolysable tannins (HT) (Reed, 1995). Hydrolysable tannins are gallic or ellagic acid esters of sugars and are more susceptible to hydrolysis than PAC and more soluble in water (Reed, 1995). When consumed by a ruminant, they degrade to gallic acid and can be absorbed in the gastrointenstinal tract. Because they are readily absorbed, hydrolysable tannins are known to be toxic to ruminants (Hoste et al., 2006) and therefore have not been extensively investigated for anthelmintic activity as proanthocyanidins have. Although published literature lacks information on the anthelmintic potential of hydrolysable tannins, it has been shown that HT-rich, or both PAC and HT-rich extracts were significantly more lethal to adult C. elegans than extracts containing only PAC (Katiki et al., 2013). While HT has been known for its toxicity, current literature actually indicates that ruminant consumption of large amounts of both PAC and HT may reduce feed digestibility and nitrogen retention, feed intake, fecal output, and cause toxicity, also indicating that neither HT nor PAC are toxic if consumed in moderation (Frutos et al., 2004; Hervás et al., 2003; Katiki et al, 2013).

\section{Proanthocyanidins}

Proanthocyanidins, more commonly referred to as condensed tannins, are the most common type of tannin found in forage legumes (Reed, 1995) and have a very complex structure. They are composed of oligomers and polymers of flavans, linked through single bonds (B-type) and a less common second ether linkage between an A-

ring of the lower unit and C-2 of the upper unit (A-type) (Howell, 2007) (Fig. 1). The specific structure of PAC varies depending on the plant species and variety, which 
influences biological activity. An example of a plant containing mostly (94.5\%) Atype PAC interflavan bonds is the cranberry plant (Feliciano et al., 2014), while forages such as perennial ryegrass and tall fescue, contain B-type PAC, with only low levels of A-type linkages present (Fraser et al., 2016). While perennial ryegrass and tall fescue are forage species of the monocot grass family, PAC has also been found in forage and grain legumes, shrubs and cereals (Fraser et al., 2016). Previous investigators have not reported A-type linkages in PAC from other monocot species; for example, barley and sorghum PAC presented solely B-type linkages (Fraser et al., 2016). An investigation of 39 PAC containing human consumed foods (fruits, vegetables, nuts, cereals/beans, and spices), found 21 of them to contain only B-type linkages and the rest of the foods had B-type bonds as the main component, containing only low levels of A-type PAC (Gu et al., 2003). Although B-type bonds are more common (Howell, 2007), studies have shown that the A-type bonds found in cranberries have greater bioactivity than the B-type PAC found in apples, specifically against Escherichia coli (Feliciano et al., 2014).

Proanthocyanidins are found in the stems, leaves, flowers, fruits, roots, and seeds of plants. The water-soluble PAC is concentrated in the vacuole, primarily near the vaculor membrane or tonoplast (Abeynayake et al., 2011). Investigators studying the accumulation of PAC in birdsfoot trefoil (Lotus corniculatus) plant tissues, found PAC accumulation to be higher in more mature plants (Abeynayake et al., 2011) and found PAC concentration increased as plants matured (Cassida et al., 2000; Grabber et al., 2014). This is important when considering the use of feeding PAC-containing forages as a GIN control method. The timing of harvest affects PAC concentration, as 
well as the method of harvest. In the case of harvesting a PAC-containing forage as hay or harvesting to create a pellet, the temperature used in the drying processes and

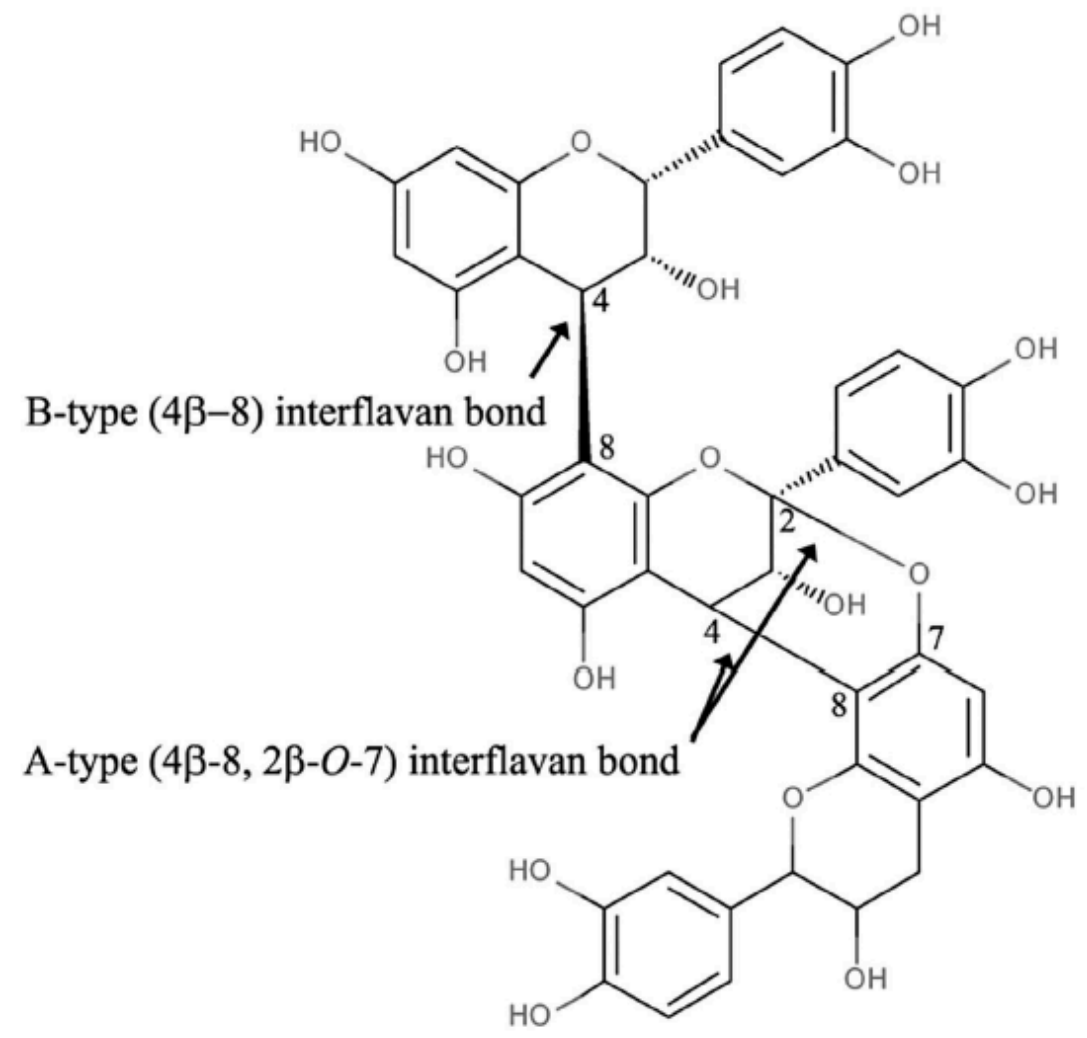

Figure 1. Representative structure of a cranberry proanthocyanidin (PAC) trimer. Variation in degree of polymerization, position, and number of "Atype" versus "B-type" interflavan bonds leads to large structural heterogeneity among cranberry PAC. Image from published study by Krueger et al. (2016).

pelleting processes must be considered. As a consequence of heating, proanthocyanidins can form covalent bonds with proteins through oxidative polymerization reactions (Reed, 1995). Since PAC has the potential to complex under high heat conditions, it could potentially change the structure of PAC and ultimately alter its bioactivity (Terrill et al., 2007).

The majority of published work investigating the anti-parasitic effect of feeding PAC rich plants has looked at sulla (Hedysarum coronarium), sainfoin 
(Onobrychis viciifolia), birdsfoot trefoil (Lotus corniculatus), big trefoil (Lotus pedunculatus), sericea lespedeza (Lespedeza cuneata), and chicory (Cichorium intybus) (Hoste et al., 2006). In vitro testing of PAC against GIN has included testing against eggs, larvae, the exsheathment process and adult worms. In vitro testing against egg hatching, has confirmed that the hatch rate of GIN eggs was lower in sheep fed PAC rich plants compared to control groups fed PAC free diets (Niezen et al., 2002). Proanthocyanidins are not absorbed from the lumen of the gastrointestinal tract, (Terrill et al., 1994) therefore, they are found in the feces of animals consuming PAC (Niezen et al., 2002). Wattle tannin, a commercially sold CT extract powder from the bark of Acacia mearnsii, was used to drench sheep and goats that had naturally acquired infections and feces were then cultured from these animals. Max (2010) determined that the drench was capable of reducing the hatchability of nematode eggs in the manure.

Although the effect of PAC on egg hatching has been documented, there has been variability between different PAC containing extracts. Vargas-Magaña et al. (2014) tested egg hatch inhibition in vitro using four PAC containing plant extracts (Lysiloma latisiliquum, Laguncularia racemosa, Rizophora mangle, Avicennia germinans); they observed one extract that showed ovicidal activity at concentrations as low as $0.6 \mathrm{mg} / \mathrm{mL}$ and another extract that had no efficacy at the highest concentration of $3.6 \mathrm{mg} / \mathrm{mL}$. Another study investigating egg hatch inhibition, using PAC extracts from five species of plants (Lotus pedunculatus, Lotus corniculatus, Dorycnium pentaphyllum, Dorycnium rectum, Rumex obtusifolius), reported between $46 \%$ and $68 \%$ of eggs hatched (across the five plant extracts) in $0.9 \mathrm{mg} / \mathrm{mL}$ of PAC 
(Molan and Faraj, 2010).

The anthelmintic effects reported in previous studies confirmed anti-parasitic activity, but the mechanism of how PAC exerts injury on the nematode is unknown. Studies have shown that PAC has the ability to bind to proteins either by hydrogen bonding between tannins and protein, or via covalent bonding to proteins after oxidization (Taiz and Zeiger, 2006). Consumption of large quantities of PAC can have negative impacts on an animals' ability to utilize protein. These negative effects are associated with protein binding, including inactivation of digestive enzymes creating complex tannin-protein aggregates that are hard to digest (Taiz and Zeiger, 2006). While there are negative impacts associated with consuming high levels of tannins, there have been positive health benefits associated with low or moderate tannin intake. Aside from the effects that PAC has on parasitic infections, other beneficial effects have been observed in ruminants consuming low or moderate concentrations of PAC ( $<6 \%$ of DM), such as increased wool and milk production and increased growth (Hoste et al., 2006).

\section{Suspected mechanisms of action}

There are currently two main hypotheses that have been proposed to explain the effect of PAC on small ruminant GIN. The first hypothesis is that PAC is improving the immune response of the host to control the infection (Hoste et al., 2006). This hypothesis states that an increase in intestinal protein, caused by the ability of PAC to bind to proteins and protect them from degradation in the rumen, increases protein availability in the small intestine. The ability of PAC to bind to proteins has been termed "by-pass protein" and results in a greater supply of amino 
acids to the small intestine, ultimately improving immune function (Waghorn, 1996). An improved immune system may benefit the overall health of the animals and enhance the animals' immune response to a GIN infection (Hoste et al., 2006). The second hypothesis is that PAC disturbs biological processes of the nematode directly. This direct hypothesis is supported by the anthelmintic efficacy observed under in vitro conditions, and is explained by the assumption that PAC is able to bind to the protein-rich nematode cuticle (Hoste et al., 2006). Investigators of the morphological changes in the worm exposed to PAC have used techniques including scanning and transmission electron microscopy to observe physical changes. Physical changes include the accumulation of aggregate on the nematode cuticle (Hoste et al., 2006). These procedures include a critical point drying step, possibly introducing significant morphological artifacts (Paterson et al, 2013). During critical point drying, water in the sample is replaced by a transitional fluid (acetone), which is then replaced by liquid $\mathrm{CO}_{2}$. The sample is heated to $31^{\circ} \mathrm{C}$ at which point $\mathrm{CO}_{2}$ becomes supercritical (Paterson et al, 2013). If the temperature required to reach supercritical conditions is above the glass transition point of the sample, then it may melt, causing the native morphology to be destroyed or distorted (Paterson et al, 2013). When viewing the morphology of worms prepared in this way, this must be taken into consideration. Although there are studies investigating these hypotheses, the mechanisms driving the efficacy of proanthocyanidins remain undetermined.

Secondary Compounds (SC)

Proanthocyanidins are secondary plant compounds. Secondary compounds are a large, diverse group of organic compounds that have no direct function in growth 
and development (Taiz and Zeiger, 2006). Secondary compounds in an individual plant are dependent on developmental stage, environment, and genetics (Cheynier et al., 2012). Most plants produce a wide range of secondary compounds either as defense compounds against pathogens and herbivores or in response to abiotic stresses, including radiation, cold temperatures, water stress, and nutrient deficiency (Cheynier et al., 2012). Extractions of secondary compounds include organic extractions and aqueous extractions. Extracts of plants can contain many different SC, including PAC, but are not limited to PAC. Proanthocyanidins are not the only group of naturally occurring plant compounds that have been investigated for their anthelmintic potential. Anti-parasitic effects of aqueous extracts of different plants have been demonstrated against egg hatching, larvae, and adult worms of nematodes infecting animals (Adamu et al., 2010; Ferreira et al., 2013) and have demonstrated anthelmintic efficacy against nematodes infecting humans (Klimpel et al., 2011). These investigations attributed the anti-parasitic effect of the plant to various SC including alkaloid, saponin, and phenolic compounds.

Aqueous extractions versus organic extractions

The majority of studies investigating the anthelmintic efficacy of plant extracts use crude organic extracts (Acharya et al., 2014; von Son-de Fernex et al., 2012; Klongsiriwet et al., 2015), one example being methanol extraction (Acharya et al., 2014). In vitro testing using plant derived PAC organic extracts has demonstrated inhibition of egg hatch against $H$. contortus (Vargas-Magaña et al., 2014) and inhibited larval development against Teladorsagia circumcincta (Molan and Faraj, 2010). These investigations have concluded that there are other secondary compounds 
present in crude organic extracts, besides PAC, which may also be playing a role in the anthelmintic efficacy observed (Hernández-Villegas et al., 2011; Monteiro et al., 2011). Secondary metabolites, including proanthocyanidins, have been considered responsible for the anti-parasitic effect of plants as well as saponins, alkaloids, nonprotein amino acids, polyphenols, lignins and glycosides (Githiori et al., 2006).

Other studies using crude aqueous extracts soak plant-derived products in water (Adamu et al., 2010; Ferreira et al., 2013). Crude aqueous extracts showing anthelmintic efficacy against $H$. contortus attributed this activity to alkaloids, saponins, (Adamu et al., 2010) and phenolic compounds (Ferreira et al., 2013). An example of a crude aqueous leaf extract of Annona muricata L. (Annonaceae) (50\% dilution) was prepared by infusing $100 \mathrm{~g}$ fresh leaves in $1 \mathrm{~L}$ boiling water. Crude aqueous extracts of plants tested against small ruminant strongylid nematode egg hatching and larval growth showed anti-parasitic effects (Adamu et al., 2010), while crude aqueous extracts tested against $H$. contortus eggs, larvae and adult worms, also showed anthelmintic efficacy (Ferreira et al., 2013).

The mechanism of extraction is a major difference between aqeuous and organic extracts. Organic solvents dissolve cell membranes and release both membrane-bound compounds such as chlorophyll and water-soluble compounds held within membranes. Aqeuous solvents cannot dissolve membranes, they can only extract when something else has ruptured the membranes. Examples of this include freeze-drying, grinding, and temperatures above $40^{\circ} \mathrm{C}$. Previous investigators using aqueous extracts ground the plant material first and then use boiling water to extract the secondary compounds (Adamu et al., 2010). As mentioned before heating 
proanthocyanidins can form covalent bonds with proteins through oxidative polymerization reactions (Reed, 1995). Since PAC has the potential to complex under high heat conditions, it could potentially change the structure of PAC and ultimately alter its bioactivity (Terrill et al., 2007).

In vitro investigations determining PAC content of plant material or extracts have commonly used the butanol-HCl (BuOH-HCl) method (Terrill et al., 1992; Lange et al., 2006). This method has been reported to include sources of interference, such as anthocyanins, while the 4-(dimethylamino)cinnamaldehyde (DMAC) method is more accurate and sensitive for PAC (Feliciano et al., 2012). Proanthocyanidin quantification using the DMAC method has recently been accepted as the method of choice for quantification of PAC by the cranberry industry (Krueger et al., 2013; Krueger et al., 2016). The cranberry industry is also currently promoting content of insoluble PAC. Therefore, because the DMAC method is only able to quantify soluble/extractable PAC, a subsequent analysis on powder residue following extraction for quantification of soluble $\mathrm{PAC}$, is run using the $\mathrm{BuOH}-\mathrm{HCl}$ method. Therefore, this type of PAC content determination reports quantification of extracted soluble PAC (using the DMAC method) and the residue (insoluble PAC) subjected to $\mathrm{BuOH}-\mathrm{HCl}$ analysis.

When analyzing PAC content in extracts or plant material, the use of a correct standard increases the accuracy of the results. The standard used for analyses must be reflective of the structural heterogeneity of plant investigated. For example when investigating cranberries, a c-PAC standard is commonly used by the cranberry industry as a suitable standard, instead of a procyanidin A2 (ProA2) standard (Krueger 
et al., 2013). The ProA2 standard was found to result in values that were 3.6-fold lower than determined by c-PAC (Krueger et al., 2016). This underestimation of PAC concentration (using ProA2 standards) was attributed to the fact that the ProA2 dimer is a single compound, while the c-PAC standard is a mixture of proanthocyanidins (dimers, trimers, tetramers, etc) and is better reflective of the polymerization, hydroxylation, and ratios of A-type to B-type interflavan bonds found in cranberry vine PAC (Krueger et al., 2016).

\section{Cranberry Vine (CV) Proanthocyanidin}

The American cranberry (Vaccinium macrocarpon) is a rich source of polyphenol compounds, including PAC, flavanols, anthocyanidins, and phenolic acids (Feliciano et al., 2016). Of these compounds, the most abundant is PAC, containing A-type PAC interflavan bonds (Feliciano et al., 2016). Native to the United States, cranberries are predominantly grown in Wisconsin, Massachusetts, New Jersey, Oregon and Washington (Polashock et al., 2014). The annual pruning of cranberry bogs, as the vines come out of winter dormancy, provides a readily available source of vine. The use of $\mathrm{CV}$ as a medicinal forage would benefit cranberry growers as well as livestock producers. Cranberry PAC extract has been investigated for its anti-bacterial properties and its role in maintaining urinary tract health (Howell et. al., 2005). The structure of A-linked dimers and trimers proved to be active in the anti-adhesion of bacteria, related to human urinary tract infections (Howell, 2007). Aside from cranberry PACs potential for benefiting urinary tract health, recent research has proposed that cranberry PAC can inhibit tumor cell growth (Neto et al., 2006), decrease risk of cardiovascular (Porter et al., 2001), gastrointestinal (caused by 
Helicobacter pylori) (Burger et al., 2000) and periodontal (Weiss et al., 2004)

diseases, and prevent disease caused by noroviruses (Su et al., 2010) (Feliciano et al., 2012).

\section{Birdsfoot Trefoil (BFT) Proanthocyanidin}

Investigated for its anthelmintic potential, birdsfoot trefoil (Lotus corniculatus) showed a reduction in FEC (Marley et al, 2003) and total worm burden in vivo (Heckendorn et. al., 2007; Marley et al, 2003). Birdsfoot trefoil is a drought resistant legume (Hedqvist, 2000), which tolerates low soil fertility and is adapted to the northeastern United States. Grazing this legume provides additional benefits to the animal by minimizing bloat and improving protein uptake (MacAdam and Griggs, 2006). This would prove environmentally beneficial, as the minimization of bloat would in turn decrease the rate of ruminal gas and reduce methane emissions (Puchala et. al., 2005). Improvement of pasture and forage quality can be achieved through nitrogen fixation, by growing BFT together or in rotation with grasses. There are numerous varieties of BFT, which differ in biochemical, morphological, and agronomic characteristics (Steiner et al., 2001). A study investigating morphological and chemical characteristics of $14 \mathrm{BFT}$ varieties found PAC concentrations to differ substantially among BFT cultivars (Grabber et al., 2014).

Previous studies (Roberts et al. 1993, Steiner et al. 2001) have characterized total PAC levels in BFT germplasm using near-infrared reflectance spectroscopy (NIRS). However, the degree of correlation between anthelmintic activity and total PAC is unknown. This presents a need to investigate the relationship between antiparasitic activity and PAC levels and structures found in the strains of BFT. 
Characterization of tannin levels using modern methods of PAC analysis, such as the DMAC assay has improved accuracy and reduced interference by other polyphenols (anthocyanins, flavonols and hydroxycinnamic acids) compared to the older published assays (Feliciano et al., 2012). These findings demonstrate the need to more fully characterize PAC profiles of BFT strains and identify the bioactive components in order to select strains that provide consistent results.

Potential of $C V$ and BFT as anthelmintics

Cranberry vine and BFT as anthelmintics have the potential to provide natural alternatives to commercial dewormers, which could potentially, decrease producer reliance on current commercial dewormers. A reduced reliance on commercial dewormers would be environmentally beneficial for the soil microorganisms that aid in pasture productivity and nitrogen fixation (Ollivier et al., 2011). For example, studies investigating the effects of macrocyclic lactones (commercial dewormers) on dung-dwelling insects, observed reduced feeding and lower reproductive rates, which decreased dung beetle populations and eventually decreased rates of dung degradation and dung burial (Jacobs and Scholtz, 2014). The GIN health concerns associated with immunologically naïve lambs on pasture would also be addressed by these natural anthelmintics. Reducing health risks like GIN infections would ultimately improve productivity due to lower worm burden, as well as increase the net farm income in conjunction with a decreased use of commercial dewormers. This study also has the potential to further our understanding of how plant compounds exert their anthelmintic effect on $H$. contortus, what type of interaction is taking place between the $\mathrm{SC}$ and the parasite itself, and which SC are causing these effects. This knowledge will aid in the 
effective screening of plants for true anthelmintic potential and may offer the northeastern United States a crop that can be used as part of an integrated parasite control program for small ruminants. 


\section{References}

Abeynayake, S.W., Panter, S., Mouradov, A., Spangenberg, G., 2011. A highresolution method for the localization of proanthocyanidins in plant tissues. Plant methods 7 (1), 13.

Acharya, J., Hildreth, M.B., Reese, R.N., 2014. In vitro screening of forty medicinal plant extracts from the United States Northern Great Plains for anthelmintic activity against Haemonchus contortus. Vet. Parasitol. 201 (1), 75-81.

Adamu, M., Oshadu, O.D., Ogbaje, C.I., 2010. Anthelminthic efficacy of aqueous extract of Acanthus montanus leaf against strongylid nematodes of small ruminants. Afr. J. Tradit. Complement. Altern. Med. 7 (4), 279-285.

Assis, L.M., Bevilaqua, C.M.L., Morais, S.M., Vieira, L.S., Costa, C.T.C., Souza, J.A.L., 2003. Ovicidal and larvicidal activity in vitro of Spigelia anthelmia Linn. extracts on Haemonchus contortus. Vet. Parasitol. 117 (1-2), 43-49, http://dx.doi.org/10.1016/j.vetpar.2003.07.021.

Besier, R.B., 2012. Refugia-based strategies for sustainable worm control: Factors affecting the acceptability to sheep and goat owners. Vet. Parasitol. 186 (1-2), 2-9, http://dx.doi.org/10.1016/j.vetpar.2011.11.057.

Burger, O., Ofek, I., Tabak, M., Weiss, E.I., Sharon, N., Neeman, I., 2000. A high molecular mass constituent of cranberry juice inhibits helicobacter pylori adhesion to human gastric mucus. FEMS Immunol. Med. Microbiol. 29 (4), 295-301, S0928-8244(00)00220-0 [pii]. 
Cassida, K., Griffin, T., Rodriguez, J., Patching, S., Hesterman, O., Rust, S., 2000. Protein degradability and forage quality in maturing alfalfa, red clover, and birdsfoot trefoil. Crop Sci. 40 (1), 209-215.

Cheynier, V., Sarni-Manchado, P., Quideau, S., 2012. Recent Advances in Polyphenol Research. John Wiley \& Sons.

Emery, D.L., Hunt, P.W., Le Jambre, L.F., 2016. Haemonchus contortus: the then and now, and where to from here? Int. J. Parasitol. 46 (12), 755-769, S00207519(16)30178-3 [pii].

Feliciano, R.P., Shea, M.P., Shanmuganayagam, D., Krueger, C.G., Howell, A.B., Reed, J.D., 2012. Comparison of isolated cranberry (Vaccinium macrocarpon Ait.) proanthocyanidins to catechin and procyanidins A2 and B2 for use as standards in the 4-(dimethylamino) cinnamaldehyde assay. J. Agric. Food Chem. 60 (18), 4578-4585.

Feliciano, R.P., Boeres, A., Massacessi, L., Istas, G., Ventura, M.R., Nunes Dos Santos, C., Heiss, C., Rodriguez-Mateos, A., 2016. Identification and quantification of novel cranberry-derived plasma and urinary (poly)phenols. Arch. Biochem. Biophys. 599, 31-41, 10.1016/j.abb.2016.01.014 [doi].

Feliciano, R.P., Meudt, J.J., Shanmuganayagam, D., Krueger, C.G., Reed, J.D., 2014. Ratio of "A-type" to "B-type" proanthocyanidin interflavan bonds affects extra-intestinal pathogenic Escherichia coli invasion of gut epithelial cells. J. Agric. Food Chem. 62 (18), 3919-3925, 10.1021/jf403839a [doi].

Ferreira, L.E., Castro, P.M., Chagas, A.C., Franca, S.C., Beleboni, R.O., 2013. In vitro anthelmintic activity of aqueous leaf extract of Annona muricata $\mathrm{L}$. 
(Annonaceae) against Haemonchus contortus from sheep. Exp. Parasitol. 134 (3), 327-332, 10.1016/j.exppara.2013.03.032 [doi].

Fraser, K., Collette, V., Hancock, K.R., 2016. Characterization of Proanthocyanidins from Seeds of Perennial Ryegrass (Lolium perenne L.) and Tall Fescue (Festuca arundinacea) by Liquid Chromatography-Mass Spectrometry. J. Agric. Food Chem. 64 (35), 6676-6684, 10.1021/acs.jafc.6b02563 [doi].

Frutos, P., Raso, M., Hervás, G., Mantecón, Á.R., Pérez, V., Giráldez, F.J., 2004. Is there any detrimental effect when a chestnut hydrolysable tannin extract is included in the diet of finishing lambs? Animal Research 53 (2), 127-136.

Githiori, J.B., Athanasiadou, S., Thamsborg, S.M., 2006. Use of plants in novel approaches for control of gastrointestinal helminths in livestock with emphasis on small ruminants. Vet. Parasitol. 139 (4), 308-320, http://dx.doi.org/10.1016/j.vetpar.2006.04.021.

Grabber, J., Riday, H., Cassida, K., Griggs, T., Min, D., MacAdam, J., 2014. Yield, morphological characteristics, and chemical composition of European-and Mediterranean-derived birdsfoot trefoil cultivars grown in the colder continental United States. Crop Sci. 54 (4), 1893-1901.

Gu, L., Kelm, M.A., Hammerstone, J.F., Beecher, G., Holden, J., Haytowitz, D., Prior, R.L., 2003. Screening of foods containing proanthocyanidins and their structural characterization using LC-MS/MS and thiolytic degradation. J. Agric. Food Chem. 51 (25), 7513-7521, 10.1021/jf034815d [doi].

Heckendorn, F., Haring, D.A., Maurer, V., Senn, M., Hertzberg, H., 2007. Individual administration of three tanniferous forage plants to lambs artificially infected 
with Haemonchus contortus and Cooperia curticei. Vet. Parasitol. 146 (1-2), 123-134, S0304-4017(07)00052-0 [pii].

Hedqvist, H., Mueller-Harvey, I., Reed, J.D., Krueger, C.G., Murphy, M., 2000. Characterisation of tannins and in vitro protein digestibility of several Lotus corniculatus varieties. Anim. Feed Sci. Technol. 87 (1), 41-56.

Hernández-Villegas, M.M., Borges-Argáez, R., Rodriguez-Vivas, R.I., Torres-Acosta, J.F.J., Méndez-Gonzalez, M., Cáceres-Farfan, M., 2011. Ovicidal and larvicidal activity of the crude extracts from Phytolacca icosandra against Haemonchus contortus. Vet. Parasitol. 179 (1-3), 100-106, http://dx.doi.org/10.1016/j.vetpar.2011.02.019.

Hervás, G., Pérez, V., Giráldez, F.J., Mantecón, A., Almar, M., Frutos, P., 2003. Intoxication of sheep with quebracho tannin extract. J. Comp. Pathol. 129 (1), $44-54$.

Hoste, H., Jackson, F., Athanasiadou, S., Thamsborg, S.M., Hoskin, S.O., 2006. The effects of tannin-rich plants on parasitic nematodes in ruminants. Trends Parasitol. 22 (6), 253-261, S1471-4922(06)00099-7 [pii].

Howell, A.B., 2007. Bioactive compounds in cranberries and their role in prevention of urinary tract infections. Mol. Nutr. Food Res. 51 (6), 732-737, 10.1002/mnfr.200700038 [doi].

Howell, A.B., Reed, J.D., Krueger, C.G., Winterbottom, R., Cunningham, D.G., Leahy, M., 2005. A-type cranberry proanthocyanidins and uropathogenic bacterial anti-adhesion activity. Phytochemistry 66 (18), 2281-2291, S00319422(05)00249-9 [pii]. 
Jacobs, C.T., Scholtz, C.H., 2015. A review on the effect of macrocyclic lactones on dung-dwelling insects: Toxicity of macrocyclic lactones to dung beetles. Onderstepoort J. Vet. Res. 82 (1), 858, 10.4102/ojvr.v82i1.858 [doi].

Katiki, L.M., Ferreira, J.F., Gonzalez, J.M., Zajac, A.M., Lindsay, D.S., Chagas, A.C.S., Amarante, A.F., 2013. Anthelmintic effect of plant extracts containing condensed and hydrolyzable tannins on Caenorhabditis elegans, and their antioxidant capacity. Vet. Parasitol. 192 (1), 218-227.

Keatinge, R., 1996. Case studies in homeopathy. New Farmer and Grower (United Kingdom).

Klimpel, S., Abdel-Ghaffar, F., Al-Rasheid, K.A., Aksu, G., Fischer, K., Strassen, B., Mehlhorn, H., 2011. The effects of different plant extracts on nematodes. Parasitol. Res. 108 (4), 1047-1054.

Klongsiriwet, C., Quijada, J., Williams, A.R., Mueller-Harvey, I., Williamson, E.M., Hoste, H., 2015. Synergistic inhibition of Haemonchus contortus exsheathment by flavonoid monomers and condensed tannins. Int. J. Parasitol. Drugs Drug Resist 5 (3), 127-134, 10.1016/j.ijpddr.2015.06.001 [doi].

Krueger, C.G., Chesmore, N., Chen, X., Parker, J., Khoo, C., Marais, J.P., Shanmuganayagam, D., Crump, P., Reed, J.D., 2016. Critical reevaluation of the 4-(dimethylamino) cinnamaldehyde assay: cranberry proanthocyanidin standard is superior to procyanidin A2 dimer for accurate quantification of proanthocyanidins in cranberry products. Journal of Functional Foods 22, 1319. 
Krueger, C.G., Reed, J.D., Feliciano, R.P., Howell, A.B., 2013. Quantifying and characterizing proanthocyanidins in cranberries in relation to urinary tract health. Analytical and bioanalytical chemistry 405 (13), 4385-4395.

Lange, K.C., Olcott, D.D., Miller, J.E., Mosjidis, J.A., Terrill, T.H., Burke, J.M., Kearney, M.T., 2006. Effect of sericea lespedeza (Lespedeza cuneata) fed as hay, on natural and experimental Haemonchus contortus infections in lambs. Vet. Parasitol. 141 (3-4), 273-278, S0304-4017(06)00346-3 [pii].

MacAdam, J., Griggs, T., 2006. Performance of birdsfoot trefoil, white clover, and other legume-grass mixtures under irrigation in the Intermountain West USA. In: Anonymous PROCEEDINGS OF THE CONFERENCE-NEW ZEALAND GRASSLAND ASSOCIATION. , pp. 355.

Marie-Magdeleine, C., Hoste, H., Mahieu, M., Varo, H., Archimede, H., 2009. In vitro effects of Cucurbita moschata seed extracts on Haemonchus contortus. Vet. Parasitol. 161 (1-2), 99-105, 10.1016/j.vetpar.2008.12.008 [doi].

Marley, C., Cook, R., Keatinge, R., Barrett, J., Lampkin, N., 2003. The effect of birdsfoot trefoil (Lotus corniculatus) and chicory (Cichorium intybus) on parasite intensities and performance of lambs naturally infected with helminth parasites. Vet. Parasitol. 112 (1), 147-155.

Max, R.A., 2010. Effect of repeated wattle tannin drenches on worm burdens, faecal egg counts and egg hatchability during naturally acquired nematode infections in sheep and goats. Vet. Parasitol. 169 (1-2), 138-143, 10.1016/j.vetpar.2009.12.022 [doi]. 
Min, B., Pomroy, W., Hart, S., Sahlu, T., 2004. The effect of short-term consumption of a forage containing condensed tannins on gastro-intestinal nematode parasite infections in grazing wether goats. Small Ruminant Research 51 (3), 279-283.

Molan, A.L., Faraj, A.M., 2010. The effects of condensed tannins extracted from different plant species on egg hatching and larval development of Teladorsagia circumcincta (Nematoda: Trichostrongylidae). Folia. Parasitol. (Praha) 57 (1), $62-68$

Monteiro, M.V.B., Bevilaqua, C.M.L., Morais, S.M., Machado, L.K.A., CamurçaVasconcelos, A.L.F., Campello, C.C., Ribeiro, W.L.C., Mesquita, M.d.A., 2011. Anthelmintic activity of Jatropha curcas L. seeds on Haemonchus contortus. Vet. Parasitol. 182 (2-4), 259-263, http://dx.doi.org/10.1016/j.vetpar.2011.04.010.

Neto, C.C., Krueger, C.G., Lamoureaux, T.L., Kondo, M., Vaisberg, A.J., Hurta, R.A., Curtis, S., Matchett, M.D., Yeung, H., Sweeney, M.I., 2006. MALDI-TOF MS characterization of proanthocyanidins from cranberry fruit (Vaccinium macrocarpon) that inhibit tumor cell growth and matrix metalloproteinase expression in vitro. J. Sci. Food Agric. 86 (1), 18-25.

Niezen, J.H., Waghorn, G.C., Graham, T., Carter, J.L., Leathwick, D.M., 2002. The effect of diet fed to lambs on subsequent development of Trichostrongylus colubriformis larvae in vitro and on pasture. Vet. Parasitol. 105 (4), 269-283, S0304401702000250 [pii]. 
Ollivier, J., Towe, S., Bannert, A., Hai, B., Kastl, E.M., Meyer, A., Su, M.X., Kleineidam, K., Schloter, M., 2011. Nitrogen turnover in soil and global change. FEMS Microbiol. Ecol. 78 (1), 3-16, 10.1111/j.15746941.2011.01165.x [doi].

Ortolani, E.L., Leal, M.L., Minervino, A.H., Aires, A.R., Coop, R.L., Jackson, F., Suttle, N.F., 2013. Effects of parasitism on cellular immune response in sheep experimentally infected with Haemonchus contortus. Vet. Parasitol. 196 (1-2), 230-234, 10.1016/j.vetpar.2013.02.014 [doi].

Paterson, S.M., Casadio, Y.S., Brown, D.H., Shaw, J.A., Chirila, T.V., Baker, M.V., 2013. Laser scanning confocal microscopy versus scanning electron microscopy for characterization of polymer morphology: Sample preparation drastically distorts morphologies of poly (2-hydroxyethyl methacrylate)-based hydrogels. J Appl Polym Sci 127 (6), 4296-4304.

Polashock, J., Zelzion, E., Fajardo, D., Zalapa, J., Georgi, L., Bhattacharya, D., Vorsa, N., 2014. The American cranberry: first insights into the whole genome of a species adapted to bog habitat. BMC Plant. Biol. 14, 165-2229-14-165, 10.1186/1471-2229-14-165 [doi].

Porter, M.L., Krueger, C.G., Wiebe, D.A., Cunningham, D.G., Reed, J.D., 2001. Cranberry proanthocyanidins associate with low-density lipoprotein and inhibit in vitro $\mathrm{Cu} 2$-induced oxidation. J. Sci. Food Agric. 81 (14), 1306-1313.

Puchala, R., Min, B., Goetsch, A., Sahlu, T., 2005. The effect of a condensed tannincontaining forage on methane emission by goats. J. Anim. Sci. 83 (1), 182-186. 
Reed, J.D., 1995. Nutritional toxicology of tannins and related polyphenols in forage legumes. J. Anim. Sci. 73 (5), 1516-1528.

Roberts, C., Beuselinck, P., Ellersieck, M., Davis, D., McGraw, R., 1993. Quantification of Tannis in Birdsfoot Trefoil Germplasm. Crop Sci. 33 (4), 675-679.

Santos, M.C., Xavier, J.K., Amarante, M.R., Bassetto, C.C., Amarante, A.F., 2014. Immune response to Haemonchus contortus and Haemonchus placei in sheep and its role on parasite specificity. Vet. Parasitol. 203 (1-2), 127-138, 10.1016/j.vetpar.2014.02.048 [doi].

Shaik, S.A., Terrill, T.H., Miller, J.E., Kouakou, B., Kannan, G., Kaplan, R.M., Burke, J.M., Mosjidis, J.A., 2006. Sericea lespedeza hay as a natural deworming agent against gastrointestinal nematode infection in goats. Vet. Parasitol. 139 (1-3), 150-157, S0304-4017(06)00142-7 [pii].

Steiner, J., Beuselinck, P., Greene, S., Kamm, J., Kirkbride, J., Roberts, C., 2001. A description and interpretation of the NPGS birdsfoot trefoil core subset collection. Crop Sci. 41 (6), 1968-1980.

Su, X., Howell, A.B., D'Souza, D.H., 2010. The effect of cranberry juice and cranberry proanthocyanidins on the infectivity of human enteric viral surrogates. Food Microbiol. 27 (4), 535-540.

Taiz, L., Zeiger, E., 2006. Plant physiology. 4th. Sinauer Associate, Sunderland, Mass., EUA .

Terrill, T.H., Mosjidis, J.A., Moore, D.A., Shaik, S.A., Miller, J.E., Burke, J.M., Muir, J.P., Wolfe, R., 2007. Effect of pelleting on efficacy of sericea lespedeza hay 
as a natural dewormer in goats. Vet. Parasitol. 146 (1-2), 117-122, S03044017(07)00083-0 [pii].

Terrill, T.H., Waghorn, G.C., Woolley, D.J., McNabb, W.C., Barry, T.N., 1994. Assay and digestion of 14C-labelled condensed tannins in the gastrointestinal tract of sheep. Br. J. Nutr. 72 (3), 467-477.

Terrill, T., Rowan, A., Douglas, G., Barry, T., 1992. Determination of extractable and bound condensed tannin concentrations in forage plants, protein concentrate meals and cereal grains. J. Sci. Food Agric. 58 (3), 321-329.

Vargas-Magana, J.J., Torres-Acosta, J.F., Aguilar-Caballero, A.J., Sandoval-Castro, C.A., Hoste, H., Chan-Perez, J.A., 2014. Anthelmintic activity of acetonewater extracts against Haemonchus contortus eggs: interactions between tannins and other plant secondary compounds. Vet. Parasitol. 206 (3-4), 322327, S0304-4017(14)00535-4 [pii].

USDA. 2015. "Sheep and Lamb Predator and Nonpredator Death Loss in the United States, 2015.” USDA-APHIS-VS-CEAH-NAHMS Fort Collins, CO \#721.0915.

Veríssimo, C.J., Niciura, S.C.M., Alberti, A.L.L., Rodrigues, C.F.C., Barbosa, C.M.P., Chiebao, D.P., Cardoso, D., da Silva, G.S., Pereira, J.R., Margatho, L.F.F., da Costa, R.L.D., Nardon, R.F., Ueno, T.E.H., Curci, V.C.L.M., Molento, M.B., 2012. Multidrug and multispecies resistance in sheep flocks from São Paulo state, Brazil. Vet. Parasitol. 187 (1-2), 209-216, http://dx.doi.org/10.1016/j.vetpar.2012.01.013. 
Vlassoff, A., Leathwick, D.M., Heath, A.C., 2001. The epidemiology of nematode infections of sheep. N. Z. Vet. J. 49 (6), 213-221, 10.1080/00480169.2001.36235 [doi].

von Son-de Fernex, E., Alonso-Diaz, M.A., Valles-de la Mora, B., Capetillo-Leal, C.M., 2012. In vitro anthelmintic activity of five tropical legumes on the exsheathment and motility of Haemonchus contortus infective larvae. Exp. Parasitol. 131 (4), 413-418, 10.1016/j.exppara.2012.05.010 [doi].

Waghorn, G., 1996. Condensed tannins and nutrient absorption from the small intestine. In: Anonymous Animal Science Research and Development, Meeting Future Challenges., pp. 175-193.

Weiss, E.I., Kozlovsky, A., Steinberg, D., Lev-Dor, R., Bar Ness Greenstein, R., Feldman, M., Sharon, N., Ofek, I., 2004. A high molecular mass cranberry constituent reduces mutans streptococci level in saliva and inhibits in vitro adhesion to hydroxyapatite. FEMS Microbiol. Lett. 232 (1), 89-92, 10.1016/S0378-1097(04)00035-7 [doi]. 


\title{
CHAPTER 2
}

In vitro screening of fifty-one birdsfoot trefoil (Lotus corniculatus) strains for anthelmintic efficacy against Haemonchus contortus

by

Carly D. Barone ${ }^{\mathrm{a}}$, Sarah M. Ferguson ${ }^{\mathrm{b}}$, Rebecca N. Brown ${ }^{\mathrm{b}}$, Anne M. Zajac ${ }^{\mathrm{c}}$, Jess D. Reed $^{\mathrm{d}}$, Christian G. Krueger ${ }^{\mathrm{d}}$, Katherine H. Petersson ${ }^{\mathrm{a}}$

is prepared for submission to the journal Veterinary Parasitology

\begin{abstract}
Affiliations:
${ }^{a}$ Department of Fisheries, Animal, and Veterinary Sciences, University of Rhode Island, 120 Flagg Road, CBLS Rm 177, Kingston, Rhode Island, 02881, USA.

${ }^{\mathrm{b}}$ Department of Plant Sciences and Entomology, University of Rhode Island, $9 \mathrm{E}$ Alumni Ave, Woodward Hall Rm 236, Kingston, RI, 02881, USA.

${ }^{c}$ Biomedical Sciences and Pathobiology, VA-MD Regional College of Veterinary Medicine, Virginia Tech, Center for Molecular Medicine and Infectious Diseases, 1410 Prices Fork Road, Blacksburg, VA, 24061 USA.

${ }^{\mathrm{d}}$ Animal Science, University of Wisconsin, Madison, 1675 Observatory Drive, Rm 1146, Madison, Wisconsin, 53706, USA.
\end{abstract}




\begin{abstract}
Faced with severe and global anthelmintic resistance, the search for alternative methods of gastrointestinal nematode (GIN) control in small ruminants is imperative. Investigation into this problem has revealed that some forages containing condensed tannins (CT), also called proanthocyanidins (PAC), suppress GIN infection. The antiparasitic activity of purified proanthocyanidin extract (Bruce-PAC) and crude aqueous extracts (BFT-AqE) from 51 strains of BFT were tested using the following in vitro assays: 1) egg hatching and viability of L1 Haemonchus contortus larvae and 2) exsheathment and viability of L3 H. contortus larvae. The BFT-AqE was prepared by soaking BFT powder in water at room temperature for $24 \mathrm{~h}$, and then the plant matter was removed, leaving an aqueous extract. 1) For in vitro egg hatch inhibition and mortality of L1 larvae, $H$. contortus eggs were exposed to varying concentrations of BFT extracts for 24 h. 2) For in vitro exsheathment, 2,000 H. contortus L3 larvae were exposed to BFT extracts prior to exsheathment. Proanthocyanidin content ranged between 1.4 and $63.8 \mathrm{mg} \mathrm{PAC} / \mathrm{g}$ powder across $51 \mathrm{BFT}$ strains. Inhibition of egg hatch and L1 mortality were observed: at $3 \mathrm{mg} / \mathrm{mL}$ BFT-AqE, percent inhibition of egg hatch and L1 mortality ranged between 0 and 100\% across 51 strains tested. Inhibition of exsheathment and L3 mortality was observed across thirteen tested strains: at $25 \mathrm{mg} / \mathrm{mL}$, percent inhibition of exsheathment bridged between 0 and $75 \%$ inhibition. Incubation in Bruce-PAC exhibited 8\% egg hatch inhibition, 98\% L1 mortality, 4\% percent L3 mortality, and 100\% exsheathment inhibition at the highest concentration of $6 \mathrm{mg} / \mathrm{mL}$ Bruce-PAC. Results indicate that strains of BFT-AqE inhibited egg hatch and inhibited larval mortality, but the degree of inhibition varied.
\end{abstract}


Results also indicate that Bruce-PAC did not inhibit egg hatch or mortality of L3 larvae, but did inhibit mortality of L1 larvae and inhibit exsheathment. When comparing PAC content of varietal BFT powders to anthelmintic efficacy of aqueous extracts, there were no significant correlations. Since PAC content was not related to activity, PAC structure was investigated for its potential influence on bioactivity. Further testing of PAC extracted from different varieties will provide additional information about the anthelmintic efficacy of strains of BFT for small ruminant GIN control.

\section{Introduction}

Gastrointestinal nematodes (GIN) are one of the most pressing health concerns faced by small ruminant producers. The barber pole worm (Haemonchus contortus) is the most pathogenic of the small ruminant GIN, responsible for the majority of economic losses in sheep production worldwide (Veríssimo et al., 2012). An infected animal sheds eggs onto pasture through defecation, which hatch into first stage (L1), develop to second (L2) and third stage (L3) larvae. Infective L3 larvae migrate out of the feces and are ingested by a grazing animal. In the rumen, L3 larvae undergo exsheathment and then develop into L4 and eventually adult worms that reside in the abomasum, where they feed on the blood of the host, mate, and produce eggs. The

prevalence of resistance to commercial dewormers in GIN has created the need for alternative methods of control. Plants rich in condensed tannins (CT), also called proanthocyanidins (PAC), and other secondary compounds have been explored for their anthelmintic efficacy, though the mechanism of action is unknown. Proanthocyanidins, naturally occurring secondary compounds in the stems and leaves 
of plants (Reed, 1995), have a complex structure that can vary depending on the plant species and variety. Characteristic variations include hydroxylations, glysosylations, and A- and B- type linkages, all of which have potential to influence their biological activity.

Sericea lespedeza (Lespedeza cuneata), a tropical perennial, has been shown to be effective in managing GIN infections (Lange et al., 2006; Shaik et al., 2006) however, as with other plants investigated for their anthelmintic efficacy (Assis et al., 2003; Marie-Magdeleine et al., 2009), it is not adapted to the New England climate. Birdsfoot trefoil (BFT, Lotus corniculatus), a drought resistant legume (Hedqvist et al., 2000) that tolerates low soil fertility and is adapted to the northeastern United States, has exhibited anthelmintic efficacy against $H$. contortus in vivo by reducing total worm burden (Heckendorn et al., 2007). Grazing this legume provides additional benefits to the animal by minimizing bloat and improving protein uptake (Min et al., 2006) and may provide a feasible option for producers to use as hay or forage to manage small ruminant GIN.

There are numerous varieties of BFT that differ in biochemical, morphological, and agronomic characteristics (Steiner et al., 2001). Fourteen BFT varieties investigated for PAC concentrations were found to differ substantially, ranging from 14 to $32 \mathrm{mg} / \mathrm{g}$ dry matter (Grabber et al., 2014). In a companion paper to this study, Reed et al. (in preparation) reported PAC concentrations ranging from 1.4 to $63.8 \mathrm{mg}$ $\mathrm{PAC} / \mathrm{g}$ powder for the $51 \mathrm{BFT}$ strains. North American BFT cultivars, such as Norcen, are generally low in PAC content (Steiner et al., 2001; Grabber et al., 2014), and lower PAC content is correlated with a reduction in the associated bitterness 
(Monagas et al., 2010). It is essential for a small ruminant to readily consume a PAC containing forage, therefore a reduction in bitterness, would ultimately increase palatability. If PAC content is positively correlated with anthelmintic activity then the North American cultivars with lower PAC content, but higher palatability, may have less anti-parasitic activity.

This study utilizes in vitro methods to investigate the anthelmintic efficacy of fifty-one strains of BFT and to determine correlation between PAC structure observed by Reed et al. (in preparation) and anti-parasitic activity. The specific objectives of this study are to: 1) test the anthelmintic efficacy of fifty-one BFT varieties against $H$. contortus egg hatching and L1 survival; 2) test the effects of the top performing BFT varieties (13) on $H$. contortus L3 survival and exsheathment; and 3) determine if the PAC content and structure of a specific strain of BFT can be correlated to the biological activity of that strain.

\section{Materials and Methods}

\subsection{Birdsfoot trefoil}

\subsubsection{Harvest}

Birdsfoot trefoil strains (51) were grown from seed in a greenhouse (University of Rhode Island, Kingston, RI, USA), under mist, in late January 2012 and hardened off outside in early May before being transplanted to field plots. Accessions were originally chosen based on previously reported PAC content, ranging between 2 and 105 mg/g dry matter (Roberts et al., 1993; Grabber et al., 2014; GRIN database) and seed for each strain was acquired (Table 1). Once transplanted and established in field plots, plant matter was harvested (clipped and bagged), pre-bloom and kept at - 
$20^{\circ} \mathrm{C}$ until the frozen tissue samples were freeze-dried (Labconco Bulk Tray Dryer 115V and FreeZone 6 Liter Benchtop Freeze Dry System, Labconco Corporation, Kansas City, MO, USA.) and ground into fine powder.

Table 1: Seed suppliers of the 51 BFT strains used in the anthelmintic screening tests.

\begin{tabular}{cc}
\hline BFT & Seed provided by: \\
Strain & \\
\hline Bull & Welter Seed \& Honey Co. (Onslow, Iowa, USA) \\
Norcen & Welter Seed \& Honey Co. (Onslow, Iowa, USA) \\
Bruce & Welter Seed \& Honey Co. (Onslow, Iowa, USA) \\
Empire & Ernst Conservation Seeds (Meadville, PA, USA) \\
Leo & Ernst Conservation Seeds (Meadville, PA, USA) \\
Pardee & Seedway (Vermont Warehouse, Shoreham, VT, USA) \\
B1230 & Cornell University (Ithaca, NY, USA) \\
B0730 & Cornell University (Ithaca, NY, USA) \\
S3149 & Margot Forde Germplasm Centre (New Zealand) \\
3428 & University of Minnesota (Minneapolis, MN, USA) \\
$162456-655626^{1}$ & USDA National Plant Germplasm Repository (NPGS, \\
& Pullman, WA, USA) \\
\hline
\end{tabular}

${ }^{1}$ Includes the 41 BFT accessions with 6-digit numbers.

\subsubsection{Birdsfoot trefoil crude aqueous extract (BFT-AqE)}

Ground BFT powder $(360 \mathrm{mg})$ was placed in a Falcon ${ }^{\mathrm{TM}}$ tube and room temperature tap water was added up to a volume of $30 \mathrm{~mL}(12 \mathrm{mg}$ powder $/ \mathrm{mL}$; used in egg hatch and L1 mortality assay). A $50 \mathrm{mg}$ powder/mL extract was prepared in the same way for the L3 mortality and exsheathment inhibition assay. Plant matter was 
mixed with the water, set in the dark for 24 hours, and then centrifuged ( $200 \mathrm{x} \mathrm{g}) 5$ minutes and the supernatant removed and used as aqueous extract (AqE). Although extracted supernatant was not $30 \mathrm{~mL}$ in total, the concentrations used to describe the BFT-AqE extracts were of the original $12 \mathrm{mg}$ powder/mL stock solution. The stock solutions of aqueous extracts, from which serial dilutions were made, were used the same day in the in vitro assays. The stock solutions were stored at $-20^{\circ} \mathrm{C}$ until phytochemical analysis.

\subsection{Phytochemical analyses}

\subsubsection{Concentration of PAC by DMAC}

The concentration of PAC in the ground BFT powders was determined using the DMAC (4-(dimethylamino)cinnamaldehyde) method by the Reed Research Group at the University of Wisconsin-Madison, Madison, Wisconsin, USA. Concentrations of PAC in the BFT powders are not expected to be similar of the aqueous extracts. Proanthocyanidin extract was isolated from the BFT powders according to the established method for PAC isolation (Krueger et al., 2016; Feliciano et al., 2012a). Polystyrene 96-well plates and a plate reader were used to measure the absorbance of each DMAC assay. A BFT ('Bruce' strain) PAC standard was used to reflect the structural heterogeneity of BFT PAC. Calibration standards, blanks ( $80 \%$ ethanol), and samples $(70 \mu \mathrm{l})$ were dispensed into wells. Absorbance of each well was read at $640 \mathrm{~nm}$ at 30 -second intervals for one hour, totaling 121 readings per well. The corresponding blank absorbance was subtracted for standards and samples. 


\subsubsection{Birdsfoot trefoil 'Bruce' proanthocyanidin extract (Bruce-PAC)}

The 'Bruce' variety of BFT was used as the standard for the DMAC assay and was the only variety of BFT that proanthocyanidin was extracted from.

Proanthocyanidin was isolated from the birdsfoot trefoil ground powder using solidphase chromatography, according to a well-established method for PAC isolation (Howell et al., 2005). Reverse phase (C18) followed by adsorption chromatography (Sephadex LH-20) was used to extract and isolate the total PAC. Isolated PAC was stored at room temperature in the dark and re-suspended in water when used for in vitro assays.

\subsubsection{Structure by MALDI-TOF MS}

The Reed Research Group at the University of Wisconsin-Madison, Madison, WI, USA spectrally analyzed the BFT-AqE extracts according to previously established methods (Krueger et al., 2013; Feliciano et al., 2012b). All samples were subjected to PAC enrichment by passing the extracts through a Sephdex LH-20 column prior to MALDI-TOF MS. Isolated PAC samples were then combined with the matrix (2,5-dihydroxybenzoic acid) and analyzed in a Bruker BIFLEX III MALDI-TOF Mass Spectrometer (Billerica, MA), equipped with Bruker ULTRAFLEX $^{\circledR}$ III MALDI TOF/TOF mass spectrometer (Billerica, MA, USA), equipped with delayed extraction and a SmartBeam ${ }^{\circledR}$ laser. Mass spectra were calibrated with bradykinin and glucagon as external standards. All PAC samples were analyzed using linear and reflectron detection in positive ion mode. MALDI-TOF mass spectra of the isolated PAC were interpreted by predictive equations applied to 
determine range and degree of polymerization. Results of structural analyses have been reported in a companion publication (Reed et al., in preparation).

\subsection{In vitro assays}

\subsubsection{Study subjects}

This study was conducted with the approval of the Institutional Animal Care and Use Committee (IACUC) of the University of Rhode Island. The donor animals used for this study were Dorset/Hampshire crossbred lambs born and housed at the University of Rhode Island Peckham Farm, located in Kingston, RI, USA. Lambs were brought indoors and remained in indoor housing for the entire study. Lambs were dewormed orally with two anthelmintics; levamisole hydrochloride $(8.8 \mathrm{mg} / \mathrm{kg}$ BW, Levasole ${ }^{\circledR}$, Schering-Plough Animal Health Corp., Union, NJ) and ivermectin $\left(0.2 \mathrm{mg} / \mathrm{kg} \mathrm{BW}\right.$, Ivomec ${ }^{\circledR}$, Merial Inc., Duluth, GA) and experimentally infected with 10,000 L3 H. contortus provided by the Zajac parasitology laboratory at the VirginiaMaryland College of Veterinary Medicine, Virginia Tech, Blacksburg, VA, USA.

Once eggs were present in the fecal samples (approximately 35 days), feces were used for the collection of H. contortus eggs for use in the egg hatch assays.

\subsubsection{Egg hatch and L1 mortality assay}

The anthelmintic efficacy of BFT-AqE and Bruce-PAC on hatching of $H$. contortus eggs was determined by modification of previously published procedures (Assis et al., 2003; Marie-Magdeleine et al., 2009). Fresh feces were collected directly from the donor animal's rectum. Tap water was added to the feces to create a slurry by mashing the fecal pellets in the water and the suspension was washed over sieves $(1000,355,150,38$, and $25 \mu \mathrm{m})$. Eggs retained on the last two sieves were 
recovered using flotation with a concentrated salt solution (Fecasol®, Vetoquinol U.S.A., Inc., Fort Worth, TX, USA) and collected on glass cover slips. Collected eggs were rinsed off the glass cover slips with tap water and kept in a $15 \mathrm{~mL}$ Falcon ${ }^{\mathrm{TM}}$ tube at $4{ }^{\circ} \mathrm{C}$ to prevent hatching. Eggs were harvested daily and retained at $4{ }^{\circ} \mathrm{C}$ for $1-2$ hours prior to being used in each assay.

Eggs were placed (100 eggs/well in $100 \mu \mathrm{H}_{2} \mathrm{O}$ ) into 24-well flat-bottomed microtitre plates (Corning ${ }^{\mathrm{TM}}$, Falcon ${ }^{\mathrm{TM}}$, Polystyrene Microplates, Corning Life Sciences, Tewksbury, MA, USA). Stock solutions in water of BFT-AqE (12 mg powder $/ \mathrm{mL})$ and Bruce-PAC (12 mg PAC/mL), prepared fresh prior to each assay, were serially diluted with water and added in equal volume to the wells. Thiabendazole (Thiabendazole (TBZ), Thermo Fisher Scientific Inc., Waltham, MA, USA), a commercial dewormer, in dimethyl sulfoxide $(0.5 \mu \mathrm{g} / \mathrm{mL})$ (Dimethyl Sulfoxide (DMSO), Fisher BioReagents ${ }^{\mathrm{TM}}$, Thermo Fisher Scientific Inc., Waltham, MA, USA), was used as a positive control to inhibit egg hatch. Water was used as a negative control for egg hatching and larval viability. Water (890 $\mu$ l), DMSO (10 $\mu 1)$, and serial concentrations of BFT-AqE, Bruce-PAC, or water were added $(1 \mathrm{~mL})$ to each well for a total volume of $2 \mathrm{~mL}$. Final concentrations in wells for BFT-AqE were $6,3,1.5$, and $0.75 \mathrm{mg}$ powder $/ \mathrm{mL}$ and final concentrations in wells for Bruce-PAC were $6,3,1.5$, and $0.75 \mathrm{mg} \mathrm{PAC} / \mathrm{mL}$. Five replicates were run for each set of dilutions and controls. Eggs were incubated at $26^{\circ} \mathrm{C}$ for 24 hours and determined to be hatched or not hatched, and results were expressed as percent egg hatch inhibition. To determine the anthelmintic efficacy of BFT-AqE and Bruce-PAC on L1 larvae, the hatched L1 were quantified and determined motile (live) or non-motile (dead), based 
on observations for five seconds (Skantar et al., 2005; Katiki et al., 2013). Results were expressed as percent mortality of hatched larvae. Percentages were adjusted to the negative control (water, $0.0 \mathrm{mg} / \mathrm{mL}$ ) values for each individual assay, determined by using the following formula: mortality or inhibition $(\%)=(\mathrm{A}-\mathrm{B}) /(\mathrm{A}) \times 100$ (Acharya et al., 2014). Where $\mathrm{A}=\%$ viable or hatched in the water control and $\mathrm{B}=\%$ viable or hatched in the different concentrations of extracts.

\subsubsection{L3 mortality and exsheathment inhibition assay}

The L3 H. contortus larvae used in this assay were provided by the Zajac parasitology laboratory at the Virginia-Maryland College of Veterinary Medicine, Virginia Tech, Blacksburg, VA, USA. Larvae were kept at $4^{\circ} \mathrm{C}$ until use in the L3 mortality and exsheathment inhibition assay and were no older than 3 months of age when used. Larvae were observed for five seconds and classifyed as motile (alive) or non-motile (dead). If no movement was observed for 5 seconds, larvae were considered non-motile (dead) (Skantar et al., 2005; Katiki et al., 2013).

Based upon the results in the egg hatch and L1 viability assays as well as agronomic performance (Ferguson, in preparation; thesis), the top thirteen BFT varieties were selected to investigate the effect of BFT-AqE and Bruce-PAC on L3 larvae and exsheathment. Stock solutions of BFT-AqE (50 mg powder/mL) and Bruce-PAC (12 mg/mL), made fresh for each assay, were serially diluted using tap water. The larvae were incubated in BFT-AqE (25 mg powder/mL), Bruce-PAC (6, 3, 1.5, and $0.75 \mathrm{mg} \mathrm{PAC} / \mathrm{mL}$ ), or a water control for 24 hours at $37^{\circ} \mathrm{C}$. The sheathed $\mathrm{L} 3$ $(2,000)$ were added to Earle's Balanced Salt Solution (EBSS, Sigma-Aldrich ${ }^{\circledR}$, Inc., Natick, MA, USA) up to a volume of $1 \mathrm{~mL}$ in a $15 \mathrm{~mL} \mathrm{Falcon}^{\mathrm{TM}}$ tube. Water control 
$(1 \mathrm{~mL})$ or treatment $(1 \mathrm{~mL})$ was added for a total volume of $2 \mathrm{~mL}$. After 24 hours, percent viability was determined. Exsheathment was induced following a modification of Conder and Johnson (1996). Tubes were covered with Parafilm M® (Parafilm M®, Bemis Company, Inc., Neenah, WI). A glass pipet tip, connected to $\mathrm{CO}_{2}$, was pushed through the Parafilm $\mathrm{M}{ }^{\circledR}$ and positioned in the EBSS larval solution. Larvae were rapidly bubbled with $\mathrm{CO}_{2}$ for 15 minutes, capped tightly, and placed back into the incubator for 18 hours at $37^{\circ} \mathrm{C}$. After the 18 -hour exsheathment incubation, 100 larvae/tube were determined dead or alive, and live larvae were determined sheathed or exsheathed. Results were expressed as percent mortality of all larvae and percent exsheathment inhibition of the live larvae. Percentages were adjusted to the negative control (water, $0.0 \mathrm{mg} / \mathrm{mL}$ ) values for each individual assay, determined by using the following formula: mortality or inhibition $(\%)=(\mathrm{A}-\mathrm{B}) /(\mathrm{A}) \mathrm{x} 100$ (Acharya et al., 2014). Where $\mathrm{A}=\%$ viable or exsheathed in the water control and $\mathrm{B}=\%$ viable or exsheathed in the different concentrations of extracts.

\subsection{Statistical analysis}

Egg hatch, L1 and L3 mortality, and exsheathment inhibition data were analyzed statistically using an analysis of variance (ANOVA) and means separated with Dunnett's $t$ test using the GLM Procedure in SAS (SAS Institute Inc., Cary, NC). Treatment means were compared using a least significant difference (LSD) value with significance defined as $p \leq 0.05$. The concentration of extracts required to prevent $50 \%$ of egg hatching $\left(\mathrm{LC}_{50}\right)$ and $90 \%$ of egg hatching $\left(\mathrm{LC}_{90}\right)$ were calculated using PROBIT procedure in SAS (SAS Institute Inc., Cary, NC). The CORR procedure in 
SAS was used to test correlations between the structural characteristics of the BFT powders and the anthelmintic efficacy of the corresponding aqueous extracts.

\section{Results}

\subsection{Egg hatch and L1 mortality assay}

All 51 strains of BFT-AqE at $6 \mathrm{mg}$ powder $/ \mathrm{mL}$ exhibited significant egg hatch inhibition when compared to the negative control $(0.0 \mathrm{mg} / \mathrm{mL} ; p \leq 0.001)$. Out of the 51 strains, 40 of them proved significant egg hatch inhibition at $3 \mathrm{mg} / \mathrm{mL}$ BFT-AqE compared to the negative control, but only 12 strains had significant egg hatch inhibition in $1.5 \mathrm{mg} / \mathrm{mL}$ and 3 strains in $0.75 \mathrm{mg} / \mathrm{mL}$ (Table 2). Of the 51 strains of BFT that were tested, eleven of them exhibited $100 \%$ egg hatch inhibition at concentrations of 3 and $6 \mathrm{mg}$ powder $/ \mathrm{mL}$ BFT-AqE and $70 \%$ of the strains proved to be $>90 \%$ efficacious against egg hatching at $6 \mathrm{mg} / \mathrm{mL}$ (Table 2).

The percent of egg hatch inhibition averages (mean \pm SEM) of the 51 BFT strains in $6.0,3.0,1.5$, and $0.75 \mathrm{mg}$ powder/mL BFT-AqE were $89 \pm 3,59 \pm 5,17 \pm 4$, and $4 \pm 2$ respectively (Fig. 1) (ranging between $0 \%$ and $100 \%$ inhibition in 3 and 1.5 $\mathrm{mg} / \mathrm{mL}$, Table 2). Of the eggs that hatched, the percent of larval mortality averages $(\mathrm{mean} \pm \mathrm{SEM})$ in $6.0,3.0,1.5$, and $0.75 \mathrm{mg}$ powder/mL BFT-AqE were $36 \pm 6,21 \pm$

4, $14 \pm 3$, and $5 \pm 1$ respectively (Fig. 1) (ranging between $0 \%$ to $100 \%$ in 6.0 and 3.0 $\mathrm{mg}$ powder $/ \mathrm{mL}$ BFT-AqE, and ranging from $0 \%$ to $81 \%$ in $1.5 \mathrm{mg}$ powder $/ \mathrm{mL}$ BFTAqE, Table 2). Water control $(0.0 \mathrm{mg} / \mathrm{mL})$ values for each individual assay, averaged $12 \pm 0$ percent egg hatch inhibition and $1 \pm 0$ percent L1 mortality (mean \pm SEM). The only significant difference found between the 12 water controls was one average 
percent egg hatch inhibition differed compared to 4 of the control groups $(p \leq 0.05)$. Average percent egg hatch inhibition in $\mathrm{TBZ}$ was $97 \pm 0$ (mean $\pm \mathrm{SEM})$.

The $\mathrm{LC}_{50}$ against egg hatching of the $51 \mathrm{BFT}$ strains ranged from 0.66 to 9.36 $\mathrm{mg} / \mathrm{mL}$, averaging $2.8 \pm 0.3 \mathrm{mg} / \mathrm{mL}$ (mean \pm SEM) (Table 3). When comparing the egg hatch inhibition percentages to the $\mathrm{L} 1$ mortality percentages, at $1.5 \mathrm{mg}$ powder $/ \mathrm{mL}$, there was a significant correlation between $\mathrm{L} 1$ mortality and egg hatch inhibition $\left(R^{2}=0.78\right)$ (Fig. 2. C). The Bruce-PAC that was screened, exhibited $8 \%$ egg hatch inhibition (compared to $89 \%$ egg hatch inhibition using Bruce AqE) and $98 \% \mathrm{~L} 1$ mortality at the highest concentration of $6 \mathrm{mg} \mathrm{PAC} / \mathrm{mL}$ (Table 2). The $\mathrm{LC}_{50}$ of Bruce-PAC against egg hatching was $1795 \mathrm{mg} / \mathrm{mL}$ (Table 3).

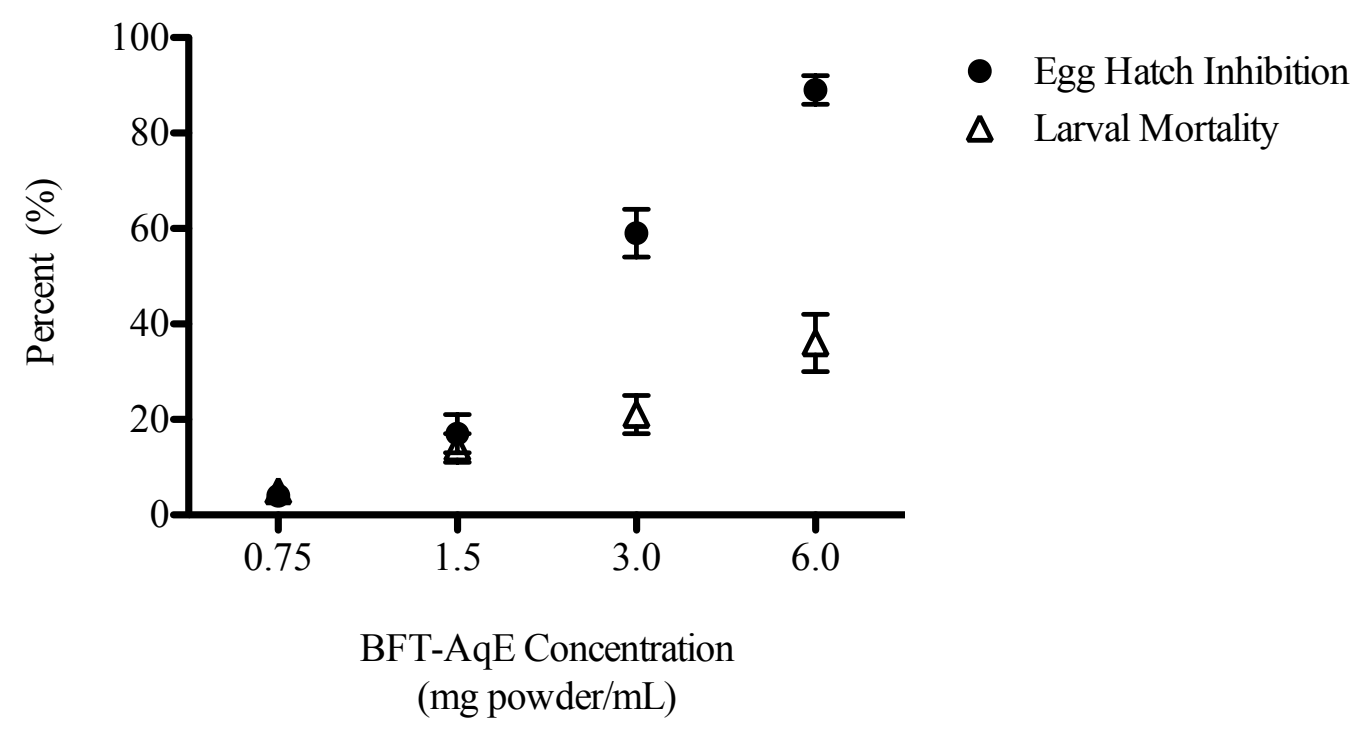

Figure 1. The percent of egg hatch inhibition averages (mean \pm SEM) and percent of larval mortality averages (mean $\pm \mathrm{SEM}$ ) in $6.0,3.0,1.5$, and $0.75 \mathrm{mg}$ powder $/ \mathrm{mL}$ BFT-AqE. 
Table 2: Effect of aqueous extracts from 51 strains of BFT and purified Bruce-PAC extract on Haemonchus contortus egg hatch and larval mortality post-hatch.

\begin{tabular}{|c|c|c|c|c|c|c|c|c|c|}
\hline \multirow{3}{*}{$\begin{array}{l}\text { BFT } \\
\text { Strain }\end{array}$} & \multirow{3}{*}{$\begin{array}{c}\text { PAC } \\
\text { (DMAC) } \\
\mathrm{mg} / \mathrm{g}\end{array}$} & \multicolumn{4}{|c|}{ Egg Hatch Inhibition (\%) } & \multicolumn{4}{|c|}{ L1 Mortality (\%) } \\
\hline & & \multicolumn{4}{|c|}{$\mathrm{mg} / \mathrm{mL}$} & \multicolumn{4}{|c|}{$\mathrm{mg} / \mathrm{mL}$} \\
\hline & & 0.75 & 1.5 & 3 & 6 & 0.75 & 1.5 & 3 & 6 \\
\hline $\mathrm{Bull}^{++}$ & 3.7 & $4 \pm 1$ & $90 \pm 12^{*}$ & $100 \pm 0 *$ & $100 \pm 0 *$ & $1 \pm 1$ & $58 \pm 22$ & -- & -- \\
\hline Empire $^{++}$ & 4.2 & $4 \pm 2$ & $87 \pm 12^{*}$ & $100 \pm 0^{*}$ & $100 \pm 0 *$ & $3 \pm 1$ & $66 \pm 14$ & -- & -- \\
\hline Norcen $^{++}$ & 3.5 & $17 \pm 3^{*}$ & $78 \pm 1^{*}$ & $100 \pm 0 *$ & $100 \pm 0 *$ & $59 \pm 7 *$ & $81 \pm 4^{*}$ & -- & -- \\
\hline $\mathrm{Leo}^{++}$ & 6.0 & $11 \pm 2$ & $48 \pm 10^{*}$ & $100 \pm 0^{*}$ & $100 \pm 0 *$ & $2 \pm 1$ & $37 \pm 4^{*}$ & -- & -- \\
\hline Pardee $^{++}$ & 8.1 & $2 \pm 1$ & $30 \pm 3 *$ & $100 \pm 0 *$ & $100 \pm 0 *$ & $19 \pm 3 *$ & $47 \pm 5^{*}$ & -- & -- \\
\hline Bruce $^{++}$ & 12.0 & $0 \pm 2$ & $6 \pm 2$ & $70 \pm 10^{*}$ & $89 \pm 6^{*}$ & $1 \pm 1$ & $9 \pm 4$ & $36 \pm 9^{*}$ & $84 \pm 7 *$ \\
\hline Bruce-PAC & Standard & $2 \pm 2$ & $6 \pm 3$ & $13 \pm 2$ & $8 \pm 3$ & $70 \pm 2 *$ & $95 \pm 1 *$ & $94 \pm 1 *$ & $98 \pm 1 *$ \\
\hline $325379^{++}$ & 1.5 & $99 \pm 1 *$ & $100 \pm 0^{*}$ & $100 \pm 0^{*}$ & $100 \pm 0 *$ & $0 \pm 0$ & -- & -- & -- \\
\hline $228286^{++}$ & 3.7 & $9 \pm 5$ & $53 \pm 14^{*}$ & $100 \pm 0 *$ & $100 \pm 0 *$ & $7 \pm 2$ & $20 \pm 21$ & -- & -- \\
\hline
\end{tabular}




\begin{tabular}{|c|c|c|c|c|c|c|c|c|c|}
\hline \multirow{3}{*}{$\begin{array}{c}\text { BFT } \\
\text { Strain }\end{array}$} & \multirow{3}{*}{$\begin{array}{c}\text { PAC } \\
\text { (DMAC) } \\
\mathrm{mg} / \mathrm{g}\end{array}$} & \multicolumn{4}{|c|}{ Egg Hatch Inhibition (\%) } & \multicolumn{4}{|c|}{ L1 Mortality (\%) } \\
\hline & & & & & \multirow[b]{2}{*}{6} & \multicolumn{4}{|c|}{$\mathrm{mg} / \mathrm{mL}$} \\
\hline & & 0.75 & 1.5 & 3 & & 0.75 & 1.5 & 3 & 6 \\
\hline $162456^{++}$ & 4.2 & $0 \pm 2$ & $17 \pm 17$ & $100 \pm 0 *$ & $100 \pm 0 *$ & $1 \pm 1$ & $1 \pm 1$ & -- & -- \\
\hline $193725^{++}$ & 24.4 & $0 \pm 2$ & $11 \pm 4$ & $100 \pm 0 *$ & $100 \pm 0 *$ & $0 \pm 1$ & $0 \pm 1$ & -- & -- \\
\hline $246730^{++}$ & 43.2 & $2 \pm 2$ & $4 \pm 3$ & $100 \pm 0 *$ & $100 \pm 0 *$ & $3 \pm 1$ & $6 \pm 2^{*}$ & -- & -- \\
\hline $180171^{++}$ & 21.1 & $3 \pm 2$ & $9 \pm 1^{*}$ & $100 \pm 0 *$ & $100 \pm 0 *$ & $4 \pm 1$ & $7 \pm 1$ & -- & -- \\
\hline $383689^{++}$ & 2.8 & $6 \pm 1$ & $12 \pm 2$ & $84 \pm 4 *$ & $100 \pm 0 *$ & $1 \pm 0$ & $7 \pm 2$ & $27 \pm 8^{*}$ & -- \\
\hline $255304^{++}$ & -- & $4 \pm 1$ & $2 \pm 3$ & $84 \pm 8^{*}$ & $100 \pm 0 *$ & $8 \pm 1$ & $16 \pm 2$ & $17 \pm 6$ & -- \\
\hline $227315^{++}$ & 19.0 & $0 \pm 2$ & $0 \pm 3$ & $74 \pm 8^{*}$ & $100 \pm 0 *$ & $4 \pm 1$ & $3 \pm 1$ & $69 \pm 17$ & -- \\
\hline $226796^{++}$ & 9.0 & $3 \pm 1$ & $2 \pm 1$ & $70 \pm 4 *$ & $100 \pm 0 *$ & $6 \pm 1$ & $13 \pm 4$ & $16 \pm 6$ & -- \\
\hline $258446^{++}$ & 16.8 & $3 \pm 4$ & $4 \pm 2$ & $67 \pm 2 *$ & $100 \pm 0 *$ & $0 \pm 0$ & $5 \pm 1$ & $10 \pm 3 *$ & -- \\
\hline $383687^{++}$ & 1.4 & $1 \pm 2$ & $4 \pm 2$ & $63 \pm 3^{*}$ & $100 \pm 0 *$ & $9 \pm 2$ & $10 \pm 2$ & $32 \pm 5^{*}$ & -- \\
\hline $214110^{++}$ & 6.1 & $0 \pm 2$ & $3 \pm 1$ & $44 \pm 5^{*}$ & $100 \pm 0 *$ & $0 \pm 0$ & $0 \pm 0$ & $5 \pm 2$ & -- \\
\hline
\end{tabular}




\begin{tabular}{|c|c|c|c|c|c|c|c|c|c|}
\hline \multirow{3}{*}{$\begin{array}{c}\text { BFT } \\
\text { Strain }\end{array}$} & PAC & \multicolumn{4}{|c|}{ Egg Hatch Inhibition (\%) } & \multicolumn{4}{|c|}{ L1 Mortality (\%) } \\
\hline & \multirow{2}{*}{$\begin{array}{c}\text { (DMAC) } \\
\mathrm{mg} / \mathrm{g}\end{array}$} & \multicolumn{4}{|c|}{$\mathrm{mg} / \mathrm{mL}$} & \multicolumn{4}{|c|}{$\mathrm{mg} / \mathrm{mL}$} \\
\hline & & 0.75 & 1.5 & 3 & 6 & 0.75 & 1.5 & 3 & 6 \\
\hline $234670^{++}$ & 7.1 & $5 \pm 1$ & $6 \pm 2$ & $44 \pm 5^{*}$ & $100 \pm 0 *$ & $3 \pm 1$ & $6 \pm 2$ & $8 \pm 2^{*}$ & -- \\
\hline $\mathrm{B} 0730^{++}$ & 12.3 & $5 \pm 1$ & $2 \pm 1$ & $38 \pm 4^{*}$ & $100 \pm 0 *$ & $3 \pm 1$ & $2 \pm 1$ & $9 \pm 4$ & -- \\
\hline $306182^{++}$ & 2.8 & $11 \pm 3$ & $10 \pm 2$ & $34 \pm 4^{*}$ & $100 \pm 0 *$ & $1 \pm 1$ & $3 \pm 1$ & $9 \pm 3$ & -- \\
\hline 310843 & 15.3 & $0 \pm 2$ & $0 \pm 1$ & $13 \pm 5$ & $100 \pm 0^{*}$ & $2 \pm 9$ & $7 \pm 2$ & $15 \pm 4$ & -- \\
\hline $384882^{++}$ & -- & $4 \pm 1$ & $6 \pm 2$ & $61 \pm 4^{*}$ & $100 \pm 0 *$ & $3 \pm 1$ & $3 \pm 1$ & $14 \pm 4^{*}$ & -- \\
\hline $194228^{++}$ & 60.2 & $0 \pm 2$ & $0 \pm 3$ & $100 \pm 0 *$ & $100 \pm 0^{*}$ & $13 \pm 3$ & $16 \pm 4$ & -- & -- \\
\hline $304523^{++}$ & 17.2 & $11 \pm 4$ & $54 \pm 5^{*}$ & $99 \pm 1^{*}$ & $99 \pm 0^{*}$ & $9 \pm 2$ & $33 \pm 3$ & $100 \pm 0^{+}$ & $100 \pm 0^{+}$ \\
\hline $655626^{++}$ & 4.2 & $2 \pm 3$ & $19 \pm 1$ & $84 \pm 8^{*}$ & $99 \pm 1^{*}$ & $26 \pm 6$ & $33 \pm 5$ & $64 \pm 5^{*}$ & $100 \pm 0^{+}$ \\
\hline $251143^{++}$ & 5.2 & $16 \pm 2 *$ & $44 \pm 23^{*}$ & $78 \pm 2 *$ & $99 \pm 1^{*}$ & $1 \pm 0$ & $12 \pm 1$ & $44 \pm 3$ & $100 \pm 0^{+}$ \\
\hline $262530^{++}$ & 11.4 & $0 \pm 2$ & $10 \pm 3$ & $70 \pm 10^{*}$ & $99 \pm 1^{*}$ & $1 \pm 1$ & $4 \pm 1$ & $20 \pm 13$ & $19 \pm 4$ \\
\hline $\mathrm{S} 3149^{++}$ & 46.4 & $3 \pm 1$ & $0 \pm 1$ & $42 \pm 7^{*}$ & $98 \pm 1^{*}$ & $0 \pm 0$ & $1 \pm 1$ & $7 \pm 2$ & $44 \pm 9$ \\
\hline
\end{tabular}




\begin{tabular}{|c|c|c|c|c|c|c|c|c|c|}
\hline \multirow{3}{*}{$\begin{array}{c}\text { BFT } \\
\text { Strain }\end{array}$} & \multirow{3}{*}{$\begin{array}{c}\text { PAC } \\
\text { (DMAC) } \\
\mathrm{mg} / \mathrm{g}\end{array}$} & \multicolumn{4}{|c|}{ Egg Hatch Inhibition (\%) } & \multicolumn{4}{|c|}{ L1 Mortality (\%) } \\
\hline & & & & & \multirow[b]{2}{*}{6} & \multicolumn{4}{|c|}{$\mathrm{mg} / \mathrm{mL}$} \\
\hline & & 0.75 & 1.5 & 3 & & 0.75 & 1.5 & 3 & 6 \\
\hline $251146^{++}$ & 5.1 & $2 \pm 2$ & $6 \pm 5$ & $73 \pm 15^{*}$ & $98 \pm 2 *$ & $1 \pm 1$ & $3 \pm 1$ & $0 \pm 1$ & $0 \pm 0$ \\
\hline $255305^{++}$ & 21.3 & $6 \pm 2$ & $15 \pm 3$ & $86 \pm 3^{*}$ & $97 \pm 2 *$ & $9 \pm 3$ & $26 \pm 3$ & $46 \pm 13 *$ & $28 \pm 10$ \\
\hline $547080^{++}$ & 11.6 & $2 \pm 1$ & $6 \pm 2$ & $53 \pm 2 *$ & $96 \pm 3 *$ & $6 \pm 1$ & $3 \pm 1$ & $27 \pm 5$ & $80 \pm 19$ \\
\hline B1230 & 7.8 & $0 \pm 3$ & $0 \pm 1$ & $15 \pm 2$ & $96 \pm 19^{*}$ & $1 \pm 1$ & $0 \pm 1$ & $2 \pm 1$ & $17 \pm 19$ \\
\hline $227849^{++}$ & 1.8 & $0 \pm 1$ & $56 \pm 4^{*}$ & $91 \pm 7 *$ & $96 \pm 4^{*}$ & $3 \pm 1$ & $37 \pm 8$ & $60 \pm 24$ & $40 \pm 25$ \\
\hline $232098^{++}$ & 29.6 & $0 \pm 2$ & $3 \pm 3$ & $26 \pm 11$ & $95 \pm 3 *$ & $3 \pm 1$ & $5 \pm 3$ & $8 \pm 16$ & $42 \pm 13$ \\
\hline $233807^{++}$ & 12.4 & $0 \pm 1$ & $0 \pm 1$ & $27 \pm 2 *$ & $94 \pm 3 *$ & $0 \pm 0$ & $2 \pm 1$ & $2 \pm 2$ & $16 \pm 7$ \\
\hline $304067^{++}$ & 12.7 & $0 \pm 2$ & $0 \pm 1$ & $37 \pm 4 *$ & $90 \pm 3^{*}$ & $6 \pm 1$ & $9 \pm 2$ & $11 \pm 4$ & $15 \pm 11$ \\
\hline $380896^{++}$ & -- & $3 \pm 2$ & $6 \pm 1$ & $38 \pm 6^{*}$ & $88 \pm 5^{*}$ & $4 \pm 1$ & $15 \pm 2$ & $38 \pm 6$ & $58 \pm 15^{*}$ \\
\hline $3428^{++}$ & -- & $0 \pm 1$ & $3 \pm 2$ & $36 \pm 4^{*}$ & $86 \pm 9 *$ & $1 \pm 1$ & $3 \pm 1$ & $3 \pm 1$ & $41 \pm 20$ \\
\hline $237278^{++}$ & 5.1 & $20 \pm 4$ & $71 \pm 7 *$ & $94 \pm 3^{*}$ & $83 \pm 7^{*}$ & $0 \pm 1$ & $78 \pm 11$ & $30 \pm 5$ & $40 \pm 25$ \\
\hline
\end{tabular}




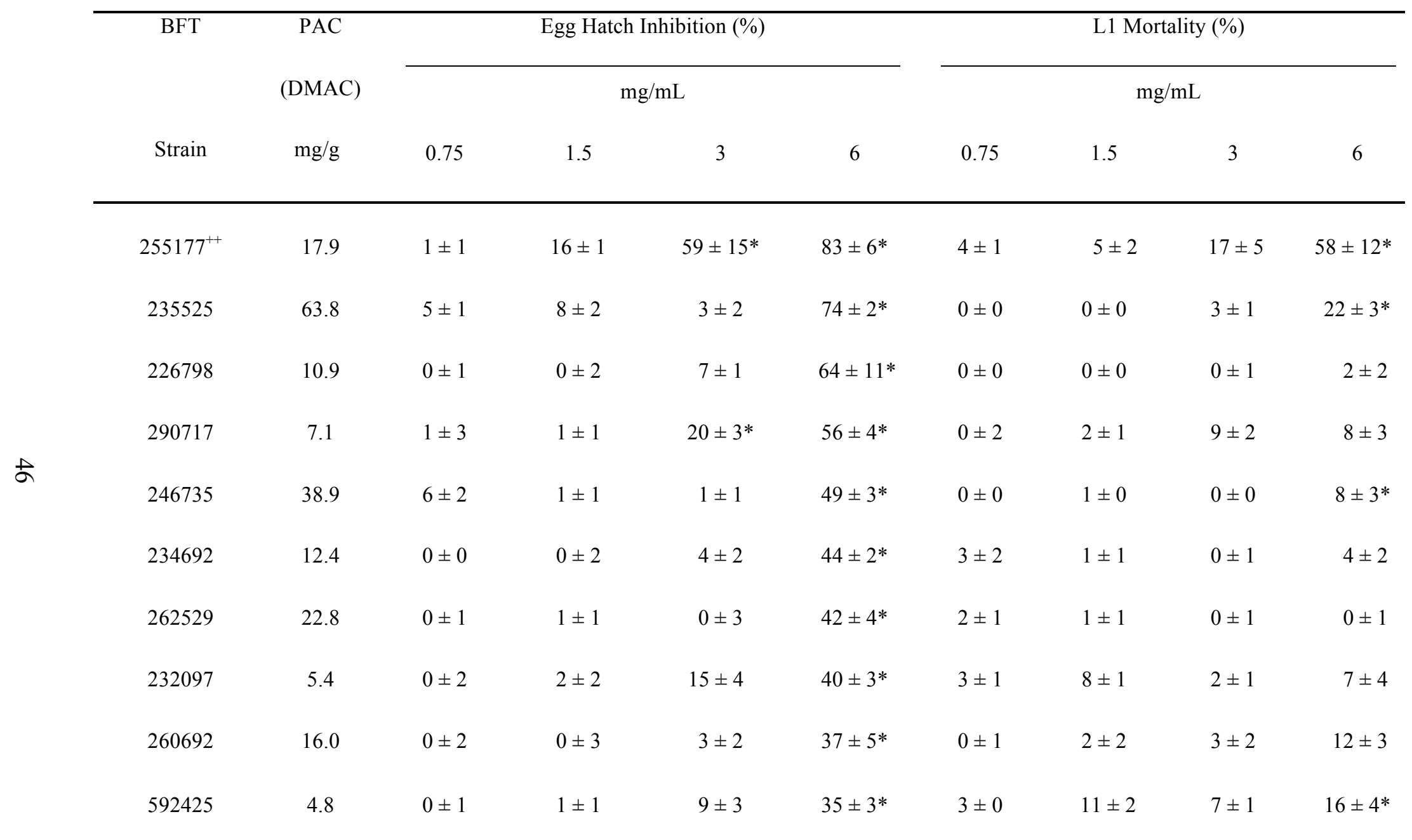

${ }^{1,2}$ Effect of 51 BFT strains against egg hatching and L1 survivability. Eggs ( $\mathrm{n}=100$ eggs/well x 5 wells per treatment) were incubated in BFT-AqE, $(6.0,3.0,1.5$ and $0.75 \mathrm{mg}$ powder $/ \mathrm{mL}$ ), negative controls (water; $0.0 \mathrm{mg} / \mathrm{mL}$ ), and positive controls 
(TBZ; $0.5 \mu \mathrm{g} / \mathrm{mL}$ ) for 24 hours. Eggs were determined hatched or not hatched and eggs that hatched into L1 larvae were determined dead or alive. ${ }^{3}$ Eggs were exposed to Bruce-PAC (6.0, 3.0, 1.5, and $0.75 \mathrm{mg}$ PAC/mL) for 24 hours. Eggs were determined hatched or not hatched and the eggs that hatched into L1 larvae were determined dead or alive. All values are mean \pm SEM, adjusted to zero (negative water control).

${ }^{*} p \leq 0.001$ versus negative control $(0.0 \mathrm{mg} / \mathrm{mL})$.

-- Zero L1 larvae counted; $100 \%$ egg hatch inhibition

${ }^{+}$Insignificant; $<5$ larvae representing percentage.

${ }^{++} p \leq 0.05$, egg hatch inhibition of each strain at $3 \mathrm{mg} / \mathrm{mL}$ vs mean percentage of all negative controls $(0.0 \mathrm{mg} / \mathrm{mL})$. 
Table 3. Lethal concentration that killed $50 \%\left(\mathrm{LC}_{50}\right)$ and $90 \%\left(\mathrm{LC}_{90}\right)(\mathrm{mg} / \mathrm{mL})$ and $95 \%$ confidence interval $(95 \% \mathrm{CI})$ of BFT strains in Haemonchus contortus egg hatch inhibition test.

\begin{tabular}{|c|c|c|}
\hline BFT Strain & $\mathrm{LC}_{50} \mathrm{mg} / \mathrm{mL}(95 \% \mathrm{CI})$ & $\mathrm{LC}_{90} \mathrm{mg} / \mathrm{mL}(95 \% \mathrm{CI})$ \\
\hline Bruce-PAC & $\begin{array}{c}1795.00(119.0- \\
2326124904)\end{array}$ & $\begin{array}{c}22596480.00(33476- \\
1.36 \mathrm{E} 22)\end{array}$ \\
\hline Norcen & $1.04(1.00-1.08)$ & $1.76(1.67-1.88)$ \\
\hline Pardee & $1.55(1.50-1.60)$ & $2.52(2.38-2.68)$ \\
\hline Bruce & $2.50(2.39-2.62)$ & $5.42(5.02-5.92)$ \\
\hline Bull & $1.05(1.01-1.08)$ & $1.48(1.41-1.56)$ \\
\hline Empire & $1.07(1.03-1.10)$ & $1.54(1.47-1.63)$ \\
\hline Leo & $1.07(1.03-1.11)$ & $1.54(1.47-1.63)$ \\
\hline 304523 & $1.18(1.09-1.27)$ & $2.29(2.07-2.59)$ \\
\hline 325379 & $0.66^{*}$ & $0.7 *$ \\
\hline 246730 & $1.72(1.63-1.82)$ & $3.01(2.76-3.34)$ \\
\hline 226796 & $2.19(2.08-2.30)$ & $4.28(3.97-4.67)$ \\
\hline 547080 & $2.53(2.38-2.69)$ & $5.75(5.19-6.49)$ \\
\hline 214110 & $2.65(2.53-2.79)$ & $5.50(5.07-6.04)$ \\
\hline 227315 & $2.05(1.92-2.19)$ & $4.38(3.96-4.95)$ \\
\hline 162456 & $1.47(1.39-1.55)$ & $2.70(2.50-2.97)$ \\
\hline 194228 & $1.78(1.69-1.86)$ & $2.84(2.64-3.09)$ \\
\hline 306182 & $2.11(1.87-2.38)$ & $7.79(6.22-10.61)$ \\
\hline 228286 & $1.11(1.02-1.21)$ & $2.17(1.92-2.56)$ \\
\hline 237278 & $0.92(0.75-1.08)$ & $3.60(2.88-4.99)$ \\
\hline 235525 & $4.36(4.03-4.77)$ & $22.18(18.11-28.36)$ \\
\hline 246735 & $7.85(6.68-9.62)$ & $72.45(47.59-126.64)$ \\
\hline 258446 & $2.12(2.04-2.22)$ & $4.66(4.35-5.04)$ \\
\hline 251143 & $1.37(1.30-1.44)$ & $4.00(3.68-4.41)$ \\
\hline 234670 & $2.37(2.26-2.48)$ & $6.42(5.91-7.05)$ \\
\hline S3149 & $2.54(2.43-2.66)$ & $6.29(5.82-6.87)$ \\
\hline 193725 & $1.59(1.53-1.66)$ & $2.84(2.67-3.06)$ \\
\hline 262530 & $1.86(1.78-1.95)$ & $4.56(4.21-4.99)$ \\
\hline B1230 & $2.92(2.76-3.10)$ & $9.48(8.41-10.90)$ \\
\hline 255305 & $1.55(1.47-1.62)$ & $3.94(3.64-4.31)$ \\
\hline 232098 & $2.55(2.40-2.70)$ & $8.13(7.26-9.28)$ \\
\hline 260692 & $7.55(6.31-9.56)$ & $74.93(46.29-146.24)$ \\
\hline 290717 & $5.16(4.62-5.90)$ & $20.16(15.52-28.65)$ \\
\hline B0730 & $2.89(2.77-3.02)$ & $6.72(6.19-7.38)$ \\
\hline 226798 & $5.09(4.78-5.47)$ & $16.13(13.96-19.16)$ \\
\hline 180171 & $1.76(1.71-1.82)$ & $2.84(2.71-2.99)$ \\
\hline 383689 & $1.87(1.80-1.95)$ & $3.79(3.55-4.07)$ \\
\hline 262529 & $8.04(7.03-9.52)$ & $32.90(24.45-48.87)$ \\
\hline 233807 & $3.11(2.96-3.26)$ & $7.19(6.62-7.92)$ \\
\hline 251146 & $2.09(1.99-2.18)$ & $4.47(4.16-4.86)$ \\
\hline 232097 & $7.30(6.32-8.76)$ & $51.97(35.78-85.53)$ \\
\hline 255177 & $2.52(2.38-2.67)$ & $7.50(6.72-8.52)$ \\
\hline
\end{tabular}




\begin{tabular}{ccc}
\hline BFT Strain & $\mathbf{L C}_{\mathbf{5 0}} \mathbf{~} \mathbf{m g} / \mathbf{m L}(\mathbf{9 5 \%} \mathbf{C I})$ & $\left.\mathbf{L C}_{\mathbf{9 0}} \mathbf{~} \mathbf{m g} / \mathbf{m L} \mathbf{( 9 5 \%} \mathbf{C I}\right)$ \\
\hline 3428 & $2.73(2.58-2.89)$ & $8.96(7.96-10.31)$ \\
234692 & $7.47(6.24-9.44)$ & $81.28(50.03-158.26)$ \\
304067 & $2.83(2.67-2.98)$ & $6.92(6.32-7.68)$ \\
255304 & $1.80(1.72-1.88)$ & $3.99(3.72-4.34)$ \\
310843 & $2.99(2.85-3.15)$ & $6.91(6.34-7.62)$ \\
380896 & $2.70(2.54-2.88)$ & $8.76(7.74-10.14)$ \\
655626 & $1.73(1.66-1.82)$ & $3.67(3.40-4.01)$ \\
592425 & $9.36(7.54-12.54)$ & $97.15(56.56-208.56)$ \\
384882 & $2.15(2.04-2.27)$ & $5.30(4.84-5.89)$ \\
383687 & $2.15(2.05-2.26)$ & $4.93(4.55-5.39)$ \\
227849 & $1.31(1.25-1.37)$ & $2.88(2.67-3.14)$ \\
\hline
\end{tabular}

Calculations based on egg hatch inhibition in $3 \mathrm{mg} / \mathrm{mL}$ BFT-AqE.

*BFT strain 325379 aqeuous extract egg hatch inhibition ranged from $99 \%$ to $100 \%$

(Table 2, the CI could not be calculated by Probit SAS) 
A

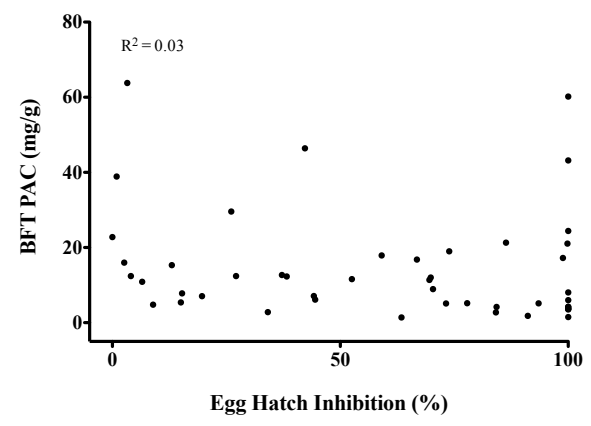

B

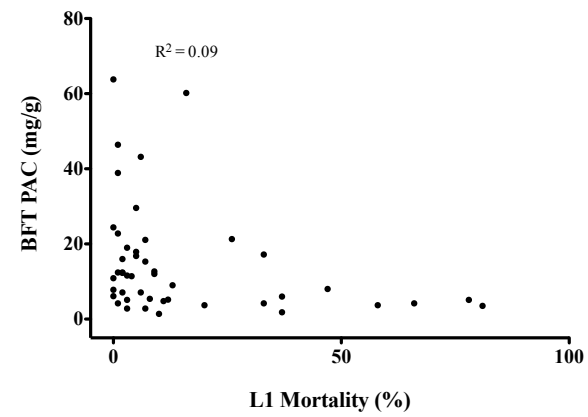

C

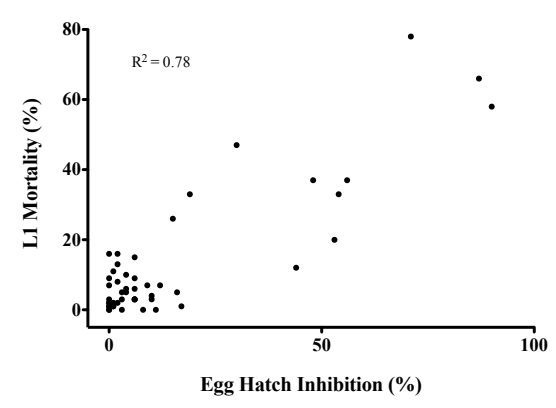

D

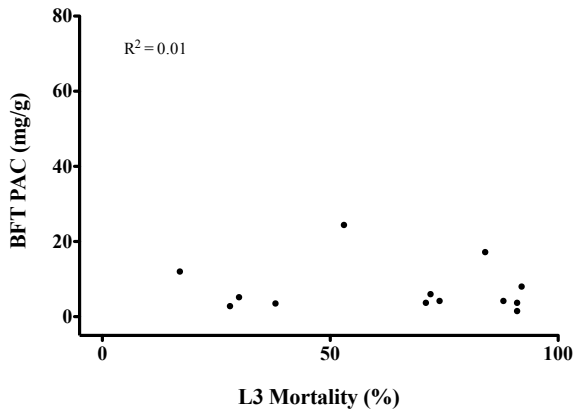

$\mathbf{E}$

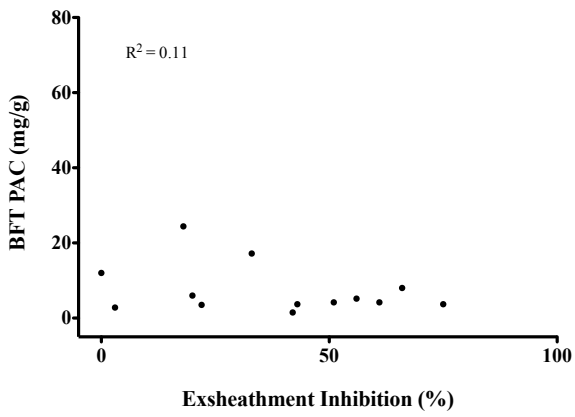

$\mathbf{F}$

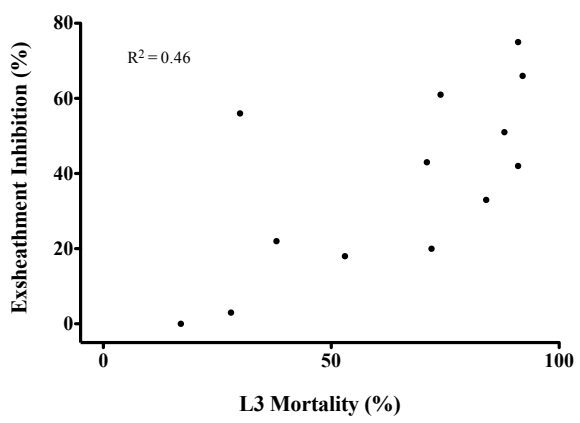

Figure 2. Aqueous extracts ( $1.5 \mathrm{mg}$ powder $/ \mathrm{mL})$ affect on egg hatching, $\mathrm{L} 1$ and L3 viability, and the exsheathment process compared to levels of PAC present in the BFT powders. There was no correlation between the BFT powder PAC concentrations and the efficacy of BFT-AqE against egg hatching $\left(R^{2}=0.03, \mathbf{A}\right)$, $\mathrm{L} 1\left(R^{2}=0.09, \mathbf{B}\right)$ and L3 $\left(R^{2}=0.01, \mathbf{D}\right)$ larvae, and exsheathment $\left(R^{2}=0.11, \mathbf{E}\right)$. There was a significant correlation between L1 mortality and egg hatch inhibition $\left(R^{2}=0.78, \mathbf{C}\right)$ as well as a significant correlation between L3 mortality and exsheathment inhibition $\left(R^{2}=0.46, \mathbf{F}\right)$. 
Table 4. Aqueous extracts of the top 13 BFT strains and purified Bruce-PAC extract tested against Haemonchus contortus L3 mortality and exsheathment inhibition. ${ }^{1}$

\begin{tabular}{|c|c|c|c|}
\hline \multirow[t]{2}{*}{$\begin{array}{c}\text { BFT } \\
\text { Variety }\end{array}$} & PAC & L3 Mortality (\%) & $\begin{array}{l}\text { Exsheathment } \\
\text { Inhibition (\%) }\end{array}$ \\
\hline & $\mathrm{mg} / \mathrm{g}$ & $25 \mathrm{mg} / \mathrm{mL}$ & $25 \mathrm{mg} / \mathrm{mL}$ \\
\hline Pardee $^{+}$ & 8.0 & $92 \pm 2^{*}$ & $66 \pm 12 *$ \\
\hline 228286 & 3.7 & $91 \pm 2^{*}$ & $75 \pm 10^{*}$ \\
\hline 655626 & 4.2 & $74 \pm 5^{*}$ & $61 \pm 9^{*}$ \\
\hline Empire $^{+}$ & 4.2 & $88 \pm 4^{*}$ & $51 \pm 14^{*}$ \\
\hline 325379 & 1.5 & $91 \pm 3^{*}$ & $42 \pm 14$ \\
\hline $\mathrm{Bull}^{+}$ & 3.7 & $71 \pm 4^{*}$ & $43 \pm 9^{*}$ \\
\hline 304523 & 17.2 & $84 \pm 4^{*}$ & $33 \pm 11$ \\
\hline Norcen $^{+}$ & 3.5 & $38 \pm 6^{*}$ & $22 \pm 9$ \\
\hline $\mathrm{Leo}^{+}$ & 6.0 & $72 \pm 6^{*}$ & $20 \pm 7$ \\
\hline 193725 & 24.4 & $53 \pm 5^{*}$ & $18 \pm 6$ \\
\hline 251143 & 5.2 & $30 \pm 5^{*}$ & $56 \pm 5^{*}$ \\
\hline 306182 & 2.8 & $28 \pm 6^{*}$ & $3 \pm 3$ \\
\hline \multirow[t]{2}{*}{ Bruce $^{+}$} & 12.0 & $17 \pm 5$ & $0 \pm 2$ \\
\hline & & $6 \mathrm{mg} / \mathrm{mL}$ & $6 \mathrm{mg} / \mathrm{mL}$ \\
\hline
\end{tabular}

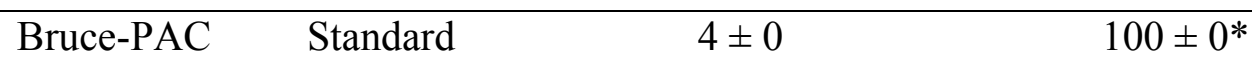

${ }^{1}$ Haemonchus contortus (L3) were incubated for $24 \mathrm{hrs}$ in $25 \mathrm{mg}$ powder/mL BFT$\mathrm{AqE}$ of the top ranked BFT varieties. Larvae were also exposed to Bruce-PAC $(6.0 \mathrm{mg} / \mathrm{mL})$ for $24 \mathrm{hrs}$. Larvae were determined dead or alive and then exposed to $\mathrm{CO}_{2}$ bubbling and incubated for another $18 \mathrm{hrs}$. All larvae were determined dead or alive, and live larvae were determined sheathed or exsheathed. Negative controls were water $(0.0 \mathrm{mg} / \mathrm{mL})$, positive control was Thiabendazole (TBZ, 0.5 $\mu \mathrm{g} / \mathrm{mL}$ ), values are mean $\pm \mathrm{SEM}$, and have been adjusted to negative control (water).

${ }^{+}$Commercially available in the U.S. in 2017.

${ }^{*} p \leq 0.001$ versus negative control $(0.0 \mathrm{mg} / \mathrm{mL})$. 


\subsection{L3 mortality and exsheathment inhibition assay}

The top performing strains were rank ordered based on the efficacy of each variety against egg hatch inhibition and L1 mortality. Five of the top 12 strains were commercially available in the United States. 'Bruce' was added as the $13^{\text {th }}$ strain assayed so that all current commercial varieties would be tested. Across the thirteen aqueous extracts tested, efficacy ranged from 0 to $75 \%$ exsheathment inhibition, and 17 to $92 \%$ L3 mortality at a concentration of $25 \mathrm{mg} / \mathrm{mL}$ BFT-AqE

(Table 4). When comparing the L3 mortality percentages and exsheathment inhibition percentages of the 13 varieties, at $1.5 \mathrm{mg}$ powder $/ \mathrm{mL}$, there was a significant correlation between L3 mortality and exsheathment inhibition $\left(R^{2}=\right.$ 0.46) (Fig. 2. F). The Bruce-PAC that was screened, exhibited 4\% L3 mortality and $100 \%$ exsheathment inhibition at a concentration of $6 \mathrm{mg} \mathrm{PAC} / \mathrm{mL}$ (Table 4).

The top 12 strains, all inhibited egg hatch by $99 \%$ or $100 \%$ at $6 \mathrm{mg}$ powder $/ \mathrm{mL}$. At a concentration of $3 \mathrm{mg}$ powder $/ \mathrm{mL}, 10$ of the 13 strains exhibited $>$ $84 \%$ egg hatch inhibition. The three strains that were $<80 \%$ effective against egg hatching included 251143 (78\%), 'Bruce' (70\%), and 306182 (34\%). Compared to L3 mortality and exsheathment inhibition assay results, 'Bruce' $\left(13^{\text {th }}\right), 306182\left(12^{\text {th }}\right)$, and $251143\left(11^{\text {th }}\right)$ were the strains ranked lowest. 'Bruce' did have the lowest percent exsheathment inhibition ( $0 \%$ ), followed closely by 306182 , with $3 \%$ exsheathment inhibition. 'Bruce' also exhibited 17\% L3 mortality, while strains 306182 and 251143 exhibited 28\% and 30\% L3 mortality respectively.

The crude Bruce-AqE extract, at $25 \mathrm{mg}$ powder/mL exhibited $0 \%$ exsheathment inhibition, whereas the purified Bruce-PAC extract, at only $6 \mathrm{mg} / \mathrm{mL}$ 
exhibited $100 \%$ exsheathment inhibition, and $70 \%$ exsheathment inhibition at the lowest concentration of $0.75 \mathrm{mg} / \mathrm{mL}$ Bruce-PAC (data not shown). As for L3 mortality, neither extract exhibited high efficacy. The Bruce-AqE (25 mg powder $/ \mathrm{mL}$ ) exhibited 17\% L3 mortality, while the Bruce-PAC (6 mg PAC $/ \mathrm{mL}$ ) exhibited 4\% L3 mortality.

\subsection{Correlation between anthelmintic efficacy and PAC content}

The anthelmintic efficacy of the 51 BFT-AqE extracts against egg hatch and L1 mortality compared to the PAC content of each BFT powder, showed no significant correlation (egg hatch percentages at $1.5 \mathrm{mg}$ powder $/ \mathrm{mL}$ BFT-AqE, $R^{2}=$

0.03 (Fig. 2. A); L1 mortality percentages at $1.5 \mathrm{mg}$ powder $/ \mathrm{mL}$ BFT-AqE, $R^{2}=0.09$ (Fig. 2. B)). The anthelmintic efficacy of the top 13 BFT-AqE extracts against L3 viability and exsheathment also did not show any correlation to PAC content of the BFT powders (L3 mortality percentages at $25 \mathrm{mg}$ powder $/ \mathrm{mL}$ BFT-AqE, $R^{2}=0.01$ (Fig. 2. D); L3 exsheathment inhibition percentages at $25 \mathrm{mg}$ powder/mL BFT-AqE, $R^{2}=0.11($ Fig. 2. E))

\section{Discussion}

This study determined the potential anti-parasitic effect of 51 strains of birdsfoot trefoil by in vitro screening against $H$. contortus egg hatch, L1 and L3 viability, and exsheathment. It was found that PAC content of BFT powders did not directly correlate with the anthelmintic efficacy of aqueous BFT powder extracts. Structural characteristics of PAC need to be further investigated for their potential influence on varietal bioactivity. 


\subsection{Diversity of plant secondary metabolites and extracts}

All fifty-one BFT strains were grown and harvested at the University of Rhode Island, during the summer. All samples were collected during the same season in order to lower any environmentally associated variables. The concentration of PAC is equally influenced by plant genetics as well as by the environment (Grabber et al., 2014). In a study comparing these two factors, it was found that the maturity of the BFT cultivar at harvest was positively associated with PAC content (Grabber et al., 2014). In general, there is vast diversity in biochemical, morphological, and agronomic characteristics of BFT strains (Steiner et al., 2001) and PAC concentrations across BFT strains have been shown to differ substantially (Grabber et al., 2014). Similar to these previous investigations, PAC content across the 51 strains of BFT used in this study ranged widely, from 1.4 to $63.8 \mathrm{mg} \mathrm{PAC/g}$ powder (Reed et al., in

preparation). Condensed tannin concentrations were not consistent for each variety when comparing results from Reed et al. (in preparation) and previous studies (Roberts et al., 1993; Grabber et al., 2014; GRIN database). This discrepancy may be due to the method of PAC content determination or age at harvest.

Previous studies have commonly used the butanol- $\mathrm{HCl}$ method to determine PAC content (Grabber et al., 2014), however the analysis of the BFT strains used in this study was performed using DMAC analysis (Reed et al., in preparation). It has been reported that the butanol- $\mathrm{HCl}$ assay includes sources of interference, such as anthocyanins, while the DMAC method is more accurate and sensitive for PAC (Feliciano et al., 2012a). Since the DMAC method is only able to quantify 
soluble/extractable PAC, a subsequent analysis was run on the residue, using the $\mathrm{BuOH}-\mathrm{HCl}$ method to quantify the insoluble PAC (Reed et al., in preparation).

Unlike most previous work with crude plant extracts, this investigation of BFT did not use organic solvents to develop the extracts used in screening the BFT strains. The extraction method used for this study was similar to other studies using aqueous extractions: soaking plant-derived products in water (Adamu et al., 2010; Ferreira et al., 2013) and testing the extracts against $H$. contortus. Previous investigators of crude extracts showing anthelmintic efficacy against $H$. contortus attributed this activity to alkaloids, saponins (Adamu et al., 2010), and phenolic compounds (Ferreira et al., 2013). A key difference in the preparation of other aqueous extracts is the use of boiling water (Ferreira et al., 2013), unlike the preparation of the BFT-AqE used in this study, utilizing room temperature water. Hot water was not used, simply to prevent complexing of secondary compounds, specifically PAC, under high heat (Reed, 1995). However, the use of boiling water would have more thouroughly disrupted the cell membranes, potentially extracting compounds that room temperature water would not.

In addition to the crude aqueous extracts, one purified PAC extract was prepared for this study to compare the efficacy of the BFT-AqE to the efficacy of Bruce-PAC. Only one variety ('Bruce') was chosen based upon the amount of freezedried plant matter required to make enough PAC for a bioassay. It was also selected because it was used as the initial standard during phytochemical analyses based upon preliminary analysis of its high PAC content compared to the other five commercial varieties. This extraction of PAC differs from both the crude aqueous and the crude 
organic extraction methods because it isolates only PAC, excluding other impurities. This method includes an organic extraction process, but continues to purify the extract by elution through a Sephadex LH-20 column to separate the PAC from the other compounds. This purification of PAC has been used in some studies (Molan and Faraj, 2010), but many previous investigations have used crude organic extracts (Hernández-Villegas et al., 2011; Monteiro et al., 2011).

\subsection{Bruce-PAC versus BFT-AqE}

The Bruce-PAC extract was pure isolated PAC and it was expected to exhibit higher egg hatch inhibition rates, but the opposite was observed. Both extracts performed similarly against L1 larvae, with greater than $84 \%$ mortality. Another difference between these extracts was observed in the exsheathment inhibition assay. The efficacy differences noted between the crude aqueous extract and the purified proanthocyanidin extract suggest that there are other secondary compounds contributing to the bioactivity observed in the BFT-AqE extracts that could be inhibiting egg hatching, but have much less of an effect on exsheathment. Both extracts appeared to affect the mortality of L1 larvae similarly, and both had slight deleterious effects on the L3 larvae. The notable finding that the PAC extracted from the BFT variety 'Bruce' did not inhibit egg hatching contradicts what was expected and what was seen using the BFT-AqE. In a study using purified proanthocyanidin extracts (utilizing the Sephadex LH-20 column to isolate PAC), one of which was from Lotus corniculatus, against egg hatching of Teladorsagia circumcincta, $32 \%$ egg hatch inhibition in $900 \mu \mathrm{g} / \mathrm{mL}$ was observed (Molan and Faraj, 2010). These results differ from the Bruce-PAC extract used in this investigation, since this study used 
notably higher concentrations $(6 \mathrm{mg} \mathrm{PAC} / \mathrm{mL})$ and didn't reach egg hatch inhibition values near the $32 \%$ observed by Molan and Faraj (2010) at a concentration of 0.9 $\mathrm{mg} / \mathrm{mL}$. Although these differences should be acknowledged, it should also be taken into consideration that the variety of BFT used in the study with T. circumcincta was not reported, and based off of the PAC content differences observed in BFT strains used in this study (Reed et al., in preparation), BFT strain does effect efficacy. However, because the extract should solely contain isolated PAC, the difference would not lie in the amount of PAC present in that variety, but rather suggest that the structure of the PAC is different between the two varieties used at the same concentration. Additionally, the species of GIN tested should be considered, as BFT PAC could potentially have more of an effect against $T$. circumcincta than against $H$. contortus. Furthermore, the extraction process may have had an effect on the bioactivity of the BFT PAC, as part of the process involves evaporation under vacuum at $35^{\circ} \mathrm{C}$ (Krueger et al., 2016; Feliciano et al., 2012b), but has not shown to alter the structure of the material and is used as a standard method for preparing PAC standard reference material.

\subsection{Biological relevance of egg hatch and L1 mortality assay}

The BFT aqueous extracts and the single Bruce-PAC extract from the 'Bruce' BFT variety were screened using the egg hatch and L1 mortality assay in order to assess any anthelmintic differences between the BFT strains. For this assay, eggs and larvae were incubated in BFT-AqE extracts for 24 hours, compared to other egg hatch assays that incubated eggs in PAC for up to 48 hours (Vargas-Magaña et al., 2014). In the current study, results were recorded at 24 hours instead of 48 , because eggs were 
$>90 \%$ hatched in controls at 24 hours. The variability in anthelmintic activity observed across the fifty-one BFT strains was also observed in studies of other plant extracts. An in vitro screening of forty plant extracts against Haemonchus contortus investigated the effects of various plants against egg hatching and larval migration (Acharya et al., 2014). Plant extracts were prepared by methanol extraction (Acharya et al., 2014), different from the aqueous extract used in this investigation of BFT. Nonetheless, the effect of the forty plant extracts on Haemonchus contortus ranged from 3.1\% to $100 \%$ egg hatch inhibition (Acharya et al., 2014). This range across different species of plants was similar to the range observed in this study of 51 strains of Lotus corniculatus. The anthelmintic diversity within this one plant species proved to be as diverse as the anthelmintic diversity across forty different species. Although there was anthelmintic diversity, one consistency observed was a positive correlation between egg hatch inhibition and L1 mortality. Therefore, the egg hatch assay can be utilized as a screening tool to distinguish highly efficacious varieties from ineffectual strains of BFT in vitro.

The egg hatch and L1 mortality assays may be indicative of in vivo biological activity against the hatching of eggs and L1 survivability in feces on pasture. Targeting this stage of the parasite could potentially reduce contamination on pasture and ultimately decrease infectivity rates of grazing small ruminants. Previous studies have shown that PAC is not absorbed from the gastrointestinal tract; consequently, it is expelled in the feces of animals consuming PAC rich diets (Terrill et al., 1994), ultimately coming in contact with eggs and hatched L1 larvae in the feces. In vivo, the hatch rate of GIN eggs was lower in sheep fed PAC rich plants compared to sheep fed 
PAC free diets (Niezen et al., 2002). Also in vivo, GIN egg hatch rates were reduced when small ruminants were drenched with wattle tannin, a commercially sold PAC extract powder from the bark of Acacia mearnsii (Max, 2010). If the concentrations used in this investigation of BFT were to be used in vivo at the highest concentration tested $(6 \mathrm{mg} / \mathrm{mL})$, it would be suggested that an animal would need to consume 48 grams (dry matter weight; DM) of BFT (6 mg/mL x 8,000 mL (assuming 8 L rumen capacity)) to be equivalent to the concentration that the eggs and L1 larvae were exposed to in vitro. For example, feeding BFT hay (90\% DM) would require consumption of $53 \mathrm{~g}$ BFT hay, to ingest $48 \mathrm{~g}$ DM. This amount of BFT is a feasible quantity for a producer to administer and for an animal to consume.

Despite the evidence that PAC has deleterious effects on GIN, the mechanism of action is unknown. A study investigating the role of tannins on the inhibition of egg hatching, found acetone-water plant extracts (Lysiloma latisiliquum, Laguncularia racemosa, Rizophora mangle, Avicennia germinans, Theobroma cacao) affected hatching of $H$. contortus eggs mainly by blocking eclosion of larvae from eggs (Vargas-Magaña et al., 2014). Tannins may be a contributing factor in the efficacy of the BFT-AqE extracts against egg hatch and L1 survivability in this study of BFT, but there was no correlation between DMAC PAC concentration (mg PAC/g powder) of freeze dried BFT powders and the inhibition of egg hatch using aqueous extracts of BFT powder. The aqueous extract may not have extracted all of the available PAC in the plant matter, as the extent of cell membrane disruption was the grinding process. Water-soluble PAC or other secondary compounds might have also extracted in the aqueous extract, contributing to the anthelmintic efficacy observed. Additionally, 
aside from PAC content and other secondary compounds, the structure of the PAC could also potentially influence the level of bioactivity that a specific variety or cultivar exhibits. Based on the results for Bruce-PAC and Bruce-AqE extracts, the most plausible explanation for the observed differences is the presence of secondary compounds working synergistically with PAC.

\subsection{Biological relevance of $L 3$ mortality and exsheathment inhibition assay}

Although the egg hatch assay has relevance on pasture, the exsheathment assay is of further utility and significance. The exsheathment process is a crucial step in the development of $H$. contortus, as the larvae must undergo a molting of their sheath to eventually develop into an adult worm. If the process of exsheathment is obstructed, L3 are eliminated with the feces and die (von Son-de Fernex et al., 2012). The larval exsheathment inhibition assay is the most relevant assay to reproduce the process of exsheathment that occurs in vivo (Klongsiriwet et al., 2015).

Prior to initiating exsheathment, all larvae were exposed to BFT-AqE extract for 24 hours. This incubation period was determined based on the flow rate time of digesta in sheep. The mean half-time of sheep fed $800 \mathrm{~g}$ lucerne chaff per day using chromium-51 with EDTA marker was $\approx 13.5$ hours in the reticulo-rumen, while the mean half-time of sheep fed the same diet but using cerium-144 - praseodymium-144 marker was $\approx 10$ hours in the reticulo-rumen (Grovum and Williams, 1977). With a calculated flow rate time up to 20.1 - 27 hours, this study of BFT exposed the L3 to the BFT-AqE extracts for 24 hours prior to initiating artificial exsheathment.

Our results indicated a positive correlation between L3 mortality and inhibition of exsheathment. This means that if a BFT strain exhibited high deleterious effects 
against the L3 viability, it also exhibited inhibitory effects against exsheathment; similarly if a BFT strain didn't have any effect on L3 larvae it also didn't effect exsheathment. Investigators testing the influence of PAC containing plant extracts on the exsheathment process of L3 H. contortus, observed inhibition of exsheathment using all four plant species tested, but did not report the viability of the L3 $H$. contortus post exsheathment (Alonso-Díaz et al., 2008). Investigators using PAC-rich sainfoin extract observed a dose dependent response, in which exsheathment was delayed significantly at $0.3 \mathrm{mg} / \mathrm{mL}$ and highly inhibited at $1.2 \mathrm{mg} / \mathrm{mL}$, but also did not report post exsheathment viability percentages (Brunet et al., 2007). Similar to Brunet et al. (2007), in our study of BFT, different levels of efficacy were observed depending on the variety of BFT-AqE tested, and there was also a dose dependent response in the Bruce-PAC treatment (data not shown). One major difference between these previously published studies and this investigation of BFT is the method of artificial exsheathment, which may have had detrimental effects on the viability of the larvae used in previous assays. Initiation of exsheathment was performed using a $\mathrm{CO}_{2}$ technique (Conder and Johnson, 1996), different from most other exsheathment assays that use contact with a solution of sodium hypochlorite $(2 \% \mathrm{w} / \mathrm{v})$ and sodium chloride (16.5\% w/v) in PBS (Alonso-Díaz et al., 2008; von Son-de Fernex et al., 2012). Despite the popularity of using sodium hypochlorite, artificial exsheathment for this study of BFT was initiated by exposure to $\mathrm{CO}_{2}$ in EBSS, bubbled through Parafilm $\mathrm{M} \circledast$ (Conder and Johnson, 1996). This method has been shown to produce similarly high exsheathment rates when compared to sodium hypochlorite. In one study comparing these two methods, the infectivity rates of sodium hypochlorite treated 
worms was reduced significantly compared to that of larvae exsheathed using EBSS and carbon dioxide (Conder and Johnson, 1996). For this investigation of BFT, the L3 larvae were exposed to the $\mathrm{CO}_{2}$ in EBSS, because $H$. contortus exposed to EBSS and $\mathrm{CO}_{2}$ produced higher levels of exsheathment than those exsheathed with distilled water (Conder and Johnson, 1996). Therefore, in this study, EBSS and $\mathrm{CO}_{2}$ were used to ensure viability of larvae as well as high exsheathment rates.

Efficacy was compared of the top 13 varieties in both the egg hatch and L1 mortality assay and the L3 mortality and exsheathment assay. Although the bottom ranked strains remained poor performers in both assays, the 10 strains that were $>$ $84 \%$ effective against egg hatching, exhibited additional variation when screened for L3 mortality and exsheathment inhibition. The top 10 ranked strains ranged from 18 to $75 \%$ exsheathment inhibition and 38 to $92 \% \mathrm{~L} 3$ mortality at $25 \mathrm{mg}$ powder $/ \mathrm{mL}$ BFT-AqE. This variation could be attributed to the different developmental stages of the parasite and that the mechanism of action and effectiveness may vary when comparing egg hatching, L1, L3, and the exsheathment process. It should also be noted that results from the egg hatch and L1 mortality assays were reflective of exposure to $6 \mathrm{mg}$ powder/mL BFT-AqE and lower, while the results from the L3 mortality and exsheathment inhibition assays were of L3 larvae exposure to $25 \mathrm{mg}$ powder/mL, a much higher concentration. Although the concentrations were different, the ranking may have differed due to the developmental stage of the parasite exposed to the extracts. The activity observed against egg hatching and L1 larvae was not reflective of how those same extracts performed against L3 larvae and exsheathment and studies have suggested that antiparasitic activity can differ depending on the 
developmental stage of the parasite (Hoste et al., 2006). The mechanism of action behind how secondary compounds negatively affect $H$. contortus at different life stages remains unknown and might provide explanation for the differences in efficacy observed in this study, against egg hatching, larval stages, and exsheathment.

If the concentration at which the L3 mortality and exsheathment inhibition assays were performed at was to be used in vivo, $25 \mathrm{mg} / \mathrm{mL}$ would be equivalent to the animal consuming $200 \mathrm{~g}(\mathrm{DM})$ of BFT $(25 \mathrm{mg} / \mathrm{mL} \times 8,000 \mathrm{~mL}$ (assuming $8 \mathrm{~L}$ of rumen capacity)). If feeding as hay (90\% DM), an animal would need to consume 222 $\mathrm{g}$, a feasible amount to administer and for the animal to consume.

\subsection{Significance of PAC content and structural characteristics}

The majority of studies investigating the anthelmintic efficacy of plant extracts use crude organic extracts (Acharya et al., 2014; von Son-de Fernex et al., 2012; Klongsiriwet et al., 2015). Investigations using crude organic extracts have credited other secondary compounds present in the extracts, beside PAC, that may also be contributing to the anthelmintic efficacy observed (Acharya et al., 2014; HernándezVillegas et al., 2011; Monteiro et al., 2011). Supporting this hypothesis, investigators studying the effects of flavonoid monomers and condensed tannins on exsheathment inhibition concluded that synergistic anthelmintic effects exist between flavonoid monomers and condensed tannins. They proposed that anthelmintic efficacy of condensed tannins could be significantly enhanced by adding quercetin or luteolin to the bioassay (Klongsiriwet et al., 2015). Secondary metabolites that have been documented as anti-parasitic include saponins, non-protein amino acids, alkaloids, polyphenols, glycosides, and lignins (Githiori et al., 2006). Since the BFT-AqE is a 
crude extraction and contains water-soluble secondary metabolites, including PAC, this could be contributing to the efficacy of the individual strains and might explain why no correlation was observed between BFT aqueous extract efficacy and BFT powder PAC content. Additionally, there is the potential that PAC structure is playing a role in anthelmintic efficacy and that structural characteristics of PAC differ between varieties.

The top 13 varieties were analyzed using MALDI-TOF MS to provide structural characteristics for top performing and low performing varieties. These analyses indicated B-type proanthocyanidin linkages, not A-type (Reed et al., in preparation). This is not surprising as the B-type bonds are the more common of the two (Howell, 2007) and results are consistent with previous MALDI-TOF mass spectra analyses of Lotus corniculatus (cv. Fergus), in which proanthocyanidins presented only as B-type procyanidins and prodelphinidins (Hedqvist et al., 2000; Monagas et al., 2010). The structure of PAC includes more than just linkage differences, such as distinctive patterns of B-ring hydroxylation (Hedqvist et al., 2000). Structural analyses collected on proanthocyanidins from sorghum, grapes, and C. calothyrsus (Hedqvist et al., 2000; Krueger et al., 2000) indicate that an array of structures can occur within one species. Seven BFT cultivars (Viking, Dawn, Fergus, GA1, Norcen, Empire, and Leo) were compared, and differences in PAC content as well as PAC composition between the cultivars were reported (Hedqvist et al., 2000).

The phytochemical investigation of the 51 varieties of BFT used in this study, reported both PAC content differences as well as PAC structural differences (Reed et al., in preparation). Analysis of the ground BFT powder indicated B-type linkages 
(not A-type linkages) and the presence of glycosylated PAC (Reed et al., in preparation). Differences in PAC content or structural characteristics including linkages, glycosylation, and hydroxylation between varieties may have the potential to correlate to anthelmintic efficacy and contribute to our understanding of the mechanism of action of proanthocyanidins against GIN parasites.

The diversity seen in the PAC content of the powders and the diversity observed in the in vitro screening tests (using aqueous extractions) suggested that the PAC levels of the individual BFT strains did not directly correlate to the anthelmintic efficacy of the aqueous extract against $H$. contortus egg hatching, L1 and L3 mortality, and exsheathment. Although there was no correlation with PAC content, structural characteristics of PAC may be influencing bioactivity. Such information may provide insight into the investigation of plants extracts and forages used as alternative methods to control $H$. contortus in small ruminants. Being able to correlate a structural characteristic found within PAC to high anthelmintic efficacy could provide a technique that would determine the potential anthelmintic efficacy of a plant species or variety based on PAC structure and make it possible to breed strains specifically for anthelmintic use.

\section{Conclusions}

This study provided a broad anthelmintic and phytochemical overview and comparison of the variation across 51 strains of Lotus corniculatus. The anthelmintic diversity observed across the 51 strains of BFT, combined with finding that this antiparasitic activity did not correlate to the PAC content of the original plant material, suggests a potential role of other secondary compounds or varietal PAC structural 
differences. Using isolated PAC extracts from other BFT strains could differentiate between the potential role of other secondary compounds and structural differences. Based on in vitro anthelmintic efficacies of the 51 BFT strains, the commercially available strains could potentially be recommended to a producer as a medicinal forage to plant and allow their animals to graze on. Further investigation is warranted through additional in vitro testing of purified proanthocyanidin extracts to compare efficacies to crude aqueous extracts and PAC concentrations and structure.

\section{Acknowledgements}

The authors of this study would like to thank Dr. Rodrigo Feliciano for his training and expertise in DMAC and MALDI-TOF MS analyses. The authors would also like to thank the many individuals who contributed to the harvesting, collection, and freeze-drying of the BFT plant material used for this study. 


\section{References}

Acharya, J., Hildreth, M.B., Reese, R.N., 2014. In vitro screening of forty medicinal plant extracts from the United States Northern Great Plains for anthelmintic activity against Haemonchus contortus. Vet. Parasitol. 201 (1), $75-81$.

Adamu, M., Oshadu, O.D., Ogbaje, C.I., 2010. Anthelminthic efficacy of aqueous extract of Acanthus montanus leaf against strongylid nematodes of small ruminants. Afr. J. Tradit. Complement. Altern. Med. 7 (4), 279-285.

Alonso-Diaz, M.A., Torres-Acosta, J.F., Sandoval-Castro, C.A., AguilarCaballero, A.J., Hoste, H., 2008. In vitro larval migration and kinetics of exsheathment of Haemonchus contortus larvae exposed to four tropical tanniniferous plant extracts. Vet. Parasitol. 153 (3-4), 313-319, 10.1016/j.vetpar.2008.01.042 [doi].

Assis, L.M., Bevilaqua, C.M.L., Morais, S.M., Vieira, L.S., Costa, C.T.C., Souza, J.A.L., 2003. Ovicidal and larvicidal activity in vitro of Spigelia anthelmia Linn. extracts on Haemonchus contortus. Vet. Parasitol. 117 (1-2), 43-49, http://dx.doi.org/10.1016/j.vetpar.2003.07.021.

Brunet, S., Aufrere, J., El Babili, F., Fouraste, I., Hoste, H., 2007. The kinetics of exsheathment of infective nematode larvae is disturbed in the presence of a tannin-rich plant extract (sainfoin) both in vitro and in vivo. Parasitology 134 (Pt 9), 1253-1262, S0031182007002533 [pii].

Conder, G.A., Johnson, S.S., 1996. Viability of infective larvae of Haemonchus contortus, Ostertagia ostertagi, and Trichostrongylus colubriformis 
following exsheathment by various techniques. J. Parasitol. 82 (1), 100102.

Ferguson, S. 2016. Agronomic characteristics of birdsfoot trefoil (Lotus corniculatus L.) in the northeast United States. Thesis. Manuscript in preparation.

Feliciano, R.P., Shea, M.P., Shanmuganayagam, D., Krueger, C.G., Howell, A.B., Reed, J.D., 2012a. Comparison of isolated cranberry (Vaccinium macrocarpon Ait.) proanthocyanidins to catechin and procyanidins A2 and B2 for use as standards in the 4-(dimethylamino) cinnamaldehyde assay. J. Agric. Food Chem. 60 (18), 4578-4585.

Feliciano, R.P., Krueger, C.G., Shanmuganayagam, D., Vestling, M.M., Reed, J.D., 2012b. Deconvolution of matrix-assisted laser desorption/ionization time-of-flight mass spectrometry isotope patterns to determine ratios of Atype to B-type interflavan bonds in cranberry proanthocyanidins. Food Chem. 135 (3), 1485-1493, 10.1016/j.foodchem.2012.05.102 [doi].

Ferreira, L.E., Castro, P.M., Chagas, A.C., Franca, S.C., Beleboni, R.O., 2013. In vitro anthelmintic activity of aqueous leaf extract of Annona muricata $\mathrm{L}$. (Annonaceae) against Haemonchus contortus from sheep. Exp. Parasitol. 134 (3), 327-332, 10.1016/j.exppara.2013.03.032 [doi].

Githiori, J.B., Athanasiadou, S., Thamsborg, S.M., 2006. Use of plants in novel approaches for control of gastrointestinal helminths in livestock with emphasis on small ruminants. Vet. Parasitol. 139 (4), 308-320, http://dx.doi.org/10.1016/j.vetpar.2006.04.021. 
Grabber, J., Riday, H., Cassida, K., Griggs, T., Min, D., MacAdam, J., 2014. Yield, morphological characteristics, and chemical composition of European-and Mediterranean-derived birdsfoot trefoil cultivars grown in the colder continental United States. Crop Sci. 54 (4), 1893-1901.

Grovum, W.L., Williams, V.J., 1977. Rate of passage of digesta in sheep. 6. The effect of level of food intake on mathematical predictions of the kinetics of digesta in the reticulorumen and intestines. Br. J. Nutr. 38 (3), 425-436, S0007114577000555 [pii].

Heckendorn, F., Haring, D.A., Maurer, V., Senn, M., Hertzberg, H., 2007. Individual administration of three tanniferous forage plants to lambs artificially infected with Haemonchus contortus and Cooperia curticei. Vet. Parasitol. 146 (1-2), 123-134, S0304-4017(07)00052-0 [pii].

Hedqvist, H., Mueller-Harvey, I., Reed, J.D., Krueger, C.G., Murphy, M., 2000. Characterisation of tannins and in vitro protein digestibility of several Lotus corniculatus varieties. Anim. Feed Sci. Technol. 87 (1), 41-56. Hernández-Villegas, M.M., Borges-Argáez, R., Rodriguez-Vivas, R.I., TorresAcosta, J.F.J., Méndez-Gonzalez, M., Cáceres-Farfan, M., 2011. Ovicidal and larvicidal activity of the crude extracts from Phytolacca icosandra against Haemonchus contortus. Vet. Parasitol. 179 (1-3), 100-106, http://dx.doi.org/10.1016/j.vetpar.2011.02.019.

Hoste, H., Jackson, F., Athanasiadou, S., Thamsborg, S.M., Hoskin, S.O., 2006. The effects of tannin-rich plants on parasitic nematodes in ruminants. Trends Parasitol. 22 (6), 253-261, S1471-4922(06)00099-7 [pii]. 
Howell, A.B., 2007. Bioactive compounds in cranberries and their role in prevention of urinary tract infections. Mol. Nutr. Food Res. 51 (6), 732737, 10.1002/mnfr.200700038 [doi].

Howell, A.B., Reed, J.D., Krueger, C.G., Winterbottom, R., Cunningham, D.G., Leahy, M., 2005. A-type cranberry proanthocyanidins and uropathogenic bacterial anti-adhesion activity. Phytochemistry 66 (18), 2281-2291, S0031-9422(05)00249-9 [pii].

Katiki, L.M., Ferreira, J.F., Gonzalez, J.M., Zajac, A.M., Lindsay, D.S., Chagas, A.C.S., Amarante, A.F., 2013. Anthelmintic effect of plant extracts containing condensed and hydrolyzable tannins on Caenorhabditis elegans, and their antioxidant capacity. Vet. Parasitol. 192 (1), 218-227.

Klongsiriwet, C., Quijada, J., Williams, A.R., Mueller-Harvey, I., Williamson, E.M., Hoste, H., 2015. Synergistic inhibition of Haemonchus contortus exsheathment by flavonoid monomers and condensed tannins. Int. J. Parasitol. Drugs Drug Resist 5 (3), 127-134, 10.1016/j.ijpddr.2015.06.001 [doi].

Krueger, C.G., Chesmore, N., Chen, X., Parker, J., Khoo, C., Marais, J.P., Shanmuganayagam, D., Crump, P., Reed, J.D., 2016. Critical reevaluation of the 4-(dimethylamino) cinnamaldehyde assay: cranberry proanthocyanidin standard is superior to procyanidin A2 dimer for accurate quantification of proanthocyanidins in cranberry products. Journal of Functional Foods 22, 13-19. 
Krueger, C.G., Reed, J.D., Feliciano, R.P., Howell, A.B., 2013. Quantifying and characterizing proanthocyanidins in cranberries in relation to urinary tract health. Analytical and bioanalytical chemistry 405 (13), 4385-4395.

Krueger, C.G., Dopke, N.C., Treichel, P.M., Folts, J., Reed, J.D., 2000. Matrixassisted laser desorption/ionization time-of-flight mass spectrometry of polygalloyl polyflavan-3-ols in grape seed extract. J. Agric. Food Chem. 48 (5), 1663-1667.

Lange, K.C., Olcott, D.D., Miller, J.E., Mosjidis, J.A., Terrill, T.H., Burke, J.M., Kearney, M.T., 2006. Effect of sericea lespedeza (Lespedeza cuneata) fed as hay, on natural and experimental Haemonchus contortus infections in lambs. Vet. Parasitol. 141 (3-4), 273-278, S0304-4017(06)00346-3 [pii].

Marie-Magdeleine, C., Hoste, H., Mahieu, M., Varo, H., Archimede, H., 2009. In vitro effects of Cucurbita moschata seed extracts on Haemonchus contortus. Vet. Parasitol. 161 (1-2), 99-105, 10.1016/j.vetpar.2008.12.008 [doi].

Max, R.A., 2010. Effect of repeated wattle tannin drenches on worm burdens, faecal egg counts and egg hatchability during naturally acquired nematode infections in sheep and goats. Vet. Parasitol. 169 (1-2), 138-143, 10.1016/j.vetpar.2009.12.022 [doi].

Min, B., Pinchak, W., Anderson, R., Fulford, J., Puchala, R., 2006. Effects of condensed tannins supplementation level on weight gain and in vitro and in vivo bloat precursors in steers grazing winter wheat. J. Anim. Sci. 84 (9), 2546-2554. 
Molan, A.L., Faraj, A.M., 2010. The effects of condensed tannins extracted from different plant species on egg hatching and larval development of Teladorsagia circumcincta (Nematoda: Trichostrongylidae). Folia. Parasitol. (Praha) 57 (1), 62-68.

Monagas, M., Quintanilla-Lopez, J.E., Gomez-Cordoves, C., Bartolome, B., Lebron-Aguilar, R., 2010. MALDI-TOF MS analysis of plant proanthocyanidins. J. Pharm. Biomed. Anal. 51 (2), 358-372, 10.1016/j.jpba.2009.03.035 [doi].

Monteiro, M.V.B., Bevilaqua, C.M.L., Morais, S.M., Machado, L.K.A., CamurçaVasconcelos, A.L.F., Campello, C.C., Ribeiro, W.L.C., Mesquita, M.d.A., 2011. Anthelmintic activity of Jatropha curcas L. seeds on Haemonchus contortus. Vet. Parasitol. 182 (2-4), 259-263, http://dx.doi.org/10.1016/j.vetpar.2011.04.010.

Niezen, J.H., Waghorn, G.C., Graham, T., Carter, J.L., Leathwick, D.M., 2002. The effect of diet fed to lambs on subsequent development of Trichostrongylus colubriformis larvae in vitro and on pasture. Vet. Parasitol. 105 (4), 269-283, S0304401702000250 [pii].

Reed, J.D., Krueger, C. Proanthocyanidin content and structure of fifty-one birdsfoot trefoil strains. Manuscript in preparation.

Reed, J.D., 1995. Nutritional toxicology of tannins and related polyphenols in forage legumes. J. Anim. Sci. 73 (5), 1516-1528.

Shaik, S.A., Terrill, T.H., Miller, J.E., Kouakou, B., Kannan, G., Kaplan, R.M., Burke, J.M., Mosjidis, J.A., 2006. Sericea lespedeza hay as a natural 
deworming agent against gastrointestinal nematode infection in goats. Vet. Parasitol. 139 (1-3), 150-157, S0304-4017(06)00142-7 [pii].

Skantar, A.M., Agama, K., Meyer, S.L., Carta, L.K., Vinyard, B.T., 2005. Effects of geldanamycin on hatching and juvenile motility in Caenorhabditis elegans and Heterodera glycines. J. Chem. Ecol. 31 (10), 2481-2491, 10.1007/s10886-005-7114-z [doi].

Steiner, J., Beuselinck, P., Greene, S., Kamm, J., Kirkbride, J., Roberts, C., 2001. A description and interpretation of the NPGS birdsfoot trefoil core subset collection. Crop Sci. 41 (6), 1968-1980.

Terrill, T.H., Waghorn, G.C., Woolley, D.J., McNabb, W.C., Barry, T.N., 1994. Assay and digestion of 14C-labelled condensed tannins in the gastrointestinal tract of sheep. Br. J. Nutr. 72 (3), 467-477.

USDA, 2014. Germplasm Resources Information Network (GRIN).

Vargas-Magana, J.J., Torres-Acosta, J.F., Aguilar-Caballero, A.J., SandovalCastro, C.A., Hoste, H., Chan-Perez, J.A., 2014. Anthelmintic activity of acetone-water extracts against Haemonchus contortus eggs: interactions between tannins and other plant secondary compounds. Vet. Parasitol. 206 (3-4), 322-327, S0304-4017(14)00535-4 [pii].

Veríssimo, C.J., Niciura, S.C.M., Alberti, A.L.L., Rodrigues, C.F.C., Barbosa, C.M.P., Chiebao, D.P., Cardoso, D., da Silva, G.S., Pereira, J.R., Margatho, L.F.F., da Costa, R.L.D., Nardon, R.F., Ueno, T.E.H., Curci, V.C.L.M., Molento, M.B., 2012. Multidrug and multispecies resistance in sheep 
flocks from São Paulo state, Brazil. Vet. Parasitol. 187 (1-2), 209-216, http://dx.doi.org/10.1016/j.vetpar.2012.01.013.

von Son-de Fernex, E., Alonso-Diaz, M.A., Valles-de la Mora, B., Capetillo-Leal, C.M., 2012. In vitro anthelmintic activity of five tropical legumes on the exsheathment and motility of Haemonchus contortus infective larvae. Exp. Parasitol. 131 (4), 413-418, 10.1016/j.exppara.2012.05.010 [doi]. 


\section{CHAPTER 3}

Anthelmintic efficacy of cranberry vine aqueous and proanthocyanidin extract on ovine Haemonchus contortus

by

Carly D. Barone ${ }^{\mathrm{a}}$, Anne M. Zajac ${ }^{\mathrm{b}}$, Laura A. Manzi-Smith ${ }^{\mathrm{a}}$, Amy B. Howell ${ }^{\mathrm{c}}$, Jess D. Reed ${ }^{\mathrm{d}}$, Christian G. Krueger ${ }^{\mathrm{d}}$, Katherine H. Petersson ${ }^{\mathrm{a}^{*}}$

is prepared for submission to the Veterinary Parasitology journal

\section{Affiliations:}

${ }^{a}$ Department of Fisheries, Animal, and Veterinary Sciences, University of Rhode Island, 120 Flagg Road, CBLS Rm 177, Kingston, Rhode Island, 02881, USA

${ }^{\mathrm{b}}$ Biomedical Sciences and Pathobiology, VA-MD Regional College of Veterinary Medicine, Virginia Tech, Center for Molecular Medicine and Infectious Diseases, 1410 Prices Fork Road, Blacksburg, VA, 24061 USA. ${ }^{c}$ Rutgers, the State University of New Jersey, Philip E. Marucci Center for Blueberry and Cranberry Research, 125a Lake Oswego, Chatsworth, NJ, 08019, USA.

d Animal Science, University of Wisconsin, Madison, 1675 Observatory Drive, Rm 1146, Madison, Wisconsin, 53706, USA. 


\section{Abstract}

The discovery that some forages containing condensed tannins, also called proanthocyanidins (PAC), suppress gastrointestinal nematode (GIN) infection has provided promise for alternative methods of GIN control in small ruminants. This investigation is the first to examine the anthelmintic potential of cranberry vine against the GIN Haemonchus contortus, while advancing the study of plants containing PAC. The purpose of this study was to explore the anti-parasitic potential of cranberry vine proanthocyanidin extract (CV-PAC) and cranberry vine aqueous extract (CV-AqE) on in vitro egg hatching, larval and adult worm mortality, and exsheathment of $H$. contortus, to observe cranberry vine treated $H$. contortus via scanning electron microscopy, and to investigate the efficacy of cranberry vine powder (CV) drench against an experimental infection of $H$. contortus in lambs. The in vitro anthelmintic effect on egg hatching and mortality of L1, L3, and adult worms was determined after 24-hour exposure to varying concentrations of CV-PAC and CV-AqE. The in vivo anthelmintic effect on an experimental infection in lambs was determined by administering $21.1 \mathrm{~g} \mathrm{CV}$ or not to lambs ( $n=9$ per group) for three consecutive days, and collecting fecal egg count (FEC) data for four weeks post treatment. Inhibition of egg hatch was observed in concentrations $\geq 5 \mathrm{mg} / \mathrm{mL} \mathrm{CV-PAC}$ and significant L1 mortality following hatching was observed at concentrations $\geq 0.3 \mathrm{mg} / \mathrm{mL}$. Egg hatch inhibition occurred at $\geq 5 \mathrm{mg} / \mathrm{mL} \mathrm{CV}-\mathrm{AqE}(0.6 \mathrm{mg} \mathrm{PAC} / \mathrm{mL})$, and significant L1 mortality occurred in $\mathrm{L} 1$ exposed to $\geq 1.25 \mathrm{mg} / \mathrm{mL}(0.15 \mathrm{mg} \mathrm{PAC} / \mathrm{mL})$. Third stage larvae exhibited mortality when exposed to $\geq 2.5 \mathrm{mg} / \mathrm{mL}$ CV-PAC; no significant mortality was observed in CV-AqE. Inhibition of L3 exsheathment was observed at 
concentrations $\geq 5 \mathrm{mg} / \mathrm{mL} \mathrm{CV-PAC} \mathrm{but} \mathrm{was} \mathrm{not} \mathrm{observed} \mathrm{at} \mathrm{any} \mathrm{concentration} \mathrm{of} \mathrm{CV-}$ AqE. Adult worm mortality was observed at concentrations $\geq 0.6 \mathrm{mg} / \mathrm{mL} \mathrm{CV-PAC}$ and $\geq 12.5 \mathrm{mg} / \mathrm{mL}$ CV-AqE (1.5 mg PAC/mL). Scanning electron microscopy revealed an accumulation of aggregate on the cuticle in the buccal area of adult worms incubated in both CV-AqE and CV-PAC. Both CV-PAC and CV-AqE demonstrated anti-parasitic effects on all tested stages of $H$. contortus, but the extracts differed in their level of efficacy. In the lamb trial, no significant difference emerged between the weekly average FEC of the groups, but a significant treatment over time effect was observed, in which the groups behaved differently over the course of the trial, possibly indicative of a change in the infection rate of the lambs drenched with CV compared to the control group. These observed differences both in vitro and in vivo suggest a potential effect of $\mathrm{CV}$ against $H$. contortus and support the need for additional in vivo testing to provide further information of the anthelmintic efficacy of $\mathrm{CV}$ for small ruminants.

\section{Introduction}

Gastrointestinal nematodes (GIN) are a major health concern for small ruminant producers, causing anemia, poor body condition, diarrhea, and death. The barber pole worm (Haemonchus contortus) is the most pathogenic GIN species infecting sheep and goats worldwide (Veríssimo et al., 2012). An infected animal sheds eggs onto pasture through defecation, which hatch into L1, develop to L2 and L3 larvae. Infective L3 larvae migrate out of the feces and are ingested by a grazing animal. In the rumen, L3 larvae undergo exsheathment and then develop into L4 and eventually adult worms that reside in the abomasum, where they feed on the blood of 
the host, mate, and produce eggs. With the growth of parasite resistance to commercial dewormers (Jackson and Coop, 2000), alternative methods have been investigated for their efficacy in GIN control. Plants rich in condensed tannins have demonstrated anthelmintic efficacy against different species of GIN (Hoste et al., 2006; Werne et al., 2013, Costa-Júnior et al, 2014).

Condensed tannins, also called proanthocyanidins (PAC), along with a multitude of other secondary plant comounds, have been investigated for their anthelmintic potential. Feeding small ruminants PAC-containing forages, such as sericea lespedeza (Lespedeza cuneata) (Lange et al., 2006; Shaik et al., 2006; Terrill et al., 2009; Acharya et al., 2015), birdsfoot trefoil (Lotus corniculatus) (Heckendorn et al., 2007), sainfoin (Onobrychis viciifolia) (Heckendorn et al., 2007; Desrues et al., 2016), and sulla (Hedysarum coronarium) (Niezen et al., 1998) has been found to show activity against GIN. In vitro testing, using plant derived PAC organic extracts, has demonstrated inhibition of $H$. contortus egg hatch (Vargas-Magaña et al., 2014) and Teladorsagia circumcincta larval development (Molan and Faraj, 2010). Investigators attributed antiparasitic activity of plants against eggs, larvae, and adult worms to water-soluble secondary compounds such as alkaloids, saponins, and phenolic compounds, including proanthocyanidins and hydrolysable tannins (Adamu et al., 2010; Ferreira et al., 2013). Ingestion of PAC-containing forages and in vitro work using crude aqueous extracts as well as organic extracts of plants have all shown anthelmintic efficacy against small ruminant GIN species.

The American cranberry (Vaccinium macrocarpon), native to the United States, is grown predominantly in Wisconsin, Massachusetts, New Jersey, Oregon and 
Washington (Polashock et al., 2014). Cranberries contain an abundance of PAC that has been shown to support urinary tract health through its bacterial anti-adhesion activity (Howell et al., 2005). Not just the fruit, but also the cranberry vines (stems and leaves) contain high levels of PAC (Ferlemi and Lamari, 2016). The annual pruning of cranberry bogs, as the vines come out of winter dormancy, provides a readily available source of vine. The presence of PAC in cranberry vines $(\mathrm{CV})$, coupled with the abundant availability of this crop in the United States, suggests that cranberry vine may be a viable alternative for the control of gastrointestinal parasites in small ruminants.

This study utilizes in vitro and in vivo methods to investigate the anthelmintic efficacy of cranberry vines against various life stages of $H$. contortus. The specific objectives of this study are to: 1) test the anthelmintic efficacy of cranberry vine purified PAC extract (CV-PAC) and cranberry vine crude aqueous extract (CV-AqE) in vitro against $H$. contortus egg hatching, larval survivability and exsheathment, and adult worms; 2) characterize the effect of CV-PAC and CV-AqE on adult worms via scanning electron microscopy; and 3) test CV powder against an experimental infection of $H$. contortus in lambs.

\section{Materials and methods}

\subsection{Preparation and analysis of cranberry vine powder and extracts}

\subsubsection{Phytochemical analyses}

Analyses on the cranberry vine powder $(\mathrm{CV})$ and cranberry vine powder aqueous extract $(\mathrm{CV}-\mathrm{AqE})$ used in this study were performed at the University of Wisconsin-Madison. Phytochemical analyses included the 4- 
(dimethylamino)cinnamaldehyde (DMAC) method (Krueger et al., 2016; Feliciano et al., 2012a) and the matrix assisted laser desorption/ ionization-time of flight mass spectra (MALDI-TOF MS) method (Krueger et al., 2013; Feliciano et al., 2012b). The concentration of PAC within the $\mathrm{CV}$ powder and within the $\mathrm{CV}-\mathrm{AqE}$ extract was determined by the DMAC method, followed by butanol-HCl analysis, using a cranberry proanthocyanidin (c-PAC) standard to reflect the structural heterogeneity of cranberry vine $\mathrm{PAC}$. The $\mathrm{CV}$ powder (used to make the $\mathrm{CV}-\mathrm{PAC}$ and $\mathrm{CV}-\mathrm{AqE}$ extracts, and used in the in vivo trial) contained $108 \mathrm{mg} \mathrm{PAC/g}$ powder (c-PAC equivalents). The structures of the CV-PAC and CV-AqE extracts were determined by MALDI-TOF mass spectra, while high performance liquid chromatography (HPLC) (de Pascual-Teresa et al., 1998) was used to confirm the presence of other secondary compounds present in the extracts.

\subsubsection{Cranberry vine powder (CV)}

Cranberry vines (stems and leaves) of 'Early Black' cultivar were collected at Rutgers Marucci Center for Blueberry and Cranberry Research, Chatsworth, NJ, USA, in the spring of 2013. The cranberry vines were washed with water in a plastic tub, rinsed, and spread out to dry on trays at $65-70^{\circ} \mathrm{C}$ in enclosed heating cabinets for 16 hours. The vines were then ground in a blender before testing the bioactivity of the powder.

\subsubsection{Cranberry vine powder proanthocyanidin extract (CV-PAC)}

Cranberry vine proanthocyanidins (CV-PAC) were extracted from the dried cranberry vine powder at Rutgers Marucci Center for Blueberry and Cranberry Research, Chatsworth, NJ, USA. Proanthocyanidin extract was isolated from the CV 
using solid-phase chromatography according to the established method for PAC isolation (Howell et al., 2005). Reverse phase (C18) followed by adsorption chromatography (Sephadex LH-20) was used to extract and isolate the total PAC. The recovered PAC was stored at room temperature, in the dark. Electrospray mass spectrometry and MALDI-TOF mass spectrometry were utilized to confirm the presence of A-type linkages (Howell et al., 2005).

\subsubsection{Cranberry vine powder aqueous extract ( $C V-A q E)$}

Stock solution of CV-AqE ( $40 \mathrm{mg} / \mathrm{mL}$ ) was prepared by measuring $1.2 \mathrm{~g}$ of $\mathrm{CV}$ into a $50 \mathrm{~mL}$ Falcon ${ }^{\mathrm{TM}}$ tube and adding room temperature tap water up to a volume of $30 \mathrm{~mL}$. Cranberry vine was soaked in the dark for 24 hours. Solid plant matter was centrifuged ( $200 \mathrm{x}$ g for 5 minutes) to the bottom of the tube and the supernatant was collected and subsequently used the same day in the in vitro assays. Stock solution of $40 \mathrm{mg}$ powder $/ \mathrm{mL}$ was made for the egg hatch and $\mathrm{L} 1$ mortality assay. Stock solution of $50 \mathrm{mg}$ powder $/ \mathrm{mL}$ was prepared in the same way and used in the adult mortality assay. The stock solution was stored at $-20^{\circ} \mathrm{C}$ until phytochemical analysis.

\subsection{Parasitology parameters}

Eggs and larvae of $H$. contortus were prepared from lambs at Virginia Tech and University of Rhode Island with experimental infections (L3 identification $>99 \%$ $H$. contortus). Lambs were dewormed to reduce fecal egg counts to $<50$ eggs per gram (epg) and then orally administered 10,000 H. contortus L3. Viability of L1, L3, and adult $H$. contortus was determined by observing motility for five seconds and determined motile (live) or non-motile (dead) (Skantar et al., 2005; Katiki et al., 
2013). If no movement was observed in five seconds, adults and larvae were considered non-motile (dead).

Blood samples were collected weekly via jugular venipuncture into sterile EDTA Vacutainer ${ }^{\mathrm{TM}}$ tubes (Vacutainer ${ }^{\mathrm{TM}}$; Becton Dickinson, Franklin Lakes, NJ). Packed cell volume (PCV) was determined by microhematocrit centrifugation for three minutes at $35,720 \times \mathrm{g}$. Rectal fecal samples were collected weekly for fecal egg count determination according to the modified McMaster technique with a detection limit of 50 epg (Whitlock, 1948).

\subsection{In vitro assays}

\subsubsection{Egg hatch and L1 mortality assay}

The anthelmintic efficacy of CV-PAC and CV-AqE on hatching of $H$. contortus eggs was determined using previously published procedures (Assis et al., 2003; Marie-Magdeleine et al., 2009). Stock solutions in water of CV-AqE (40 $\mathrm{mg} / \mathrm{mL})$ and CV-PAC (20 mg/mL), prepared fresh prior to each assay, were serially diluted with tap water. Concentrations used were based on previous studies using 1.2 $\mathrm{mg} / \mathrm{mL}$ of PAC extracts (Alonso-Díaz et al., 2008; Brunet et al., 2008), and some studies testing concentrations up to $25 \mathrm{mg} / \mathrm{mL}$ (Kamaraj et al., 2011). Feces were collected rectally from donor lambs experimentally infected with $H$. contortus. Eggs were recovered by running fresh feces in water through a series of sieves $(1 \mathrm{~mm}, 355$ $\mu \mathrm{m}, 150 \mu \mathrm{m}, 38 \mu \mathrm{m}, 25 \mu \mathrm{m})$. Eggs retained on the last two sieves were recovered using flotation with a concentrated salt solution (Fecasol®, Vetoquinol U.S.A., Inc., Fort Worth, Texas) and collected on glass cover slips. Recovered eggs were washed in water prior to use in the assay. Eggs (100 eggs/well, in $100 \mu \mathrm{l}$ water) were placed 
into 24-well flat-bottomed microtitre plates (Corning ${ }^{\mathrm{TM}}$, Falcon ${ }^{\mathrm{TM}}$, Polystyrene Microplates, Corning Life Sciences, Tewksbury, MA, USA). Thiabendazole (TBZ, Thermo Fisher Scientific Inc., Waltham, MA, USA) in dimethyl sulfoxide ( 0.5 $\mu \mathrm{g} / \mathrm{mL}$ ) (DMSO, Fisher BioReagents ${ }^{\mathrm{TM}}$, Thermo Fisher Scientific Inc., Waltham, MA, USA) and water were used as positive and negative controls respectively. Dimethyl sulfoxide $(10 \mu \mathrm{l})$ and water $(890 \mu \mathrm{l})$ were added to each well to increase the volume to $1 \mathrm{~mL}$. Serial dilutions of CV-AqE, CV-PAC, or water were added $(1 \mathrm{~mL})$ to each well for a total volume of $2 \mathrm{~mL}$. Final concentrations in wells were 10, 5, 2.5, 1.2, 0.6, 0.3 $\mathrm{mg} / \mathrm{mL}$ for CV-PAC and 20, 10, 5, 2.5, 1.2, $0.6,0.3 \mathrm{mg} / \mathrm{mL}$ for CV-AqE. Five replicates were run for each set of dilutions and controls. The eggs were incubated at $26^{\circ} \mathrm{C}$ for 24 hours and determined to be hatched or not hatched. Results were expressed as percent hatched. The larvae that hatched from the eggs were quantified and determined to be motile or non-motile. Results were expressed as percent mortality of eggs hatched.

\subsubsection{Exsheathment and L3 mortality assay}

The effect of CV-PAC and CV-AqE on the survival and exsheathment of L3 larvae was determined. Fresh dilutions from the stock solutions of CV-AqE (40 $\mathrm{mg} / \mathrm{mL})$ and the CV-PAC $(20 \mathrm{mg} / \mathrm{mL})$ were made each assay and serially diluted using water. The larvae were incubated in CV-PAC $(10,5,2.5,1.2,0.6,0.3,0$ $\mathrm{mg} / \mathrm{mL}), \mathrm{CV}-\mathrm{AqE}(20,10,5,2.5,1.2,0.6,0.3,0 \mathrm{mg} / \mathrm{mL})$ or a water control for 24 hours at $37^{\circ} \mathrm{C}$. Sheathed L3 $(2,000)$ were added to Earle's Balanced Salt Solution (EBSS, Sigma-Aldrich ${ }^{\circledR}$, Inc., Natick, MA, USA) up to a volume of $1 \mathrm{~mL}$ in a $15 \mathrm{~mL}$ Falcon $^{\mathrm{TM}}$ tube (Corning ${ }^{\mathrm{TM}}$ Falcon ${ }^{\mathrm{TM}} 15 \mathrm{~mL}$ Conical Centrifuge Tubes, Corning Life 
Sciences, Tewksbury, MA, USA). Water control $(1 \mathrm{~mL})$ or treatment $(1 \mathrm{~mL})$ was added for a total volume of $2 \mathrm{~mL}$ in the Falcon ${ }^{\mathrm{TM}}$ tube. After 24 hours incubation, percent mortality was determined and exsheathment was induced following a modification of Conder and Johnson (1996). Falcon ${ }^{\mathrm{TM}}$ tubes were covered with Parafilm M® (Parafilm M ${ }^{\circledR}$, Bemis Company, Inc., Neenah, WI). By placing a glass pipet tip (connected to $\mathrm{CO}_{2}$ ) through the Parafilm $\mathrm{M}{ }^{\circledR}$, larvae were rapidly bubbled with carbon dioxide $\left(\mathrm{CO}_{2}\right)$ for 10 minutes to initiate exsheathment, then capped and placed back into the incubator for 18 hours at $37^{\circ} \mathrm{C}$. After the 18 -hour exsheathment incubation, percent larval mortality was determined based on observing motility of 100 larvae/tube for 5 seconds and motile larvae were determined exsheathed or ensheathed. Results were expressed as percent mortality of all larvae (including exsheathed and ensheathed) and percent motile larvae exsheathed.

\subsubsection{Adult worm mortality assay}

The anthelmintic efficacy of CV-PAC and CV-AqE on adult $H$. contortus was determined using previously published in vitro procedures (Kotze and McClure, 2001). Stock solutions of CV-AqE (50 mg/mL) and the CV-PAC (2.4 mg/mL) were made and serially diluted using water. The adult $H$. contortus worms were collected at a commercial abattoir from the abomasa of two naturally infected goats. The abomasa were tied off at each end, placed in bags, and transported to the laboratory. Each abomasum was opened along the greater curvature. Female H. contortus were collected from the folds of the abomasum within 3 hours of slaughter, and washed in RPMI-1640 (GIBCO® RPMI 1640 Medium, Thermo Fisher Scientific Inc., Waltham, MA, USA). Immediately following this wash, each worm was placed for 1-2 hours 
into holding media, containing $2 \mathrm{~mL}$ RPMI-1640 supplemented with $0.8 \%$ glucose (Dextrose (D-glucose) Anhydrous (Granular Powder/Cerified ACS), Fisher Chemical $^{\mathrm{TM}}$, Thermo Fisher Scientific Inc., Waltham, MA, USA), $2.5 \mu \mathrm{g} / \mathrm{mL}$ amphotericin B (Sigma-Aldrich®, Inc., Natick, MA, USA), $100 \mathrm{U} / \mathrm{mL}$ penicillin and $100 \mu \mathrm{g} / \mathrm{mL}$ streptomycin (Corning ${ }^{\mathrm{TM}}$ cellgro $^{\mathrm{TM}}$ Penicillin-Streptomycin Solution, Corning Life Sciences, Tewksbury, MA, USA) and $10 \mathrm{mM} \mathrm{N}-[2-$ hydroxyethyl0piperazine-N-[4-butanesulfonic acid] (HEPES) buffer (HEPES Buffer, Fisher Chemical ${ }^{\mathrm{TM}}$, Thermo Fisher Scientific Inc., Waltham, MA, USA) at $38^{\circ} \mathrm{C}, 5 \%$ $\mathrm{CO}_{2}$. After the holding period, adult female worms were individually placed in CVPAC (1.2, 0.6, $0.3 \mathrm{mg} / \mathrm{mL} ; n=40$ worms/concentration), CV-AqE $(25,12.5,6.2,3.1$ $\mathrm{mg} / \mathrm{mL} ; n=10$ worms/concentration), RPMI-1640 control ( $n=10$ worms), or TBZ ( $0.05 \mathrm{mg} / \mathrm{mL} ; n=10$ worms) control. The CV-PAC assay was the first assay run prior to the CV-AqE assay; therefore a larger number of worms were collected. Fewer worms were collected in subsequent assays due to low variability between worms. Incubation media for all treatments and controls contained RPMI-1640 supplemented with $0.8 \%$ glucose, $10 \mathrm{mmol}$ HEPES, $0.25 \mu \mathrm{g} / \mathrm{mL}$ amphotericin $\mathrm{B}, 10 \mathrm{U} / \mathrm{mL}$ penicillin, and $10 \mu \mathrm{g} / \mathrm{mL}$ streptomycin. The worms were incubated at $38^{\circ} \mathrm{C}, 5 \% \mathrm{CO}_{2}$ up to 48 hours. Mortality of the adult worms, based on observing motility for five seconds, was monitored at 24-hour intervals. Worms were determined motile (live) or non-motile (dead) (Skantar et al., 2005; Katiki et al., 2013) and results were expressed as percent mortality. 


\subsubsection{Scanning electron microscopy (SEM) processing of adult worms}

Adult $H$. contortus were collected at a commercial abattoir from the abomasa of experimentally infected lambs. Worms (10 per treatment or control) were incubated in CV-PAC (12 mg/mL; 12 or 18 hours), CV-AqE (3.1 or $6.2 \mathrm{mg} / \mathrm{mL} ; 18$ hours) or RPMI control (12 or 18 hours). After incubation in CV-AqE, CV-PAC, or RPMI control, all harvested worms (4 worms per treatment or control) were confirmed live and individually preserved in $2 \%$ glutaraldehyde solution in phosphate buffer $(0.1$ $\mathrm{M}, \mathrm{pH}=7.4$ ) (Glutaraldehyde Solution, Electron Microscopy Sciences, Hatfield, PA, USA) and refrigerated at $4^{\circ} \mathrm{C}$ until analysis. Harvested worms were considered representative and examined by SEM. Worms were processed for SEM at the Morphology Service Laboratory at Virginia-Maryland College of Veterinary Medicine, Virginia Tech. Fixed worms were washed in $0.1 \mathrm{M} \mathrm{Na}$ cacodylate buffer and post-fixed with $2 \% \mathrm{OsO}_{4}$ in $0.1 \mathrm{M} \mathrm{Na}$ cacodylate buffer for one hour. Worms were washed again in Na cacodylate buffer and dehydrated in graded ethanol series $(15 \%, 30 \%, 50 \%, 70 \%, 95 \%, 100 \%)$. Worms were then critically point dried (Ladd Critical Point Dryer, Ladd Research Industries, Inc., Williston, Vermont, USA) and sputter coated with gold (SPI-Module Sputter Coater, Structure Probe, Inc., West Chester, Pennsylvania, USA). Immediately following gold coating, worms were observed with a scanning electron microscope (Carl-Zeiss, EVO, Scanning Electron Microscope, Jena, Germany) at the anterior end and the vulva area. 


\subsection{In vivo assay}

\subsubsection{Study subjects}

This study was conducted with the approval of the Institutional Animal Care and Use Committee (IACUC) of the University of Rhode Island. The lambs used for this study were Dorset and Dorset/Hampshire crossbred lambs born and housed at the University of Rhode Island Peckham Farm, located in Kingston, RI, USA. Spring born lambs were weaned and kept on pasture until approximately five months of age, with a group average fecal egg count (FEC) of $550 \pm 157$ trichostrongylid eggs per gram (epg). Five weeks prior to the start of the study, lambs were brought indoors and remained in indoor housing for the entirety of the study. Lambs were group fed 0.91 $\mathrm{kg} / \mathrm{lamb} /$ day grain and hay diet and allowed free access to water and trace mineralized salt. Lambs were dewormed orally with two anthelmintics; levamisole hydrochloride (8.8 mg/kg BW, Levasole ${ }^{\circledR}$, Schering-Plough Animal Health Corp., Union, NJ) and ivermectin $\left(0.2 \mathrm{mg} / \mathrm{kg}\right.$ BW, Ivomec ${ }^{\circledR}$, Merial Inc., Duluth, GA). Fecal egg counts (FEC) were $<50$ epg (McMaster) post-anthelmintic treatment.

\subsubsection{Study Design}

Eighteen lambs (11 female, 7 male) were orally administered 10,000 infective $H$. contortus L3 larvae each. Once eggs were present in the feces (approximately 35 days), the lambs were assigned to one of two groups balanced for sex and fecal egg count. One group received cranberry vine powder (21.1 g of dried CV/day for 3 consecutive days, $n=9$ lambs) and one group was the control group (no CV, $n=9$ lambs). Lambs in the cranberry treatment group were orally administered a slurry of $21.1 \mathrm{~g}$ of dried CV suspended in $60 \mathrm{~mL}$ water using a $60 \mathrm{~mL}$ syringe. It was estimated 
that administration of $21.1 \mathrm{~g} /$ day of CV (corresponding to $2.3 \mathrm{~g}$ /day of CV-PAC (21.1 $\mathrm{g} \mathrm{CV} \mathrm{x} 108 \mathrm{mg}$ PAC/ g powder) to each lamb would be equivalent to $0.3 \mathrm{mg} / \mathrm{mL} \mathrm{PAC}$ in the rumen (assuming $8 \mathrm{~L}$ of rumen capacity) per day, assuming that the concentration would move through the GI tract at this estimated $0.3 \mathrm{mg}$ PAC/mL stomach contents. This estimated concentration only describes the initial drenching in an estimated $8 \mathrm{~L}$ of stomach contents, as it might have been further diluted when the lambs were fed hay and grain. Fecal egg count and packed cell volume were monitored at day zero and weekly after the administration of CV for four weeks.

\subsection{Statistical Analysis}

Egg hatch, L1 and L3 mortality, exsheathment, and adult worm mortality data were analyzed using an analysis of variance (ANOVA) and means separated with Dunnett's $t$ test using the GLM Procedure in SAS (SAS Institute Inc., Cary, NC). Treatment means were compared with significance defined as $p \leq 0.05$. The concentration of extracts required to prevent $50 \%\left(\mathrm{LC}_{50}\right)$ and $90 \%\left(\mathrm{LC}_{90}\right)$ of egg hatching were calculated using PROBIT procedure in SAS, using a confidence interval of $95 \%$. Fecal egg count data and packed cell volume data from the in vivo lamb study were analyzed using the GLIMMIX Procedure in SAS. This model included terms Treatment, Week, and Treatment*Week. A stepdown Dunnett adjustment for multiple comparisons test was also done for simple effect comparisons of treatment*week least square means by week and by treatment. Fecal egg count and packed cell volume data were analyzed from day 0 of the treatment through the following four weeks post treatment. Significance of least square means was defined as $p \leq 0.05$. 


\section{Results}

\subsection{Phytochemical analyses}

The ground CV used to make the CV-AqE contained $108 \mathrm{mg} \mathrm{PAC/g} \mathrm{CV} \mathrm{(c-}$ PAC equivalents). The $40 \mathrm{mg} / \mathrm{mL}$ stock solution of CV-AqE extract was analyzed using the DMAC method and contained $4.8 \mathrm{mg}$ PAC/mL extract. The $\mathrm{CV}-\mathrm{AqE}$ extract under MALDI-TOF MS revealed that the CV-AqE was similar to the CVPAC, in that they both showed PAC with A-type linkages and similar polymer distributions. However, after HPLC analysis, in addition to PAC, hydroxycinnamic acids and flavonols were detected in the CV-AqE extract.

\subsection{Effect of $C V-P A C$ on egg hatching and L1 mortality, L3 mortality and} exsheathment, and adult worm mortality

The mean percent of eggs hatched and mortality for hatched L1 larvae for CVPAC, TBZ control, and water control are shown in Table 1. Although egg hatch inhibition was not observed above $39 \%$, the estimated $\mathrm{LC}_{50}$ of CV-PAC against egg hatching would be $26.6 \mathrm{mg} / \mathrm{mL}$ (Table 3). The $\mathrm{LC}_{50}$ of CV-PAC against L1 larvae was $0.3 \mathrm{mg} / \mathrm{mL}$ and the $\mathrm{LC}_{90}$ was $1.6 \mathrm{mg} / \mathrm{mL}$ (Table 3). Hatch of eggs incubated in positive control (TBZ) was 100\% inhibited $(p \leq 0.001)$.

The mean percent mortality and percent exsheathment inhibition of L3 larvae in CV-PAC and water control are shown in Table 1. The $\mathrm{LC}_{50}$ of CV-PAC against the survivability of L3 larvae was $4.0 \mathrm{mg} / \mathrm{mL}$ (Table 3). Of the viable larvae, the $\mathrm{LC}_{50}$ of PAC against exsheathment was $4.0 \mathrm{mg} / \mathrm{mL}$ (Table 3). 
Table 1. Effect of cranberry vine proanthocyanidin extract (CV-PAC) on Haemonchus contortus egg hatch and L1 mortality post-hatch ${ }^{1}$, L3 mortality and exsheathment ${ }^{2}$, and adult worm mortality ${ }^{3}$.

\begin{tabular}{|c|c|c|c|c|c|}
\hline \multirow[t]{3}{*}{ Concentration } & Egg Hatch & L1 Mortality $^{1}$ & L3 Mortality ${ }^{2}$ & Exsheathment & Adult Mortality \\
\hline & Inhibition $^{1}$ & & & Inhibition $^{2}$ & $(48 \mathrm{hrs})^{3}$ \\
\hline & \multicolumn{5}{|c|}{ Percent (\%) } \\
\hline TBZ & $100 \pm 0 * *$ & -- & -- & -- & $94 \pm 3 *$ \\
\hline $0.0 \mathrm{mg} \mathrm{PAC} / \mathrm{mL}$ & $9 \pm 1$ & $3 \pm 1$ & $11 \pm 3$ & $2 \pm 1$ & $7 \pm 3$ \\
\hline $0.3 \mathrm{mg} \mathrm{PAC} / \mathrm{mL}^{+}$ & $7 \pm 1$ & $49 \pm 2 * *$ & $24 \pm 4$ & $1 \pm 1$ & $77 \pm 5^{*}$ \\
\hline $0.6 \mathrm{mg} \mathrm{PAC} / \mathrm{mL}^{+}$ & $7 \pm 1$ & $77 \pm 2 * *$ & $18 \pm 5$ & $5 \pm 2$ & $91 \pm 1 *$ \\
\hline $1.2 \mathrm{mg} \mathrm{PAC} / \mathrm{mL}^{+}$ & $10 \pm 2$ & $79 \pm 4^{* *}$ & $14 \pm 3$ & $8 \pm 4$ & $97 \pm 3^{*}$ \\
\hline $2.5 \mathrm{mg} \mathrm{PAC} / \mathrm{mL}$ & $9 \pm 1$ & $98 \pm 1 * *$ & $34 \pm 5^{* *}$ & $23 \pm 9$ & -- \\
\hline $5.0 \mathrm{mg} \mathrm{PAC} / \mathrm{mL}$ & $22 \pm 2 * *$ & $98 \pm 1 * *$ & $41 \pm 4 * *$ & $59 \pm 9 * *$ & -- \\
\hline $10.0 \mathrm{mg} \mathrm{PAC} / \mathrm{mL}$ & $39 \pm 2 * *$ & $98 \pm 1 * *$ & $88 \pm 2 * *$ & $83 \pm 9 * *$ & -- \\
\hline
\end{tabular}

${ }^{1}$ Eggs ( $n=100$ eggs/well x 5 wells per treatment) were exposed to varying concentrations of CV-PAC, water control $(0.0 \mathrm{mg} / \mathrm{mL})$, or thiabendazole (TBZ, $0.5 \mu \mathrm{g} / \mathrm{mL})$ control for 24 hours and determined hatched or not hatched (percent egg hatch inhibition). The eggs that hatched into L1 larvae were determined dead or alive (L1 mortality). ${ }^{2}$ Larvae (L3) were incubated in varying concentrations of CV-PAC or water control $(0.0 \mathrm{mg} / \mathrm{mL})$ for 24 hours. After the 24 -hour incubation, larvae were determined dead or alive (L3 
mortality), and then exposed to $\mathrm{CO}_{2}$ bubbling to initiate exsheathment and incubated for 18 hours. All live larvae were determined ensheathed or exsheathed ( $n=100$ per concentration per treatment) (percent exsheathment inhibition). ${ }^{3}$ Worms were incubated in varying concentrations of CV-PAC $(n=40$ per concentration), RPMI control $(0.0 \mathrm{mg} / \mathrm{mL}, n=10$ per concentration), or thiabendazole (TBZ, $0.05 \mathrm{mg} / \mathrm{mL}$ ) control for 48 hours. Adult worms were determined dead or alive based on observing motility for five seconds (adult mortality). All values are mean \pm SEM.

-- Percentage unavailable; not tested

${ }^{*} p \leq 0.05$ versus negative control $(0.0 \mathrm{mg} / \mathrm{mL})$ within column.

$p \leq 0.001$ versus negative control $(0.0 \mathrm{mg} / \mathrm{mL})$ within column 
The mean percent mortality of adult worms incubated in CV-PAC, TBZ control, and RPMI control for 48 hours is shown in Table 1. Although slow-moving, no mortality was observed at 24 hours in adult worms incubated in CV-PAC, therefore incubation of the worms was continued until 48 hours at which time the $\mathrm{LC}_{50}$ of PAC was $0.21 \mathrm{mg} / \mathrm{mL}$ (Table 3). Mortality of worms incubated in positive control (TBZ) was $94 \%$ at 48 hours ( $p \leq 0.05$ vs RPMI control and $0.3 \mathrm{mg} / \mathrm{mL} \mathrm{CV-PAC).}$

\subsection{Effect of CV-AqE on egg hatching and L1 mortality, L3 mortality and}

exsheathment, and adult worm mortality

The mean percent of eggs hatched and mortality of hatched L1 incubated in

CV-AqE, TBZ control, and water control are shown in Table 2. The $\mathrm{LC}_{50}$ of CV-AqE against egg hatching was $5.3 \mathrm{mg}$ powder $/ \mathrm{mL}(0.6 \mathrm{mg} \mathrm{PAC} / \mathrm{mL})$ (Table 3). Of the eggs that hatched in the $\mathrm{CV}-\mathrm{AqE}$, the $\mathrm{LC}_{50}$ of $\mathrm{CV}-\mathrm{AqE}$ against $\mathrm{L} 1$ larvae was $1.5 \mathrm{mg}$ powder $/ \mathrm{mL}(0.2 \mathrm{mg} \mathrm{PAC} / \mathrm{mL})$ and the $\mathrm{LC}_{90}$ was $3.0 \mathrm{mg} / \mathrm{mL}(0.36 \mathrm{mg} \mathrm{PAC} / \mathrm{mL})$

(Table 3). Hatch of eggs incubated in positive control (TBZ) was $100 \%$ inhibited ( $p \leq$ $0.001)$.

The mean percent mortality and percent exsheathed L3 larvae in CV-AqE and water control are shown in Table 2. Incubation in CV-AqE, at varying concentrations (0.3 - $20 \mathrm{mg} / \mathrm{mL} \mathrm{CV-AqE}$; $0.04-2.38 \mathrm{mg}$ PAC/mL) for 24-hours, did not cause mortality or inhibit exsheathment of L3 larvae.

The mean percent mortality of adult worms incubated in CV-AqE, TBZ control, and RPMI control for 24 hours is shown in Table 2. Significant mortality occurred by 24 hours in worms incubated in CV-AqE, the $\mathrm{LC}_{50}$ of CV-AqE was 3.4 
$\mathrm{mg}$ powder/mL $(0.4 \mathrm{mg} \mathrm{PAC} / \mathrm{mL})$ and the $\mathrm{LC}_{90}$ was $15.1 \mathrm{mg}$ powder $/ \mathrm{mL}(1.8 \mathrm{mg}$ PAC $/ \mathrm{mL}$ ) (Table 3).

The CV-AqE differed compared to CV-PAC in efficacy against different life stages of $H$. contortus in vitro. For the egg hatch and L1 mortality assay, the $\mathrm{LC}_{50}$ of CV-PAC was $26.6 \mathrm{mg} / \mathrm{mL}$ and $0.3 \mathrm{mg} / \mathrm{mL}$ respectively, while the $\mathrm{LC}_{50}$ of $\mathrm{CV}-\mathrm{AqE}$ was $0.6 \mathrm{mg} / \mathrm{mL}(5.3 \mathrm{mg}$ powder $/ \mathrm{mL})$ and $0.2 \mathrm{mg} / \mathrm{mL}(1.5 \mathrm{mg}$ powder $/ \mathrm{mL})$ respectively. The anti-parasitic effect was observed in the CV-AqE egg hatch assays at corresponding concentrations of PAC 44.3-fold lower and in L1 viability assays at concentrations of PAC 1.5-fold lower than those that were efficacious for the purified CV-PAC extract.

\subsection{Observation of adult worms under scanning electron microscopy (SEM)}

Adult worms incubated in CV-AqE and CV-PAC observed using SEM had accumulated deposits of extract on the cuticle around the buccal area (Fig. 1). There was an accumulation of aggregate ranging from light to heavy on the cuticle and around the buccal region of all adult worms incubated in CV-PAC (Fig. 1. D and E). The worm with the heaviest accumulation is shown (12 hour, $12 \mathrm{mg} / \mathrm{mL} \mathrm{CV-PAC \text {, }}$

Fig. 1. D, E, F).

All but one of the four worms incubated in CV-AqE had heavy accumulation of aggregate amassed on the cuticle and around the buccal area at 6.2 and $3.1 \mathrm{mg} / \mathrm{mL}$ (Fig. 1. G, H, I). The remaining worm showed moderate accumulation (18 hour, 3.1 $\mathrm{mg} / \mathrm{mL}$ CV-AqE, images not shown). Control worms incubated in RPMI (Fig. 1. A, B, C) were free of aggregate accumulation and smoother overall than worms incubated in CV-PAC and CV-AqE. All of the RPMI control worms (two 12 hour, 
one depicted in Fig. 1. A, B, C, one image not shown: two 18 hour, images not shown) were similar. A closer view of the cuticle of worms from both of these treatments revealed a buildup of aggregate in the annular furrows of the cuticle (Fig. 1. F and I) that was not present in RPMI control worms (Fig. 1. C). The normal longitudinal cuticular ridges observed on the RPMI control worms (Fig. 1. C) were well structured compared to the longitudinal ridges observed on the treated worms (Fig. 1. F and I) that were less pronounced.

\subsection{Effect of $C V$ on an experimental infection of $H$. contortus in lambs}

The FEC (mean \pm SEM) of the control and treatment groups are shown in Fig.

2. There was an effect of treatment over time $(p=0.04)$, but significance fell out when comparing treatments at each week. There was a slight suppression in the CV group's average FEC in the first two weeks post treatment, but this suppression did not persist at week three. There was no effect of treatment $(p=0.15)$ or time $(p=$ 0.55) on PCV (mean \pm SEM of all animals for the whole study).

\section{Discussion}

This study determined the potential anti-parasitic efficacy of cranberry vines against $H$. contortus by assaying egg hatch, larval viability and exsheathment in vitro, visualizing the effect of $\mathrm{CV}-\mathrm{PAC}$ and $\mathrm{CV}-\mathrm{AqE}$ on adult worms via scanning electron microscopy, and testing CV against an experimental infection of $H$. contortus in lambs. The extracts CV-PAC and CV-AqE differed in their efficacy against different life stages of $H$. contortus in vitro. The higher efficacy of CV-AqE compared to CVPAC, at the same concentrations of PAC indicate that there are other secondary compounds in CV-AqE contributing to the anthelmintic efficacy against eggs and L1 
Table 2. Effect of cranberry vine aqueous extract (CV-AqE) on Haemonchus contortus egg hatch inhibition and L1 mortality post-hatch ${ }^{1}$, L3 mortality and exsheathment inhibition ${ }^{2}$, and adult worm mortality ${ }^{3}$.

\begin{tabular}{|c|c|c|c|c|c|c|}
\hline \multicolumn{2}{|c|}{ Concentration } & Egg Hatch & L1 Mortality ${ }^{1}$ & L3 Mortality ${ }^{2}$ & Exsheathment & Adult Mortality \\
\hline \multirow[b]{2}{*}{ mg Powder/mL } & \multirow[b]{2}{*}{$\mathrm{mg} \mathrm{PAC} / \mathrm{mL}$} & Inhibition $^{1}$ & & & Inhibition $^{2}$ & $(24 \mathrm{hrs})^{3}$ \\
\hline & & \multicolumn{5}{|c|}{ Percent (\%) } \\
\hline TBZ & -- & $100 \pm 0 * *$ & -- & -- & -- & $94 \pm 3^{*}$ \\
\hline 0.0 & 0.0 & $5 \pm 1$ & $5 \pm 1$ & $3 \pm 1$ & $3 \pm 3$ & $10 \pm 10$ \\
\hline 0.3 & 0.04 & $7 \pm 1$ & $5 \pm 1$ & $4 \pm 2$ & $1 \pm 1$ & -- \\
\hline 0.6 & 0.08 & $9 \pm 2$ & $6 \pm 1$ & $0 \pm 0$ & $1 \pm 2$ & -- \\
\hline 1.3 & 0.15 & $7 \pm 1$ & $16 \pm 1^{* *}$ & $6 \pm 2$ & $8 \pm 2$ & -- \\
\hline 2.5 & 0.3 & $8 \pm 1$ & $59 \pm 1 * *$ & $7 \pm 2$ & $15 \pm 4$ & -- \\
\hline 3.1 & 0.38 & -- & -- & -- & -- & $60 \pm 20$ \\
\hline 5.0 & 0.6 & $29 \pm 5 * *$ & $98 \pm 1^{* *}$ & $2 \pm 1$ & $9 \pm 3$ & -- \\
\hline 6.3 & 0.74 & -- & -- & -- & -- & $50 \pm 10$ \\
\hline 10.0 & 1.19 & $77 \pm 4 * *$ & $97 \pm 2 * *$ & $6 \pm 12$ & $11 \pm 4$ & -- \\
\hline 12.5 & 1.5 & -- & -- & -- & -- & $90 \pm 10^{*}$ \\
\hline
\end{tabular}




\begin{tabular}{|c|c|c|c|c|c|c|}
\hline \multicolumn{2}{|c|}{ Concentration } & Egg Hatch & L1 Mortality & L3 Mortality ${ }^{2}$ & Exsheathment & Adult Mortality \\
\hline \multirow[b]{2}{*}{ mg Powder/mL } & \multirow[b]{2}{*}{$\mathrm{mg} \mathrm{PAC} / \mathrm{mL}$} & Inhibition $^{1}$ & & & Inhibition $^{2}$ & $(24 \mathrm{hrs})^{3}$ \\
\hline & & \multicolumn{5}{|c|}{ Percent (\%) } \\
\hline 20.0 & 2.38 & $100 \pm 0 * *$ & $100 \pm 0 * *$ & $11 \pm 3$ & $4 \pm 2$ & -- \\
\hline 25.0 & 3.0 & -- & -- & -- & -- & $100 \pm 0 *$ \\
\hline \multicolumn{7}{|c|}{ 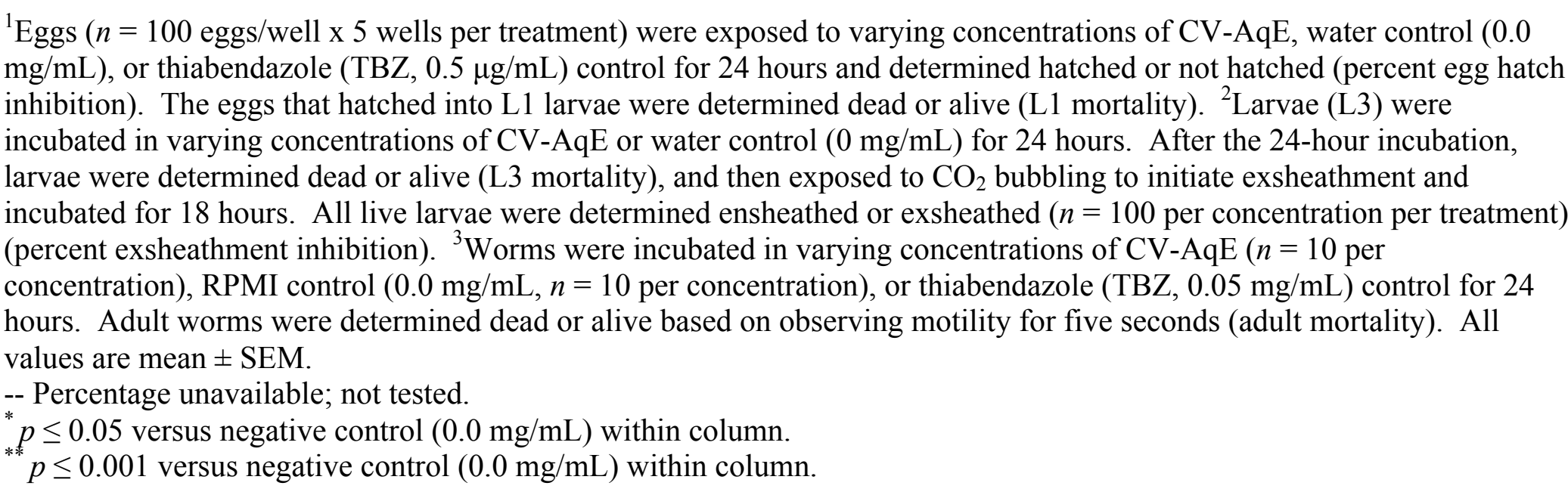 } \\
\hline
\end{tabular}


Table 3. Lethal concentration that killed $50 \%\left(\mathrm{LC}_{50}\right)$ and $90 \%\left(\mathrm{LC}_{90}\right)(\mathrm{mg} / \mathrm{mL})$ and $95 \%$ confidence interval $(95 \% \mathrm{CI})$ of CVPAC and CV-AqE in Haemonchus contortus egg hatch inhibition, L1 mortality, L3 mortality, exsheathment inhibition, and adult worm mortality assays.

\begin{tabular}{|c|c|c|c|c|c|}
\hline & $\begin{array}{l}\text { Egg Hatch } \\
\text { Inhibition }\end{array}$ & L1 Mortality & L3 Mortality & $\begin{array}{l}\text { Exsheathment } \\
\text { Inhibition }\end{array}$ & Adult Mortality \\
\hline \multicolumn{6}{|l|}{$\mathrm{CV}-\mathrm{AqE} \mathrm{LC}_{50}$} \\
\hline mg powder/mL & 5.34 & 1.53 & 137981 & 11143 & 3.37 \\
\hline$(95 \% \mathrm{CI})$ & $(5.03-5.68)$ & $(1.46-1.59)$ & $(1828-2.56 \mathrm{E} 15)$ & $(384.18-4.84 \mathrm{E} 12)$ & $(0.19-5.79)$ \\
\hline $\mathrm{mg} \mathrm{PAC} / \mathrm{mL}$ & 0.64 & 0.18 & 16419 & 1326 & 0.4 \\
\hline$(95 \% \mathrm{CI})$ & $(0.60-0.68)$ & $(0.17-0.19)$ & $(217.5-3.1 \mathrm{E} 14)$ & $(45.7-5.8 \mathrm{E} 11)$ & $(0.02-0.7)$ \\
\hline \multicolumn{6}{|l|}{ CV-AqE LC $\mathrm{C}_{90}$} \\
\hline $\mathrm{mg}$ powder/mL & 19.77 & 2.96 & 460552819 & 10853009 & 15.14 \\
\hline$(95 \% \mathrm{CI})$ & $(17.73-22.34)$ & $(2.77-3.19)$ & $(225678-7.96 \mathrm{E} 26)$ & $(22057-1.50 \mathrm{E} 23)$ & $(8.27-1314.23)$ \\
\hline $\mathrm{mg} \mathrm{PAC} / \mathrm{mL}$ & 2.35 & 0.35 & 54805786 & 1291508 & 1.80 \\
\hline$(95 \% \mathrm{CI})$ & $(2.1-2.66)$ & $(0.33-0.38)$ & $(26856-9.5 \mathrm{E} 25)$ & $(2624.8-1.9 \mathrm{E} 22)$ & $(0.98-156.4)$ \\
\hline \multicolumn{6}{|l|}{ CV-PAC LC LO $_{50}$} \\
\hline $\mathrm{mg} \mathrm{PAC} / \mathrm{mL}$ & 26.64 & 0.30 & 4.02 & 3.97 & 0.21 \\
\hline$(95 \% \mathrm{CI})$ & $(20.95-35.77)$ & $(0.27-0.33)$ & $(3.47-4.76)$ & $(3.16-5.23)$ & $(0.07-0.31)$ \\
\hline \multicolumn{6}{|l|}{ CV-PAC LC 90} \\
\hline $\mathrm{mg} \mathrm{PAC} / \mathrm{mL}$ & 618.64 & 1.58 & 52.26 & 17.79 & 0.75 \\
\hline$(95 \% \mathrm{CI})$ & $(359.17-1221)$ & $(1.43-1.77)$ & $(35.81-84.62)$ & $(11.80-32.86)$ & $(0.55-1.92)$ \\
\hline
\end{tabular}



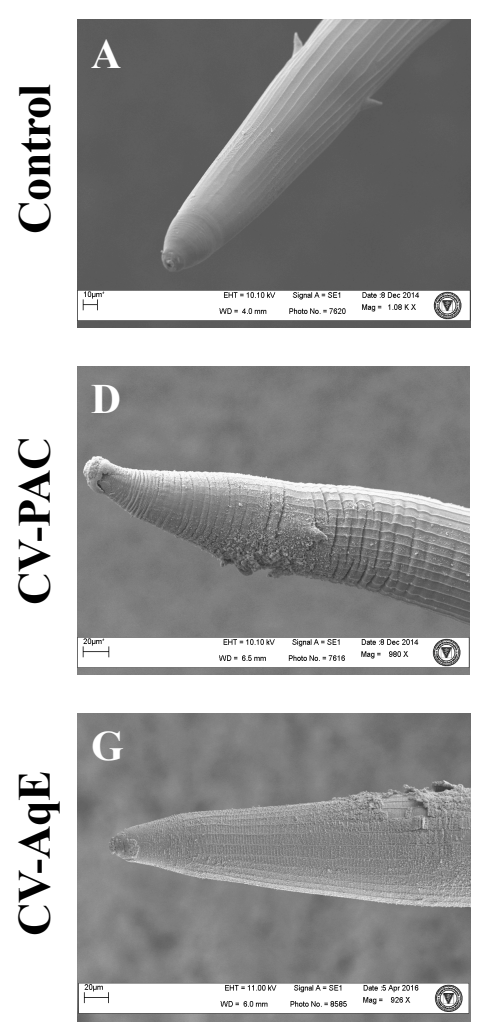
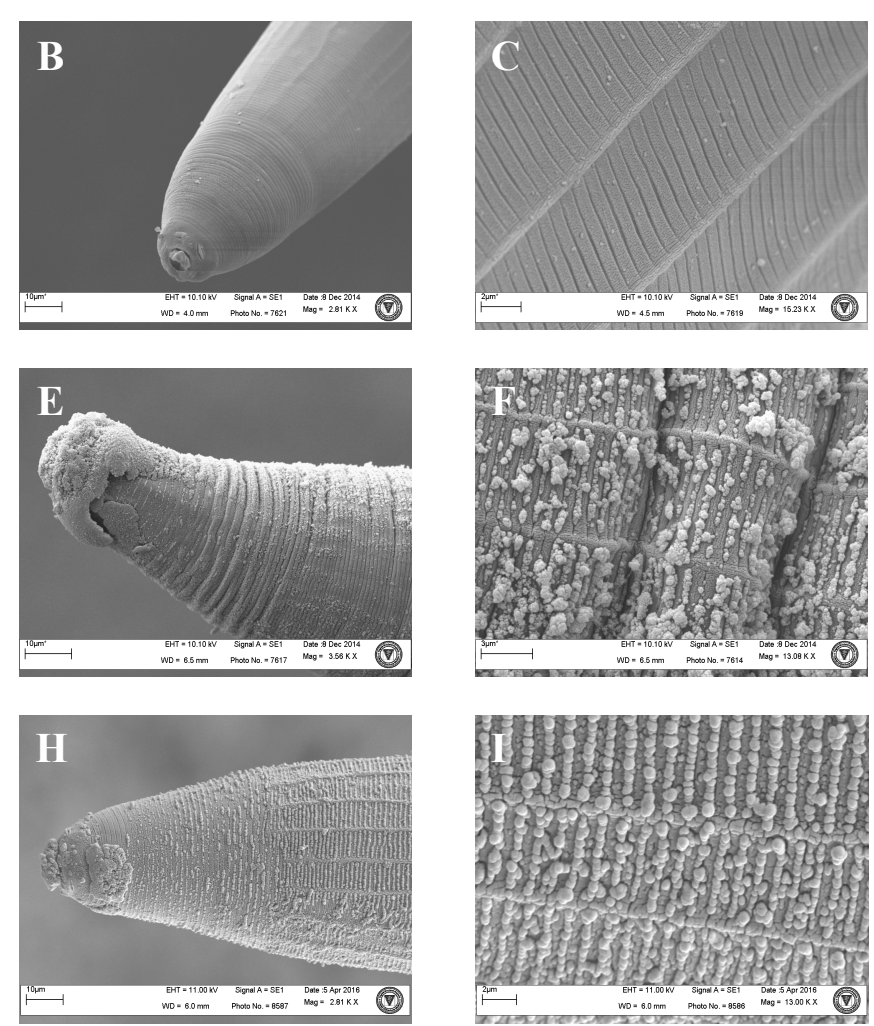

Figure 1. Effect of cranberry vine proanthocyanidin (CV-PAC) and cranberry vine aqueous extract (CV-AqE) on the adult Haemonchus contortus worm through scanning electron microscopy. Scanning electron microscopy of the cephalic end of adult $\mathrm{H}$. contortus maintained in RPMI control (A, B, C; 12 hour), CV-PAC (D, E, F; 12 hour, $12 \mathrm{mg} / \mathrm{mL}$ CV-PAC), or CV-AqE (G, H, I; 18 hour, $3.1 \mathrm{mg} / \mathrm{mL} \mathrm{CV-AqE).} \mathrm{Each} \mathrm{row} \mathrm{depicts} \mathrm{one} \mathrm{worm} \mathrm{from} \mathrm{each}$ treatment, representative of the heaviest accumulation observed. Aggregates of CV-PAC and CV-AqE can be detected around the buccal area $(\mathrm{E}$ and $\mathrm{H})$ as well as on cuticle of the body (F and I). The cuticle of worms that were incubated in RPMI control was compared to worms incubated in CV-PAC and worms incubated in CV-AqE. Accumulation of both CV-PAC and CV-AqE was observed on worms that were incubated in cranberry extracts. 


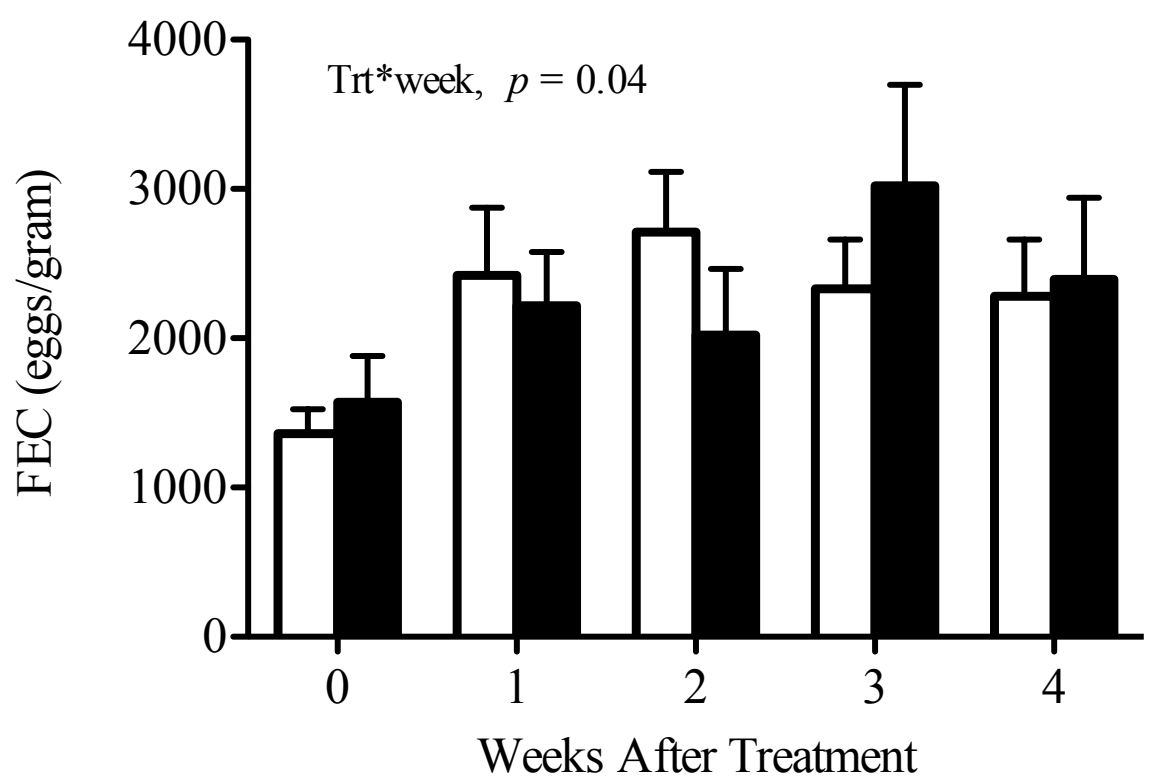

Figure 2. Effect of cranberry vines (CV) on an experimental infection of Haemonchus contortus in lambs. Lambs were experimentally infected with $H$. contortus and infection matured for approximately 35 days. Lambs in treatment group (CV $\mathbf{\square} ; n=9$ ) were orally drenched once daily for 3 consecutive days with 21.1 grams of CV and lambs in control group were not dosed (Control $\square ; n=9$ ). Fecal egg counts (eggs per gram) were monitored for 4 weeks post treatment. Treatment $\mathrm{x}$ week, $p=0.04$, Mean \pm SEM. 
larvae. Alternatively, the extraction method of the purified PAC may have had an effect on the bioactivity of CV-PAC.

Unlike the egg hatch and L1 mortality assay results, the CV-PAC exhibited efficacy against L3 and exsheathment while CV-AqE did not. In order to determine if the extracts were equally efficacious, the solution of CV-AqE would have needed to be more concentrated, $21 \mathrm{mg} / \mathrm{mL} \mathrm{CV-AqE} \mathrm{(2.5} \mathrm{mg/ml} \mathrm{PAC)} \mathrm{and} 42 \mathrm{mg} / \mathrm{mL}(5 \mathrm{mg} / \mathrm{mL}$ PAC). The difference between ovicidal and larvicidal efficacy could also be due to the difference in structural makeup of eggshells and larval cuticles (Riou et al., 2005).

In the adult worm assays, mortality was observed in CV-PAC 24 hours later than mortality recorded for worms incubated in CV-AqE (in comparable PAC concentrations), leading again to the possibility that secondary compounds, other than PAC, could be contributing to the anthelmintic activity observed in the crude CV-AqE extract. Supporting this hypothesis, hydroxycinnamic acids and flavonols were detected in the CV-AqE extract, in addition to PAC. Hydroxycinnamic acids and flavonol are commonly found in high concentrations in cranberry leaves and have been associated with antioxidant acitivity of cranberry leaves (Ferlemi \& Lamari, 2016).

The purified PAC extract used in this study and others (Molan and Faraj, 2010) solely contains PAC with no other impurities. This method includes an organic extraction process and further purifies the material by elution through a Sephadex LH20 column to separate the PAC from the other compounds. Many previously published studies have used crude organic extracts (Hernández-Villegas et al., 2011; Monteiro et al., 2011) investigating PAC containing plants against GIN (Acharya et 
al., 2015; von Son-de Fernex et al., 2012; Klongsiriwet et al., 2015). These investigations have concluded that there are other secondary compounds present in crude organic extracts, besides PAC, which may also be playing a role in the anthelmintic efficacy observed (Hernández-Villegas et al., 2011; Monteiro et al., 2011). The secondary metabolites responsible for the anti-parasitic activity have been identified as saponins, alkaloids, non-protein amino acids, polyphenols (including tannins), lignins and glycosides (Githiori et al., 2006).

In addition to purified PAC extracts and organic extracts, plant extractions using water as a solvent (Adamu et al., 2010; Ferreira et al., 2013) have been extensively investigated against $H$. contortus, attributing antiparasitic activity to alkaloids, saponins (Adamu et al., 2010), and phenolic compounds (Ferreira et al., 2013). Although PAC content was not reported in those studies, these aqueous extracts can contain secondary compounds, including PAC, which can be watersoluble. Studies including Ferreira et al. (2013) used boiling water when creating an aqueous extract, in contrast to the procedure used in this CV investigation, which used room temperature water to infuse the plant matter. The CV was not exposed to boiling water, to eliminate any exposure of the secondary metabolites to high heat, which could cause the compounds to complex (Reed, 1995). The CV-AqE extract was incorporated into this study, rather than an organic extraction, because of its biological relevance to what the animal would actually ingest in an in vivo trial when consuming the ground cranberry vine. The method of grinding and soaking in water would ideally disrupt the cell membrane and cause similar release of PAC as would similarly occur when the animal chews the vine and it is exposed to the neutral stomach juices. 
Previous authors of in vitro trials using the egg hatch assay have reported egg hatch inhibition by PAC (Molan et al., 2002; Molan and Faraj, 2010; Vargas-Magaña et al., 2014) as well as evidence of an anthelmintic effect on the L1 larvae (Molan and Faraj, 2010), consistent with the dose response effect observed in the current study by incubation in varying concentrations of CV-PAC and CV-AqE. In the current study of $\mathrm{CV}$, eggs and larvae were exposed to the extracts for 24 hours. Previous studies have allowed for up to 48 hours exposure to PAC extracts (Vargas-Magaña et al., 2014), but in the current study, eggs were $>90 \%$ hatched by 24 hours. Egg hatch inhibition reported by Molan and Faraj (2010) is comparable to the inhibition observed in this investigation of $\mathrm{CV}$ at $10 \mathrm{mg} / \mathrm{mL}$ CV-PAC (10-fold higher) and $5 \mathrm{mg} \mathrm{CV}$ powder $/ \mathrm{mL}$ CV-AqE (0.6 mg PAC/mL), comparable to $0.9 \mathrm{mg} / \mathrm{mL}$ PAC used by Molan and Faraj (2010). Also, the mortality reported by Molan and Faraj (2010) is comparable to the mortality observed the current study, which landed within the same range of percent inhibition at similar CV-PAC concentrations $(0.3 \mathrm{mg} / \mathrm{mL})$ and lower CV-AqE extract PAC concentrations (1.25 mg CV powder $/ \mathrm{mL} ; 0.15 \mathrm{mg} \mathrm{PAC} / \mathrm{mL})$.

While inhibiting egg hatching may have utility on pasture, the exsheathment process is potentially much more significant. Haemonchus contortus L3 must exsheath in the rumen prior to establishment, therefore inhibition of this stage could have a significant impact on the infection potential of $H$. contortus and thus on the overall health of the animal (Hertzberg et al., 2002; Brunet et al., 2007). The flow rate time of digesta in sheep was investigated, and it was determined that the mean halftime of sheep fed $800 \mathrm{~g}$ lucerne chaff per day using chromium-51 with EDTA marker was $\approx 13.5$ hours in the reticulo-rumen. In sheep fed the same diet, but using cerium- 
144 - praseodymium-144 marker, the mean half-time was $\approx 10$ hours in the reticulorumen (Grovum and Williams, 1977). The L3 H. contortus travel through the gastrointestinal tract with consumed feed and could spend in total, up to $20.1-27$ hours in the reticulo-rumen before exiting. The larvae are able to exsheath during this time in the rumen; therefore, this study incubated the L3 larvae in the CV-PAC and CV-AqE for 24 hours prior to initiating exsheathment. Significant mortality was observed in CV-PAC at 24 hours, and after the initiation of exsheathment, significant inhibition of exsheathment was observed at concentrations $\geq 5 \mathrm{mg} / \mathrm{mL}$ CV-PAC. In contrast, no significant mortality was observed after 24 hours of incubation in the CVAqE, and after the initiation of exsheathment, no significant inhibition in the exsheathment process was observed at any concentration of $\mathrm{CV}$-AqE. A previous study investigating the effect of PAC containing plant extracts on the exsheathment process of $\mathrm{L} 3 \mathrm{H}$. contortus found inhibition of exsheathment in all four plants tested, but did not report the viability of the L3 H. contortus post exsheathment (Alonso-Díaz et al., 2008). Investigators using PAC-rich sainfoin extract observed a dose dependent response, in which exsheathment was delayed significantly at $0.3 \mathrm{mg} / \mathrm{mL}$ and highly inhibited at $1.2 \mathrm{mg} / \mathrm{mL}$, and similar to the previous study, did not report post exsheathment viability percentages of L3 (Brunet et al., 2007). A dose related response was seen in the CV-PAC treatments of this study, but not in the CV-AqE treatments. A potentially important difference that should be noted between these previously published studies and this investigation of $\mathrm{CV}$ is the method of artificial exsheathment. The $\mathrm{CO}_{2}$ exsheathment was considered by Conder and Johnson (1996) to be more reflective of what naturally occurs, therefore in this study, EBSS and 
carbon dioxide was used to investigate the viability of larvae as well as ability to exsheath.

The two extracts, CV-PAC and CV-AqE, were also tested in vitro against adult worms. An investigation of aqueous leaf extract of Annona muricata L. (Annonaceae), which contained phenolic compounds, determined that adult $H$. contortus worms exposed to the extract were immobilized within the first 6-8 hours (Ferreira et al., 2013). This finding agrees with the shorter time to mortality seen for worms incubated in CV-AqE compared to CV-PAC. Although the times observed in the present study are slower than the 6-8 hours to mortality observed by Ferreira et al (2013), this difference could be explained by higher concentrations $(100 \mathrm{mg} / \mathrm{mL})$ used in that study or that control worms lost all motility around 14 hours (Ferreira et al., 2013), compared to this study where worms incubated in control media were $\geq 90 \%$ motile at both 24 and 48 hours.

The mechanism(s) behind the effect of PAC on H. contortus are unknown. The effects that PAC-rich plants have on gastrointestinal parasites vary with the parasite, its stage of development, and the biochemical characteristics of the plant species (Hoste et al., 2006). The nematode cuticle is proline- and hydroxyproline-rich (Thompson and Geary, 1995). The outermost layer of the cuticle, the epicuticle, is lipid-rich and covered by a glycoprotein-rich surface coat which may bind PAC, resulting in the accumulation of material observed on scanning electron microscopy in our study (Page and Johnstone, 2007; Hoste et al., 2006). The multifunctional cuticle plays a crucial role in locomotion (Page and Johnstone, 2007), which is critical for $H$. contortus feeding. The observations made with scanning electron microscopy in this 
study revealed an accumulation of aggregate around the buccal zone and covering most of the cuticular surface coat, specifically in the annular furrows. Investigators observing the changes in adult $H$. contortus worms after in vitro exposure to PAC-rich (sainfoin and tzalam) extracts, observed similar changes under scanning electron microscopy (Martínez-Ortíz-de-Montellano et al, 2013). Investigators suggested that changes in the cuticle, including aggregated material in the buccal area, might affect worm motility and nutrition. The accumulation of aggregate observed on the cuticle of the adult worms could lead to difficulty moving, possibly putting a strain on the adult worm's ability to move towards mating opportunities or sites to feed (MartínezOrtíz-de-Montellano et al, 2013). Alternatively, it is possible that a lack of mobility was the cause of aggregate accumulation observed on the worms because they were not moving as much already, as it has been stated that exposure to PAC-containing plant extracts caused degradation of muscle cells, observed in transmission electron microscopy micrographs of ensheathed H. contortus larvae (Brunet et al., 2011). The accumulation of extract around the buccal area could interfere with normal feeding processes, leading to malnutrition or starvation of the worm. While Martínez-Ortízde-Montellano et al. (2013) did observe changes in the female vulva in vitro, the vulva areas that were viewed in this study of CV did not differ between treatment and control worms.

The CV-PAC and CV-AqE extracts exhibited deleterious effects on Haemonchus contortus life cycle stages, including eggs, larvae, and adults. When a treatment is effective against multiple life cycle stages, the likelihood that the parasite will survive the treatment and persist as a resistant egg, larvae, or worm is reduced, 
theoretically reducing the probability of parasite resistance manifestation (Ferreira et al., 2013). According to Powers et al. (1982) and the W.A.A.V.P. guidelines (Wood et al., 1995) for evaluating in vitro anthelmintic efficacy of drugs, a product inhibiting egg hatch and larval motility in vitro should be considered an effective anthelmintic agent with $\geq 90 \%$ efficacy and considered moderately effective with $80-90 \%$ efficacy (Powers et al., 1982). This investigation of CV has observed between $88-100 \%$ effectiveness against egg hatching, L1 and L3 viability, and adult worm viability using either CV-PAC or CV-AqE at differing concentrations in vitro. Based on $\mathrm{LC}_{90}$ in concentrations $<20 \mathrm{mg} / \mathrm{mL}$ of CV-PAC, efficacy would be observed against L1 larvae, L3 exsheathment and adult worm survivability. Based on $\mathrm{LC}_{90}$ in concentrations $<20 \mathrm{mg}$ powder $/ \mathrm{mL}(<2.4 \mathrm{mg} \mathrm{PAC} / \mathrm{mL})$ of CV-AqE, efficacy would be observed against egg hatching, L1 larvae, and adult worm survivability. This efficacy was only observed under in vitro conditions.

In order to determine if the efficacy observed in vitro could also be confirmed in vivo, the effect of $\mathrm{CV}$ on an established experimental $H$. contortus infection in lambs was tested using a feeding regime that would ultimately represent administration of a concentration of $0.3 \mathrm{mg} / \mathrm{mL}$ PAC per day in the rumen. For the first two weeks after treatment, the FEC of the CV treatment group were slightly suppressed as compared to the average FEC for the control groups over that time period. Investigators who fed goats sericea lespedeza (SL) hay for seven weeks, saw a significant reduction in FEC in as little as one week following initiation of feeding (Shaik et al., 2006). Other investigators, who also fed SL hay to goats, fed for six weeks and did not see a significant reduction in FEC until day 42 of the trial (Terrill et 
al., 2009). Although the percentage of total PAC within SL and CV was comparable (Terrill et al., 2009), much less CV was fed, resulting in administration of approximately $115.7 \mathrm{~g}$ PAC daily in the SL hay versus $2.3 \mathrm{~g}$ PAC daily in the $\mathrm{CV}$, a 50-fold difference. Additionally, PAC from the SL hay could be structurally different from CV PAC.

The slight suppression in FEC over the first two weeks of the trial could be explained by the administration of $\mathrm{CV}$ and the possibility that the worms were damaged by this treatment. In a study feeding SL to lambs for 49 days (Lange et al., 2006), a notable increase in FEC was observed after SL feeding was stopped. It is speculated that the damaged worms would have had the chance to recover from the effects of the three-day treatment by week three. The trial had no effect on PCV of the lambs. As expected, with no significant difference in FEC at each week, there was no significant difference in PCV between the two groups. These results support the need for investigation of further feeding studies of $\mathrm{CV}$ over a longer period of time.

\section{Conclusions}

This study provided in vitro evidence of anthelmintic efficacy using CV-PAC and CV-AqE extracts. Testing with purified CV-PAC confirmed the anti-parasitic role of proanthocyanidins, while testing with $\mathrm{CV}-\mathrm{AqE}$ supported the hypothesis that other secondary compounds are contributing to anthelmintic efficacy. Morphological changes of worms exposed to CV-AqE and CV-PAC in vitro also supported these findings. This study also provided in vivo evidence of anti-parasitic effect of $\mathrm{CV}$ based on slight suppression in FEC. These findings could be further supported by feeding $\mathrm{CV}$ for an extended period of time; creating a longer exposure time between 
H. contortus and $\mathrm{CV}$, warranting the potential use of $\mathrm{CV}$ as a feed supplement for small ruminant GIN control.

\section{Acknowledgments}

The authors of this paper would like to thank University of Rhode Island's Peckham Farm for the care and housing of the lambs used for study. The authors of this study would like to thank Kathy Lowe at Virginia Tech for her expertise and training in scanning electron microscopy and Dr. Stephen Werre at Virginia Tech for his advice and guidance in the statistical analyses used in this study. This work was supported in part by OREI award no. AWD03605 and USDA NE SARE graduate student grant GNE14-071-27806. 


\section{References}

Acharya, M., Burke, J.M., Coffey, K.P., Kegley, E.B., Miller, J.E., Huff, G.R., Smyth, E., Terrill, T.H., Mosjidis, J.A., Rosenkrans, C., 2015. Changes in hematology, serum biochemistry, and gastrointestinal nematode infection in lambs fed sericea lespedeza with or without dietary sodium molybdate. J. Anim. Sci. 93 (4), 1952-1961, 10.2527/jas.2014-8584 [doi].

Adamu, M., Oshadu, O.D., Ogbaje, C.I., 2010. Anthelminthic efficacy of aqueous extract of Acanthus montanus leaf against strongylid nematodes of small ruminants. Afr. J. Tradit. Complement. Altern. Med. 7 (4), 279-285.

Alonso-Diaz, M.A., Torres-Acosta, J.F., Sandoval-Castro, C.A., Aguilar-Caballero, A.J., Hoste, H., 2008. In vitro larval migration and kinetics of exsheathment of Haemonchus contortus larvae exposed to four tropical tanniniferous plant extracts. Vet. Parasitol. 153 (3-4), 313-319, 10.1016/j.vetpar.2008.01.042 [doi].

Assis, L.M., Bevilaqua, C.M.L., Morais, S.M., Vieira, L.S., Costa, C.T.C., Souza, J.A.L., 2003. Ovicidal and larvicidal activity in vitro of Spigelia anthelmia Linn. extracts on Haemonchus contortus. Vet. Parasitol. 117 (1-2), 43-49, http://dx.doi.org/10.1016/j.vetpar.2003.07.021.

Brunet, S., Aufrere, J., El Babili, F., Fouraste, I., Hoste, H., 2007. The kinetics of exsheathment of infective nematode larvae is disturbed in the presence of a tannin-rich plant extract (sainfoin) both in vitro and in vivo. Parasitology 134 (Pt 9), 1253-1262, S0031182007002533 [pii]. 
Brunet, S., Jackson, F., Hoste, H., 2008. Effects of sainfoin (Onobrychis viciifolia) extract and monomers of condensed tannins on the association of abomasal nematode larvae with fundic explants. Int. J. Parasitol. 38 (7), 783-790, S00207519(07)00392-X [pii].

Brunet, S., Fourquaux, I., Hoste, H., 2011. Ultrastructural changes in the third-stage, infective larvae of ruminant nematodes treated with sainfoin (Onobrychis viciifolia) extract. Parasitol. Int. 60 (4), 419-424.

Conder, G.A., Johnson, S.S., 1996. Viability of infective larvae of Haemonchus contortus, Ostertagia ostertagi, and Trichostrongylus colubriformis following exsheathment by various techniques. J. Parasitol. 82 (1), 100-102.

Costa-Júnior, L.M., Costa, J.S., Lôbo, Í.C.P.D., Soares, A.M.S., Abdala, A.L., Chaves, D.P., Batista, Z.S., Louvandini, H., 2014. Long-term effects of drenches with condensed tannins from Acacia mearnsii on goats naturally infected with gastrointestinal nematodes. Vet. Parasitol. 205 (3-4), 725-729, http://dx.doi.org/10.1016/j.vetpar.2014.07.024.

de Pascual-Teresa, S., Treutter, D., Rivas-Gonzalo, J.C., Santos-Buelga, C., 1998. Analysis of flavanols in beverages by high-performance liquid chromatography with chemical reaction detection. J. Agric. Food Chem. 46 (10), 4209-4213.

Desrues, O., Pena-Espinoza, M., Hansen, T.V., Enemark, H.L., Thamsborg, S.M., 2016. Anti-parasitic activity of pelleted sainfoin (Onobrychis viciifolia) against Ostertagia ostertagi and Cooperia oncophora in calves. Parasit. Vectors 9 (1), 329-016-1617-z, 10.1186/s13071-016-1617-z [doi]. 
Feliciano, R.P., Shea, M.P., Shanmuganayagam, D., Krueger, C.G., Howell, A.B., Reed, J.D., 2012a. Comparison of isolated cranberry (Vaccinium macrocarpon Ait.) proanthocyanidins to catechin and procyanidins A2 and B2 for use as standards in the 4-(dimethylamino) cinnamaldehyde assay. J. Agric. Food Chem. 60 (18), 4578-4585.

Feliciano, R.P., Krueger, C.G., Shanmuganayagam, D., Vestling, M.M., Reed, J.D., 2012b. Deconvolution of matrix-assisted laser desorption/ionization time-offlight mass spectrometry isotope patterns to determine ratios of A-type to Btype interflavan bonds in cranberry proanthocyanidins. Food Chem. 135 (3), 1485-1493, 10.1016/j.foodchem.2012.05.102 [doi].

Ferlemi, A.V., Lamari, F.N., 2016. Berry Leaves: An Alternative Source of Bioactive Natural Products of Nutritional and Medicinal Value. Antioxidants (Basel) 5 (2), 10.3390/antiox5020017, 10.3390/antiox5020017 [doi].

Ferreira, L.E., Castro, P.M., Chagas, A.C., Franca, S.C., Beleboni, R.O., 2013. In vitro anthelmintic activity of aqueous leaf extract of Annona muricata L. (Annonaceae) against Haemonchus contortus from sheep. Exp. Parasitol. 134 (3), 327-332, 10.1016/j.exppara.2013.03.032 [doi].

Githiori, J.B., Athanasiadou, S., Thamsborg, S.M., 2006. Use of plants in novel approaches for control of gastrointestinal helminths in livestock with emphasis on small ruminants. Vet. Parasitol. 139 (4), 308-320, http://dx.doi.org/10.1016/j.vetpar.2006.04.021.

Grovum, W.L., Williams, V.J., 1977. Rate of passage of digesta in sheep. 6. The effect of level of food intake on mathematical predictions of the kinetics of digesta in 
the reticulorumen and intestines. Br. J. Nutr. 38 (3), 425-436, S0007114577000555 [pii].

Heckendorn, F., Haring, D.A., Maurer, V., Senn, M., Hertzberg, H., 2007. Individual administration of three tanniferous forage plants to lambs artificially infected with Haemonchus contortus and Cooperia curticei. Vet. Parasitol. 146 (1-2), 123-134, S0304-4017(07)00052-0 [pii].

Hernández-Villegas, M.M., Borges-Argáez, R., Rodriguez-Vivas, R.I., Torres-Acosta, J.F.J., Méndez-Gonzalez, M., Cáceres-Farfan, M., 2011. Ovicidal and larvicidal activity of the crude extracts from Phytolacca icosandra against Haemonchus contortus. Vet. Parasitol. 179 (1-3), 100-106, http://dx.doi.org/10.1016/j.vetpar.2011.02.019.

Hertzberg, H., Huwyler, U., Kohler, L., Rehbein, S., Wanner, M., 2002. Kinetics of exsheathment of infective ovine and bovine strongylid larvae in vivo and in vitro. Parasitology 125 (Pt 1), 65-70.

Hoste, H., Jackson, F., Athanasiadou, S., Thamsborg, S.M., Hoskin, S.O., 2006. The effects of tannin-rich plants on parasitic nematodes in ruminants. Trends Parasitol. 22 (6), 253-261, S1471-4922(06)00099-7 [pii].

Howell, A.B., Reed, J.D., Krueger, C.G., Winterbottom, R., Cunningham, D.G., Leahy, M., 2005. A-type cranberry proanthocyanidins and uropathogenic bacterial anti-adhesion activity. Phytochemistry 66 (18), 2281-2291, S00319422(05)00249-9 [pii].

Jackson, F., Coop, R.L., 2000. The development of anthelmintic resistance in sheep nematodes. Parasitology 120 Suppl, S95-107. 
Kamaraj, C., Rahuman, A.A., Elango, G., Bagavan, A., Zahir, A.A., 2011. Anthelmintic activity of botanical extracts against sheep gastrointestinal nematodes, Haemonchus contortus. Parasitol. Res. 109 (1), 37-45, 10.1007/s00436-010-2218-y [doi].

Katiki, L.M., Ferreira, J.F., Gonzalez, J.M., Zajac, A.M., Lindsay, D.S., Chagas, A.C.S., Amarante, A.F., 2013. Anthelmintic effect of plant extracts containing condensed and hydrolyzable tannins on Caenorhabditis elegans, and their antioxidant capacity. Vet. Parasitol. 192 (1), 218-227.

Klongsiriwet, C., Quijada, J., Williams, A.R., Mueller-Harvey, I., Williamson, E.M., Hoste, H., 2015. Synergistic inhibition of Haemonchus contortus exsheathment by flavonoid monomers and condensed tannins. Int. J. Parasitol. Drugs Drug Resist 5 (3), 127-134, 10.1016/j.ijpddr.2015.06.001 [doi].

Kotze, A.C., McClure, S.J., 2001. Haemonchus contortus utilises catalase in defence against exogenous hydrogen peroxide in vitro. Int. J. Parasitol. 31 (14), 15631571, S0020751901003034 [pii].

Krueger, C.G., Chesmore, N., Chen, X., Parker, J., Khoo, C., Marais, J.P., Shanmuganayagam, D., Crump, P., Reed, J.D., 2016. Critical reevaluation of the 4-(dimethylamino) cinnamaldehyde assay: cranberry proanthocyanidin standard is superior to procyanidin A2 dimer for accurate quantification of proanthocyanidins in cranberry products. Journal of Functional Foods 22, 1319. 
Krueger, C.G., Reed, J.D., Feliciano, R.P., Howell, A.B., 2013. Quantifying and characterizing proanthocyanidins in cranberries in relation to urinary tract health. Analytical and bioanalytical chemistry 405 (13), 4385-4395.

Lange, K.C., Olcott, D.D., Miller, J.E., Mosjidis, J.A., Terrill, T.H., Burke, J.M., Kearney, M.T., 2006. Effect of sericea lespedeza (Lespedeza cuneata) fed as hay, on natural and experimental Haemonchus contortus infections in lambs. Vet. Parasitol. 141 (3-4), 273-278, S0304-4017(06)00346-3 [pii].

Marie-Magdeleine, C., Hoste, H., Mahieu, M., Varo, H., Archimede, H., 2009. In vitro effects of Cucurbita moschata seed extracts on Haemonchus contortus. Vet. Parasitol. 161 (1-2), 99-105, 10.1016/j.vetpar.2008.12.008 [doi].

Martínez-Ortíz-de-Montellano, C., Arroyo-López, C., Fourquaux, I., Torres-Acosta, J.F.J., Sandoval-Castro, C.A., Hoste, H., 2013. Scanning electron microscopy of Haemonchus contortus exposed to tannin-rich plants under in vivo and in vitro conditions. Exp. Parasitol. 133 (3), 281-286, http://dx.doi.org/10.1016/j.exppara.2012.11.024.

Molan, A.L., Faraj, A.M., 2010. The effects of condensed tannins extracted from different plant species on egg hatching and larval development of Teladorsagia circumcincta (Nematoda: Trichostrongylidae). Folia. Parasitol. (Praha) 57 (1), $62-68$.

Molan, A.L., Waghorn, G.C., McNabb, W.C., 2002. Effect of condensed tannins on egg hatching and larval development of Trichostrongylus colubriformis in vitro. Vet. Rec. 150 (3), 65-69. 
Monteiro, M.V.B., Bevilaqua, C.M.L., Morais, S.M., Machado, L.K.A., CamurçaVasconcelos, A.L.F., Campello, C.C., Ribeiro, W.L.C., Mesquita, M.d.A., 2011. Anthelmintic activity of Jatropha curcas L. seeds on Haemonchus contortus. Vet. Parasitol. 182 (2-4), 259-263, http://dx.doi.org/10.1016/j.vetpar.2011.04.010.

Niezen, J.H., Robertson, H.A., Waghorn, G.C., Charleston, W.A., 1998. Production, faecal egg counts and worm burdens of ewe lambs which grazed six contrasting forages. Vet. Parasitol. 80 (1), 15-27, S0304-4017(98)00202-7 [pii].

Page, A.P., Johnstone, I.L., 2007. The cuticle. WormBook , 1-15, 10.1895/wormbook.1.138.1 [doi].

Polashock, J., Zelzion, E., Fajardo, D., Zalapa, J., Georgi, L., Bhattacharya, D., Vorsa, N., 2014. The American cranberry: first insights into the whole genome of a species adapted to bog habitat. BMC Plant. Biol. 14, 165-2229-14-165, 10.1186/1471-2229-14-165 [doi].

Powers, K.G., Wood, I.B., Eckert, J., Gibson, T., Smith, H.J., 1982. World Association for the Advancement of Veterinary Parasitology (W.A.A.V.P.) guidelines for evaluating the efficacy of anthelmintics in ruminants (bovine and ovine). Vet. Parasitol. 10 (4), 265-284.

Reed, J.D., 1995. Nutritional toxicology of tannins and related polyphenols in forage legumes. J. Anim. Sci. 73 (5), 1516-1528.

Riou, M., Koch, C., Delaleu, B., Berthon, P., Kerboeuf, D., 2005. Immunolocalisation of an $\mathrm{ABC}$ transporter, P-glycoprotein, in the eggshells and cuticles of free- 
living and parasitic stages of Haemonchus contortus. Parasitol. Res. 96 (3), 142-148.

Shaik, S.A., Terrill, T.H., Miller, J.E., Kouakou, B., Kannan, G., Kaplan, R.M., Burke, J.M., Mosjidis, J.A., 2006. Sericea lespedeza hay as a natural deworming agent against gastrointestinal nematode infection in goats. Vet. Parasitol. 139 (1-3), 150-157, S0304-4017(06)00142-7 [pii].

Skantar, A.M., Agama, K., Meyer, S.L., Carta, L.K., Vinyard, B.T., 2005. Effects of geldanamycin on hatching and juvenile motility in Caenorhabditis elegans and Heterodera glycines. J. Chem. Ecol. 31 (10), 2481-2491, 10.1007/s10886-0057114-z [doi].

Terrill, T.H., Dykes, G.S., Shaik, S.A., Miller, J.E., Kouakou, B., Kannan, G., Burke, J.M., Mosjidis, J.A., 2009. Efficacy of sericea lespedeza hay as a natural dewormer in goats: dose titration study. Vet. Parasitol. 163 (1-2), 52-56, 10.1016/j.vetpar.2009.04.022 [doi].

Thompson, D.P. and Geary, T.G. 1995. The structure and function of helminth surfaces, In: Marr, J.J. and Müller, M. (Eds.), Biochemistry and Molecular Biology of Parasites. Academic Press, London, UK pp. 203-232.

Vargas-Magana, J.J., Torres-Acosta, J.F., Aguilar-Caballero, A.J., Sandoval-Castro, C.A., Hoste, H., Chan-Perez, J.A., 2014. Anthelmintic activity of acetonewater extracts against Haemonchus contortus eggs: interactions between tannins and other plant secondary compounds. Vet. Parasitol. 206 (3-4), 322327, S0304-4017(14)00535-4 [pii]. 
Veríssimo, C.J., Niciura, S.C.M., Alberti, A.L.L., Rodrigues, C.F.C., Barbosa, C.M.P., Chiebao, D.P., Cardoso, D., da Silva, G.S., Pereira, J.R., Margatho, L.F.F., da Costa, R.L.D., Nardon, R.F., Ueno, T.E.H., Curci, V.C.L.M., Molento, M.B., 2012. Multidrug and multispecies resistance in sheep flocks from São Paulo state, Brazil. Vet. Parasitol. 187 (1-2), 209-216, http://dx.doi.org/10.1016/j.vetpar.2012.01.013.

von Son-de Fernex, E., Alonso-Diaz, M.A., Valles-de la Mora, B., Capetillo-Leal, C.M., 2012. In vitro anthelmintic activity of five tropical legumes on the exsheathment and motility of Haemonchus contortus infective larvae. Exp. Parasitol. 131 (4), 413-418, 10.1016/j.exppara.2012.05.010 [doi].

Werne, S., Perler, E., Maurer, V., Probst, J.K., Hoste, H., Drewek, A., Heckendorn, F., 2013. Effect of sainfoin (Onobrychis viciifolia) and faba bean (Vicia faba) on the periparturient rise in ewes infected with gastrointestinal nematodes. Small Ruminant Research 113 (2-3), 454-460, http://dx.doi.org/10.1016/j.smallrumres.2013.03.022.

Whitlock, H.V., 1948. Some modifications of the McMaster Helminth egg-counting technique and apparatus. J. Counc. Sci. Res. 21, pp. 177-180.

Wood, I.B., Amaral, N.K., Bairden, K., Duncan, J.L., Kassai, T., Malone, J.B.,Jr, Pankavich, J.A., Reinecke, R.K., Slocombe, O., Taylor, S.M., 1995. World Association for the Advancement of Veterinary Parasitology (W.A.A.V.P.) second edition of guidelines for evaluating the efficacy of anthelmintics in ruminants (bovine, ovine, caprine). Vet. Parasitol. 58 (3), 181-213, 0304401795008062 [pii]. 


\title{
CHAPTER 4
}

Effect of cranberry vine fed to lambs on experimental Haemonchus contortus infections

by

Carly D. Barone ${ }^{\mathrm{a}}$, Anne M. Zajac ${ }^{\mathrm{b}}$, Jess D. Reed ${ }^{\mathrm{c}}$, Christian G. Krueger ${ }^{\mathrm{c}}, \mathrm{K}$. Petersson $^{\mathrm{a}^{*}}$

is prepared for submission to the journal Veterinary Parasitology

\begin{abstract}
Affiliations:
${ }^{a}$ Department of Fisheries, Animal \& Veterinary Science, University of Rhode Island, 120 Flagg Road, CBLS Rm 177, Kingston, Rhode Island, 02881, USA, ${ }^{\mathrm{b}}$ Biomedical Sciences and Pathobiology, VA-MD Regional College of Veterinary Medicine, Virginia Tech, Center for Molecular Medicine and Infectious Diseases, 1410 Prices Fork Road, Blacksburg, VA, 24061, USA, ${ }^{\mathrm{c}}$ Animal Science, University of Wisconsin, Madison, 1675 Observatory Drive, Rm 1146, Madison, Wisconsin, 53706, USA.
\end{abstract}




\section{Abstract}

Condensed tannins, also called proanthocyanidins (PAC), suppress gastrointestinal nematode (GIN) infections in small ruminants. This investigation is part of the examination of the anthelmintic potential of cranberry vine against the GIN Haemonchus contortus. The purpose of this study was to investigate the effect that feeding cranberry vine (CV) to lambs had on 1) an established infection of $H$.

contortus in lambs, 2) the establishment of a trickle infection of $H$. contortus in lambs, and 3 ) the morphology of $H$. contortus adult worms that were exposed to CV in vivo as determined by scanning electron (SEM), transmission electron (TEM), and light microscopy. Lambs were experimentally infected with L3 larvae in two separate trials; 1) CV trial: 21 lambs, stratified into three groups $(n=7)$ based on gender and fecal egg count (FEC) once the infection $(10,000 \mathrm{H}$. contortus) matured; and 2) CVP trial: 14 lambs, stratified into two groups $(n=7)$ based on gender and twin separation, received trickle infection of $500 \mathrm{~L} 3 \mathrm{H}$. contortus 3 times/week for 3 weeks. For the CV trial, lambs were fed CV0 (0 g CV, 250 g chopped alfalfa hay (AH)), CV100 (100 g CV, 150 g AH), or CV200 (200 g CV, 50 g AH). Fecal egg counts (FEC) were measured weekly for the duration of the trial. At the conclusion of the study, total worm burden was determined and five adult female $H$. contortus worms were collected from the abomasum of each lamb and preserved until viewed under SEM, TEM or light microscopy. There was no difference in FEC between CV0 and CV200. Although there was a slight reduction in worm burden in CV200 (3335 \pm 378$)$ versus CV0 (3908 \pm 399$)$, the difference was not significant. Evaluation of worms using SEM showed cracking in the cuticle of the adult worms from the CV200 group, 
compared to minimal or no cracking observed in CV0. Some accumulation of an aggregate was observed on worms collected from the CV200 group. Evaluation of vesicles internal of the microvilli lining of the lumen of the intestine using TEM showed a lower number of vesicles present in the worms collected from the CV200 group. For the CVP trial, lambs were fed CVP0 diet: 0 g CVP, 500 g grain; or CVP200 diet: 500 g CVP, 100 g grain for 8 weeks. Fecal egg counts (FEC) were measured weekly for the duration of the trial. At the conclusion of the study, total worm and larval burden were determined. There was no difference in FEC or total worm burden between CVP0 and CVP200 groups. Results indicate that feeding $200 \mathrm{~g}$ of $\mathrm{CV}$ to lambs experimentally infected with $H$. contortus for five weeks resulted in cuticular damage and possible malnutrition of the adult worms and may have potential as an anti-parasitic against $H$. contortus. Further investigation feeding a higher intake of $\mathrm{CV}$ is warranted.

\section{Introduction}

Gastrointestinal nematodes (GIN) are a health risk for small ruminants that results in economic losses for sheep and goat producers. Of the GIN species commonly infecting small ruminants, the barber pole worm (Haemonchus contortus) is the most pathogenic, causing the majority of production and economic losses (Veríssimo et al., 2012). With the growth in GIN resistance to the available commercial dewormers (Hoste et al., 2006), research has focused on finding alternative methods of GIN control.

Plants containing condensed tannins, specifically proanthocyanidins (PAC), have been shown to suppress GIN infections in vitro, inhibiting egg hatch (Vargas et 
al., 2014) and larval development (Molan and Faraj, 2010). In vivo, feeding sericea lespedeza (Lespedeza cuneata) (Terrill et al., 2009; Acharya et al., 2015), birdsfoot trefoil (Lotus corniculatus) (Heckendorn et al., 2007), sainfoin (Onobrychis viciifolia) (Heckendorn et al., 2007; Desrues et al., 2016), and sulla (Hedysarum coronarium) (Niezen et al., 1998) have shown potential efficacy. In the search for a viable alternative that grows well in the Northeastern United States, and is a feasible economic option for producers, the use of cranberry vines has been investigated (Barone et al., in preparation; Chapter 3). Cranberry vine extracts have shown potential against $H$. contortus eggs, L1/L3 larvae, the exsheathment process, and adult worms in vitro (Barone et al., in preparation; Chapter 3).

The American cranberry (Vaccinium macrocarpon) is a rich sourch of polyphenol compounds, the most abundant being PAC (Feliciano et al., 2016). Native to the United States, cranberries are predominantly grown in Massachusetts, Wisconsin, New Jersey, Oregon, and Washington (Polashock et al., 2014). Both the fruit and the vine of the cranberry plant contain PAC, but this study used cranberry vine, as it is a byproduct of normal pruning operations that is not currently marketed. The objectives of this study were to determine the efficacy of feeding cranberry vine to lambs with an established infection and to determine the efficacy of cranberry vine against the establishment of an infection of $H$. contortus.

\section{Materials and Methods}

2.1. Study subjects

Feeding trials were conducted with the approval of the Institutional Animal Care and Use Committee (IACUC) of the University of Rhode Island (protocol 
number AN1415-015). Dorset and Dorset/Hampshire crossbred lambs born and housed at the University of Rhode Island Peckham Farm, located in Kingston, RI were used for these studies. Spring born lambs were weaned and kept off pasture until approximately five months of age, with a group average fecal egg count (FEC) of $13 \pm$ 5 trichostrongylid eggs per gram (epg) for the lambs in the CV trial, and a group average FEC of $79 \pm 19$ trichostrongylid epg for the lambs in the CVP trial. Lambs were group fed a maintenance ration of $0.91 \mathrm{~kg} / \mathrm{lamb} /$ day grain $(16 \%$ crude protein commercially available pelleted supplement, Central Connecticut Co-op, Manchester, CT, USA), 0.91 kg/lamb/day grass hay (Pleasant View Farms, Somers, CT, USA.), and allowed free access to water and trace mineralized salt. Ten days before the start of the trials, lambs were orally dewormed with: levamisole hydrochloride $(8.8 \mathrm{mg} / \mathrm{kg}$ BW, Levasole ${ }^{\circledR}$, Schering-Plough Animal Health Corp., Union, NJ) and ivermectin $\left(0.2 \mathrm{mg} / \mathrm{kg}\right.$ BW, Ivomec ${ }^{\circledR}$, Merial Inc., Duluth, GA). Fecal egg counts (FEC) were < 50 epg (McMaster) seven days post-anthelmintic treatment.

\subsection{Cranberry vine feeding trials}

Cranberry vine was administered using two different study designs: 1) the CV trial fed chopped CV for 5 weeks against an established infection of $H$. contortus and 2) the CVP trial fed pelleted CV for 8 weeks during administration of a trickle infection of $H$. contortus.

\subsubsection{Feeding trail 1 (CV trial) study design}

Twenty-one lambs (8 female, 13 male) were orally administered 10,000 infective $H$. contortus L3 larvae. Once the infection matured (approximately 35 days), the lambs were assigned to one of three groups balanced for sex, weight, and fecal egg 
count (FEC). The three groups included CV0 $(0 \mathrm{~g} \mathrm{CV}, 250 \mathrm{~g}$ chopped alfalfa hay (AH)), CV100 (100 g CV, 150 g AH), and CV200 (200 g CV, 50 g AH). Fecal egg counts (FEC), packed cell volumes (PCV), and weights were measured at day zero and weekly after the start of the feeding trial. The lambs were offered one of three diets for 5 weeks (CV0 diet: 0 g CV, 250 g chopped alfalfa hay (AH, “Hi Fiber”, Lucerne Farms, Easton, ME, USA); CV100 diet: 100 g CV, 150 g AH; or CV200 diet: 200 g $\mathrm{CV}, 50 \mathrm{~g} \mathrm{AH})$. During the trial, all lambs were group fed $0.91 \mathrm{~kg} / \mathrm{lamb} /$ day grain ( $16 \%$ crude protein commercially available pelleted supplement, Central Connecticut Co-op, Manchester, CT, USA), $0.91 \mathrm{~kg} / \mathrm{lamb} /$ day grass hay (Pleasant View Farms, Somers, CT, USA.), and individually fed one of the three diets (CV0, CV100, or CV200). At the end of the five-week trial, lambs were humanely euthanized and 5 adult female $H$. contortus worms were collected from the abomasum for electron microscopy. Abomasal contents were collected for worm recovery, quantification, and sex and stage of development determination (Fig. 1).

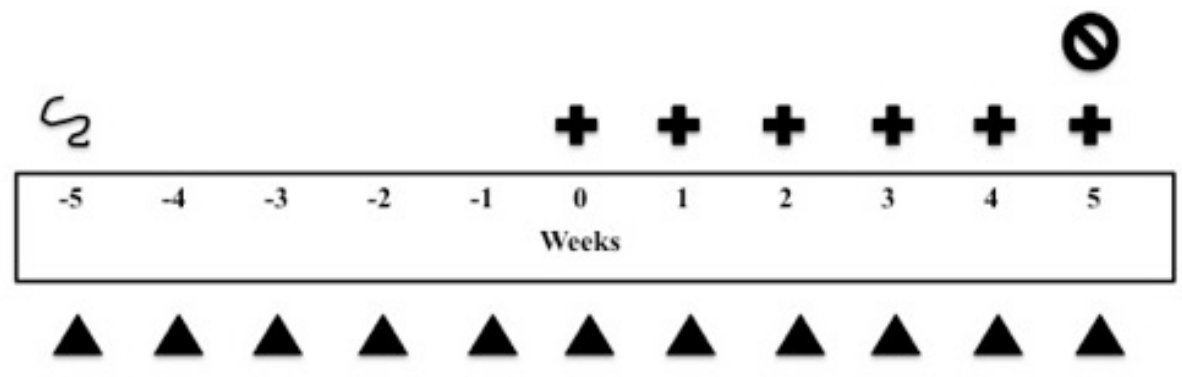

Figure 1. CV Trial: Timeline of trial from -5 to 5 weeks, feeding chopped cranberry vine. Twenty-one lambs were infected with 10,000 L3 H. contortus each at -5 weeks (scribble) once the infection matured (approximately 35 days; day 0) lambs were assigned to one of three groups balanced for sex, weight, and fecal egg count. Lambs were fed hay, grain, and either CV0 diet $(\mathrm{n}=7 ; 0 \mathrm{~g} \mathrm{CV}, 250 \mathrm{~g}$ chopped alfalfa hay $(\mathrm{AH})), \mathrm{CV} 100 \operatorname{diet}(\mathrm{n}=7 ; 100 \mathrm{~g}$ $\mathrm{CV}, 150 \mathrm{~g} \mathrm{AH})$, or CV200 diet ( $\mathrm{n}=7 ; 200 \mathrm{~g} \mathrm{CV}, 50 \mathrm{~g} \mathrm{AH})$ from week 0 to week 5 (\$). Packed cell volume and fecal egg count were processed weekly (triangle). After five weeks of feeding, lambs were sent to the abattoir $(\theta)$, 
where worms were collected for scanning and transmission electron microscopy, and abomasal contents were collected form total worm count.

\subsubsection{Feeding trial 2 (CVP trial) study design}

Seven sets of twin lambs ( 8 male and 6 female) were split into two groups (each group with one of the twins), balanced for sex and weight: CVP0 (500 g grain), or CVP200 (500 g 40\% CV pellet, $100 \mathrm{~g}$ grain). Fourteen days prior (D0) to the experimental infection (D14), the lambs were placed in their groups (4 male and 3 female lambs per group). Lambs were fed either the CVP0 (0 g CVP, 500 g grain) or CVP200 (500 g CVP, 100 g grain) diets for 57 days (from D0 to D56). The extra 100 $\mathrm{g}$ of grain daily given to the lambs consuming the CVP200 diet was done with the purpose to keep the diets balanced for protein and energy. During the trail, all lambs were group fed $0.68 \mathrm{~kg} / \mathrm{lamb} /$ day grain $(16 \%$ crude protein commercially available pelleted supplement, Central Connecticut Co-op, Manchester, CT, USA), 0.91 kg/lamb/day grass hay (Pleasant View Farms, Somers, CT, USA.), and individually fed one of the two diets (CVP0 or CVP200). After feeding CV for two weeks, all lambs were experimentally infected orally (D14) with 500 H. contortus L3 three times a week for three weeks (D14, D16, D18, D21, D23, D25, D28, D30, D32). Fecal egg counts (FEC), packed cell volumes (PCV), and weights were measured at D0 and weekly after the start of the feeding trial for eight weeks. At the end of the eight-week

trial, lambs were sent to the abattoir and abomasal contents were collected for worm recovery, quantification, and identification of developmental stage and sex (Fig. 2). 


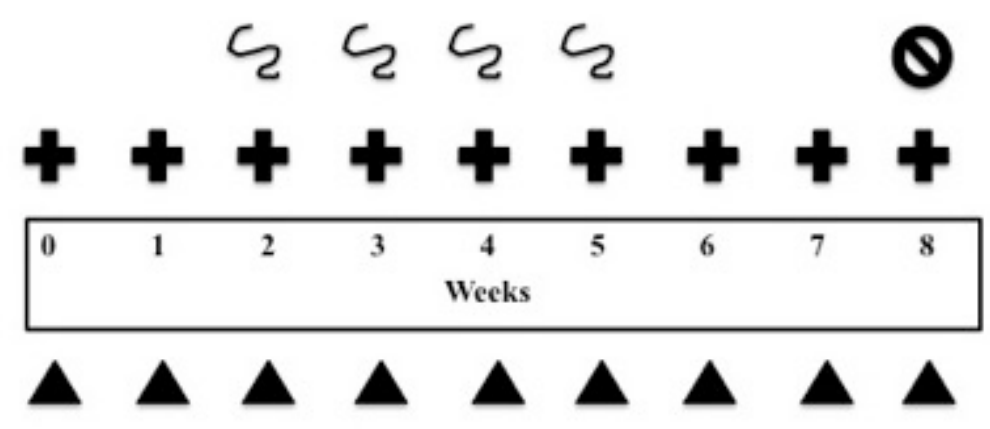

Figure 2. CVP Trial: Timeline of trial from 0 to 8 weeks, feeding pelleted cranberry vine. Fourteen lambs (seven sets of twins) were assigned to one of two groups balanced for sex and weight, each group containing one twin. Lambs were fed either CVP0 diet $(\mathrm{n}=7 ; 0 \mathrm{~g}$ CVP, $500 \mathrm{~g}$ grain) or CVP200 diet ( $\mathrm{n}=7 ; 500 \mathrm{~g}$ CVP, $100 \mathrm{~g}$ grain) from week 0 to week 8 (\$). Each lamb was infected with $500 \mathrm{~L} 3 \mathrm{H}$. contortus 3 times a week, for 3 weeks, spanning between week 2 and week 5 (D14, D16, D18, D21, D23, D25, D28, D30, D32). Packed cell volume and fecal egg count were processed weekly (triangle). After eight weeks of feeding, lambs were sent to the abattoir $(\theta)$, where worms were collected for scanning and transmission electron microscopy, and abomasal contents were collected for total worm count and larval burden.

\subsection{Preparation of Feed}

\subsubsection{Cranberry vines $(\mathrm{CV})$}

Clippings from bogs of 'Stevens' cranberry owned by the A.D. Makepeace

Company (Wareham, MA, USA) were collected following pruning in spring 2015.

Pruned vines were watered until they were placed into burlap bags, transported to the

University of Rhode Island, and air-dried. The collected wet CV (450 kg) yielded 200

$\mathrm{kg}$ of dried $\mathrm{CV}$. The dried vines were chopped through a $3 / 4$ inch screen, using a Gehl

Mix-all Grinder Mixer® (Gehl Compact Equipment, Manitou Americas, Inc., West

Bend, WI, USA) at Cornell University, Ithaca, NY, USA. The chopped cranberry vine was fed as is in the CV trial or fed as a pellet in the CVP trial.

\subsubsection{Cranberry vine pellet (CVP)}


A $40 \%$ CV pellet was manufactured (Central Connecticut Co-op, Manchester, CT, USA) from $\mathrm{CV}$, chopped using a $3 / 4$ inch screen. Main components of the pellet included CV (40\%), red dog flour (17\%), midds-wheat (17\%) and soy (17\%) mixed with molasses (3\%), sheep premix (3\%), and limestone (2\%). The pellet was analyzed for nutritional value to create balanced diets in the CVP trial. Crude protein of the CV pellet was $17.3 \%$ as fed.

\subsubsection{Analysis of cranberry vine proanthocyanidin}

The phytochemical analyses of the cranberry vine (CV) used in this study were performed by the Reed Research Group at the University of Wisconsin-Madison, Madison, WI, USA. Phytochemical analyses included the 4(dimethylamino)cinnamaldehyde (DMAC) method (Krueger et al., 2016; Feliciano et al., 2012a) and the matrix assisted laser desorption/ ionization-time of flight mass spectra (MALDI-TOF MS) method (Krueger et al., 2013; Feliciano et al., 2012b). The concentration of PAC within the CV was determined by the DMAC method, followed by Butanol- $\mathrm{HCl}$ analysis, using a cranberry proanthocyanidin (c-PAC) standard to reflect the structural heterogeneity of cranberry vine PAC. The CV contained $190.6 \mathrm{mg}$ PAC/ g powder or 19.1\% PAC (c-PAC equivalents). The structures of chopped $\mathrm{CV}$ and pelleted $\mathrm{CV}$ were determined by MALDI-TOF mass spectra.

\subsubsection{Analyses of feed}

Random grab samples were collected daily to make weekly composite samples of $\mathrm{CV}, \mathrm{CVP}, \mathrm{AH}$, grain and hay. Weekly composite samples were used to create a 
composite sample for the full duration of the trail. Samples were analyzed by Dairy One Cooperative, Inc. (Ithaca, NY, USA) for crude protein, ADF and NDF.

\subsection{Parasitology parameters}

\subsubsection{Infective larvae}

All third stage (L3) infective larvae used to experimentally infect the lambs were provided by the Zajac parasitology laboratory at the Virginia-Maryland College of Veterinary Medicine, Virginia Tech, Blacksburg, VA, USA. Prior to infection, larvae were confirmed $>90 \%$ viable by observing motility for five seconds and determining motile (dead) or non-motile (alive) (Skantar et al., 2005; Katiki et al., 2013). All larvae were kept at $4^{\circ} \mathrm{C}$ and were less than three months of age when used for infection.

\subsubsection{Blood and fecal analysis}

Blood samples were collected weekly via jugular venipuncture into sterile EDTA Vacutainer tubes (Vacutainer ${ }^{\mathbf{T M}}$; Becton Dickinson, Franklin Lakes, NJ) and packed cell volume (PCV) was determined by microhematocrit centrifugation $(35,720$

$\mathrm{x}$ g) for three minutes. Rectal fecal samples were collected weekly, directly from the rectum, for fecal egg count determination according to the modified McMaster technique (Whitlock, 1948) with a detection limit of 50 epg.

\subsubsection{Analysis of parasite burden}

At the abattoir, the abomasum of each lamb was opened along its greater curvature and contents were collected. Using water, content volume was brought to 2 $\mathrm{L}$, mixed, and a $10 \%$ aliquot was fixed with equal volume of $10 \%$ formalin buffered saline. Total worm burdens were estimated by counting the number of worms in the 
$10 \%$ aliquots, and all counted worms were sexed. The fecundity estimate per female worm was calculated by modification of previously published procedures (Athanasiadou et al., 2001; Gaudin et al., 2016). The average number of eggs per gram recorded on the last day of the trial was determined by running duplicate FEC on two fecal samples, taken at slaughter, per lamb. This FEC was used as the dividend, divided by the total number of female worms at necropsy. Based on the infection method used for the CVP trial, the emptied abomasa were individually soaked overnight in biological saline at $37^{\circ} \mathrm{C}$ to allow immature larvae to migrate out of the abomasal folds. Wash contents were collected for quantification of larval burden. Larval burden was determined by modification of a previously established procedure (Miller, J.E., unpublished protocol). Abomasal wash contents were run through a No. 200 sieve and backwashed with water. Larvae were stained with Lugol's Iodine (Lugol's Iodine, Electron Microscopy Sciences, Hatfield, PA, USA) and sodium hypochlorite was added to allow for counting of larvae using a dissection microscope (Zoom Stereomicroscope, Nikon ${ }^{\circledR}$ SMZ745, MVI, Avon, MA).

\subsubsection{Scanning electron microscopy (SEM) processing of adult worms}

Scanning electron microscopy was only performed on worms from the CV trial. Five live female $H$. contortus worms were collected from the abomasum of each of the lambs at the abattoir. Live worms were individually fixed in $2 \%$ glutaraldehyde solution in phosphate buffer ( $0.1 \mathrm{M}, \mathrm{pH}=7.4)$ (Glutaraldehyde Solution, Electron Microscopy Sciences, Hatfield, PA, USA) and refrigerated at $4{ }^{\circ} \mathrm{C}$ until analysis. Out of the 21 animals processed from the CV trial, two lambs from the CV0 group and five lambs from the CV200 group were investigated using two worms from each of those 
randomly chosen animals. Worms were processed for SEM at the Morphology Service Laboratory at Virginia-Maryland College of Veterinary Medicine, Virginia Tech. Fixed worms were washed in $0.1 \mathrm{M} \mathrm{Na}$ cacodylate buffer and post-fixed with $2 \% \mathrm{OsO}_{4}$ in $0.1 \mathrm{M} \mathrm{Na}$ cacodylate buffer for one hour. Worms were washed again in $\mathrm{Na}$ cacodylate buffer and dehydrated in graded ethanol series $(15 \%, 30 \%, 50 \%, 70 \%$, 95\%, 100\%). Worms were then critically point dried using Ladd Critical Point Dryer (Ladd Critical Point Dryer, Ladd Research Industries, Inc., Williston, Vermont, USA) and placed in the SPI-Module Sputter Coater (SPI-Module Sputter Coater, Structure Probe, Inc., West Chester, Pennsylvania, USA). Immediately following gold coating, worms were observed on a scanning electron microscope (Carl-Zeiss, EVO, Scanning Electron Microscope, Jena, Germany).

\subsubsection{Transmission electron microscopy (TEM)}

Of the individually fixed adult female $H$. contortus worms from the 21 animals from the CV trial processed (5 worms each), two lambs from the CV0 group and five lambs from the CV200 group were investigated using one worm from each of those randomly chosen animals. The worms were processed at the Morphology Service Laboratory at Virginia-Maryland College of Veterinary Medicine, Virginia Tech. Fixed worms were washed in $0.1 \mathrm{M} \mathrm{Na}$ cacodylate buffer and post-fixed with $2 \%$ $\mathrm{OsO}_{4}$ in $0.1 \mathrm{M} \mathrm{Na}$ cacodylate buffer for one hour. Worms were washed again in $\mathrm{Na}$ cacodylate buffer and dehydrated in graded ethanol series $(15 \%, 30 \%, 50 \%, 70 \%$, $95 \%, 100 \%)$. The worms were then set in poly-bed overnight. Once worms were ready to be set in the poly-bed, they were cut at the level of the esophagus and vulva. Both points of interest were placed in position to be thinly cross-sectioned and were 
observed on a transmission electron microscope (JEOL, JEM-1400, Transmission Electron Microscope, Peabody, MA, USA). These worms were also cross-sectioned into thick cuts, placed onto microscope slides, stained with methylene blue, and dried on a hot plate. Thick cut samples were examined using light microscopy ultimately to be used as a reference image for analyzing TEM images.

\subsection{Statistical Analysis}

Worm burden, fecundity, and larval burden data were analyzed statistically using an analysis of variance (ANOVA) and means separated with Dunnett's $t$ test using the GLM Procedure in SAS (SAS Institute Inc., Cary, NC). Treatment means were compared with significance defined as $p \leq 0.05$. Fecal egg count, packed cell volume, and body weight gain data were analyzed using GLM Procedure in SAS with repeated measures analysis of variance and post-hoc test. This model included terms Treatment, Week, and Treatment*Week. Fecal egg count data was analyzed from day zero of the feeding trials through the following five or eight weeks. Least square means significance was defined as $p \leq 0.05$.

\section{Results}

\subsection{Feeding trial 1 (CV trial)}

\subsubsection{Effect of $C V$ on fecal egg count, packed cell volume, and body weight gain}

There was no significant difference in FEC between groups from week zero to week five. At weeks four and five, there did appear to be a slight suppression in the average FEC of the CV200 lambs when compared to control lambs (Fig. 3), but there was no statistical difference. When testing fixed effects across all five weeks, there 


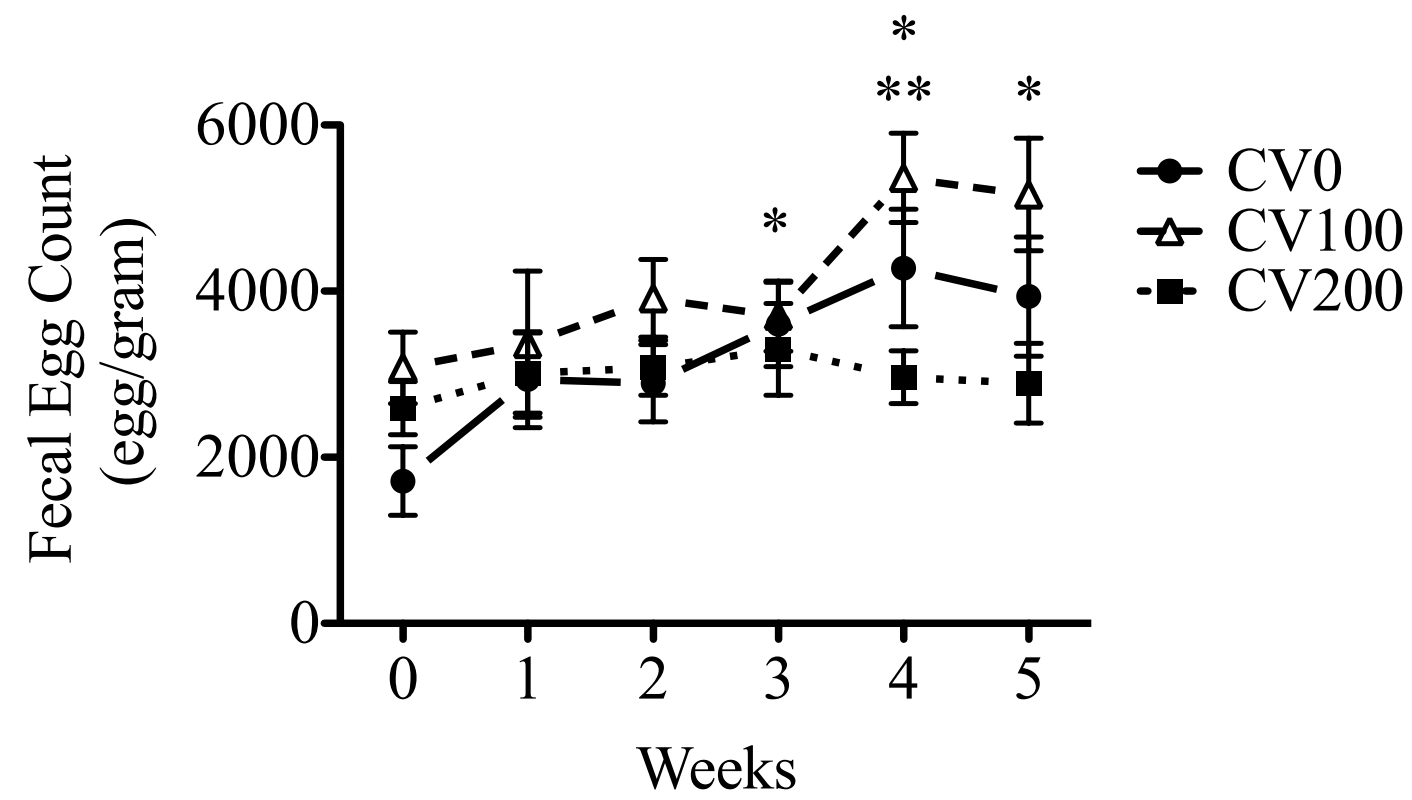

Figure 3. CV Trial: Effect of cranberry vines (CV) on fecal egg counts of an experimental infection of Haemonchus contortus in lambs. Lambs were experimentally infected with $H$. contortus and infection matured for approximately 35 days. Lambs were fed hay, grain, and either CV0 diet ( $0 \mathrm{~g} \mathrm{CV}, 250 \mathrm{~g}$ chopped alfalfa hay (AH)), CV100 diet (100 g CV, 150 g AH), or CV200 diet (200 g CV, 50 g AH), CV200; $\mathrm{n}=$ 7) daily. Fecal egg counts (eggs per gram) were monitored for five weeks. Trt*week, $p$ $=0.04$. Mean \pm SEM.

* weeks 3, 4, 5 CV0 vs week 0 CV0, $p<0.002$

** week 4 CV100 vs week 1 CV100, $p=0.04$

was no difference by treatment $(p=0.12)$ or treatment*week $(p=0.11)$. There was a significant difference by week ( $p<0.0001)$, as the FEC values changed week to week; during week zero through week three, FEC continued to increase and at weeks four and five, while the control and CV100 groups FEC continued increasing, CV200 stayed consistent with its FEC of the previous weeks. Comparing the treatments at each week separately, treatments at weeks zero $(p=0.21)$, one $(p=0.83)$, two $(p=$ $0.36)$ and three $(p=0.86)$ were similar, but at weeks four $(p=0.01)$ and five $(p=$ 0.01) were significantly different. Comparing the FECs over the 5 weeks for each treatment separately, the control group and CV100 group changed significantly over 
the entirety of the five-week trial (control, $p=0.0007$ and CV100, $p=0.0004$ ) while the CV200 group stayed around the same average FEC throughout the five weeks (least square means, $p=0.72$ ).

The mean PCV for CV0 group was $25 \pm 0.4$ (Week 0; $23 \pm 1$, Week 5; $26 \pm 1$ ), the CV100 group averaged $23 \pm 0.2$ (Week 0; $23 \pm 1$, Week 5; $24 \pm 1$ ), and the CV200 group averaged $26 \pm 0.4$ (Week $0 ; 26 \pm 1$, Week 5; $28 \pm 1$ ), over the entire course of the trial. Although there was no significant difference between average PCV at each week, there was a treatment over time effect (Treatment*Week, $p=0.04$ ) as the groups did behave differently, and there was a week effect (Week, $p=0.02$ ) as PCV values did change over the course of the $\mathrm{CV}$ feeding trial as the infection developed, but no treatment effect (Treatment, $p=0.13$ ) was observed (Fig. 4).

Average body weight gain over the 8 -week trial of CV0 ( $83 \pm 71 \mathrm{~g} / \mathrm{day})$, CV100 (124 $\pm 106 \mathrm{~g} /$ day), and CV200 (161 $\pm 123 \mathrm{~g} /$ day) groups was not significantly different when comparing CV200 $(p=0.59)$ and CV100 $(p=0.75)$ to CV0. Although there was no significant difference between weights at each week (Treatment, $p=$ 0.98), there was a treatment over time effect (Treatment*Week, $p=0.05$ ) as the groups behaved differently over time. There was a significant difference by week (Week, $p<0.0001$ ) as the weights increased over the course of the feeding trial because the lambs were growing and gaining weight. When testing the effects for treatment*week (sliced by week), there was no significant difference between the treatments at any week (Week $0, p=0.89$, Week $1, p=0.99$, Week 2, $p=0.99$, Week $3, p=0.99$, Week 4, $p=0.88$, Week 5, $p=0.99$ ) (Figure not shown). 


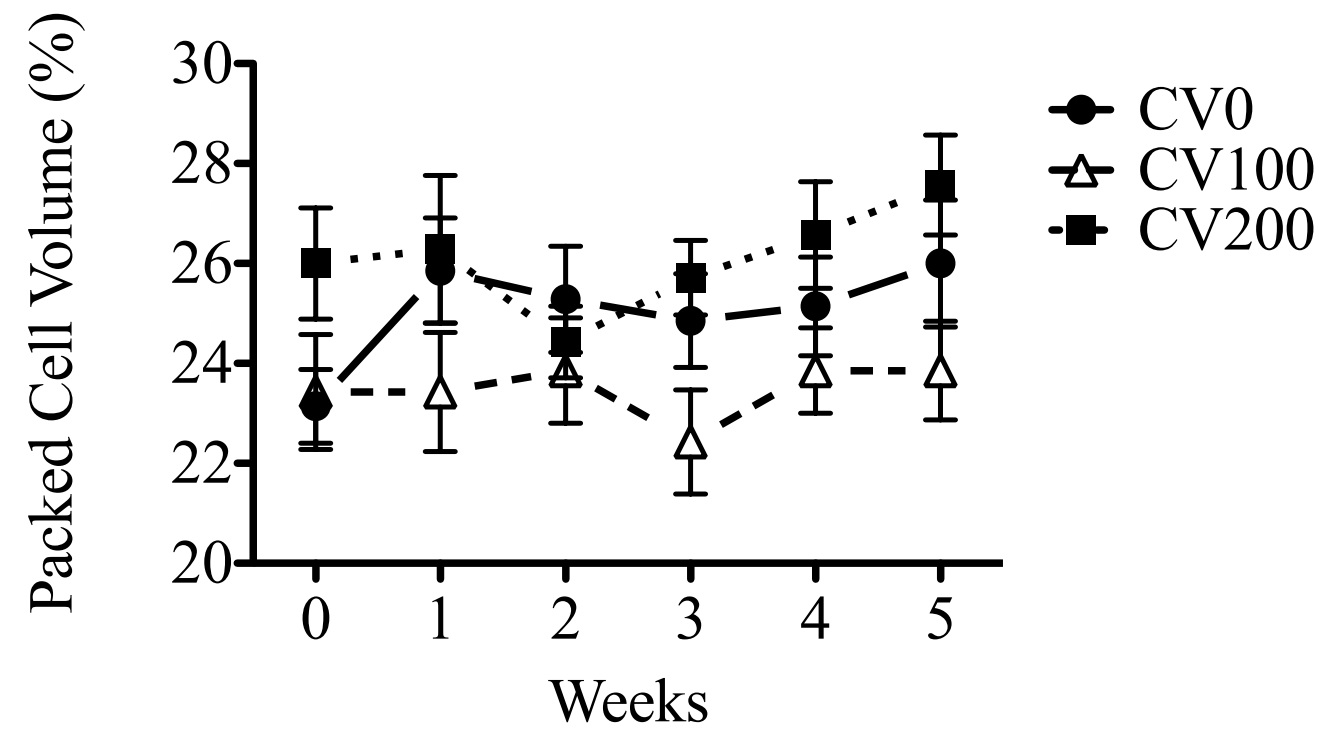

Figure 4. CV Trial: Effect of cranberry vines $(\mathrm{CV})$ on packed cell volume of an experimental infection of Haemonchus contortus in lambs. Lambs were experimentally infected with $H$. contortus and infection matured for approximately 35 days. Lambs were fed hay, grain, and either $\mathrm{CV} 0$ diet $(0 \mathrm{~g} \mathrm{CV}, 250 \mathrm{~g}$ chopped alfalfa hay (AH)), CV100 diet (100 g CV, $150 \mathrm{~g} \mathrm{AH})$, or CV200 diet (200 g CV, $50 \mathrm{~g} \mathrm{AH}), \mathrm{CV} 200 ; \mathrm{n}=7)$ daily. Packed cell volumes $(\%)$ were monitored for five weeks. Trt*week, $p=0.04$. Week, $p=0.02$.

Mean \pm SEM. 

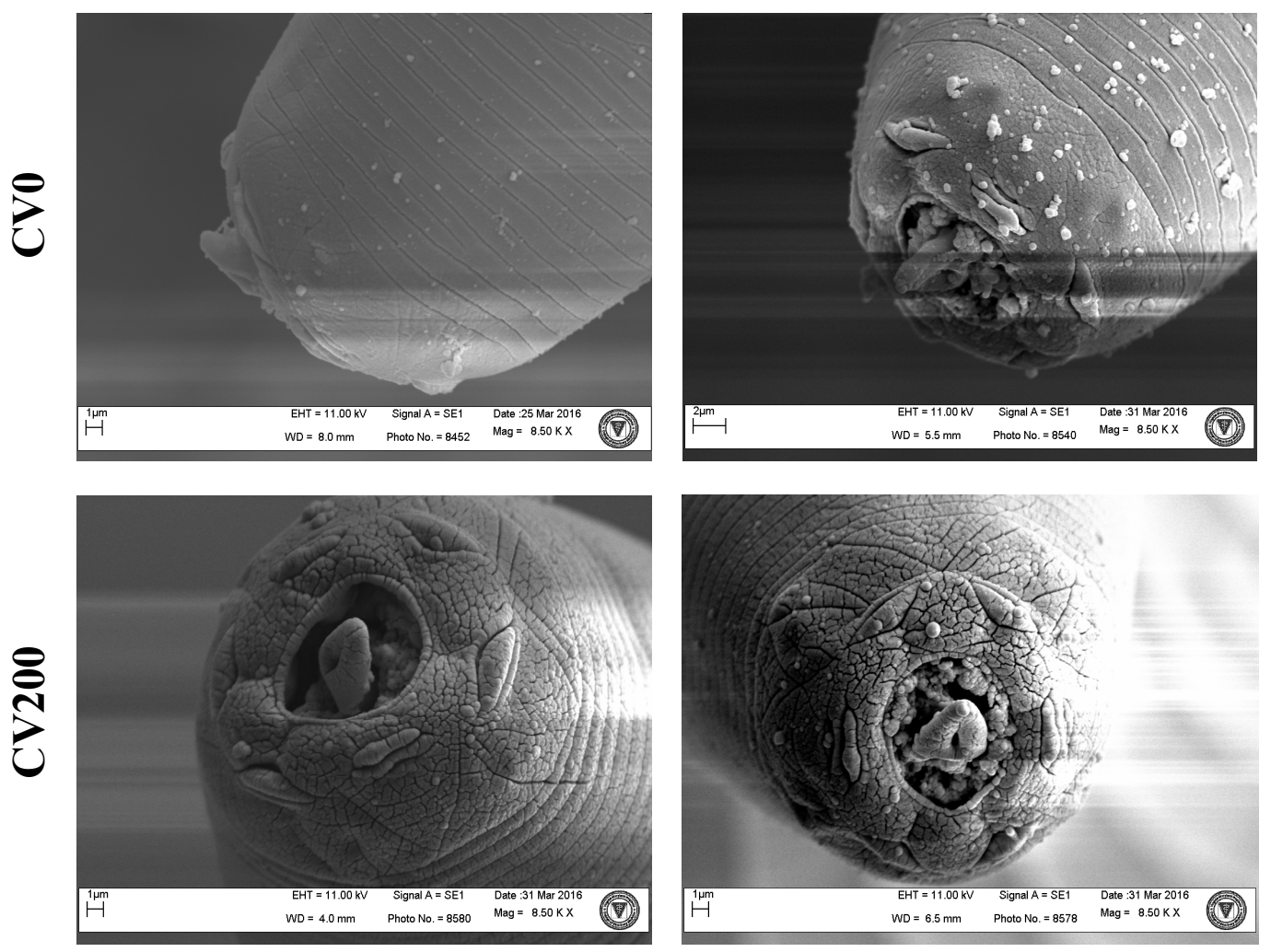

Figure 5. Scanning electron microscopy of Haemonchus contortus worms exposed to control and cranberry vine (CV) diets in vivo. Micrographs of the cephalic end of adult $H$. contortus show CV200 worms with cracking of the cuticle, compared to the control worms (CV0), which had minimal to no cracking of the cuticle.

\subsubsection{Effect of $C V$ on worm burden and fecundity}

There was no difference in the number of worms recovered from the control

(3909 \pm 399$),$ CV100 (4119 $\pm 427, p=0.73$ vs control, $p=0.2$ vs CV200), and CV200

$(3336 \pm 378, p=0.32$ vs control, $p=0.2$ vs CV100) lambs (Figure not shown). The percentage of female worms in the CV0 group was $51 \pm 5$ percent and the CV200 group was $58 \pm 2$ percent. There was no significant difference between the percentages of females of the two groups ( $p=0.19$, Figure not shown). The fecundity value of the CV0 group was $2 \pm 0.2$ and the fecundity value of the CV200 group was $1.8 \pm 0.4(p=0.59)$ (Figure not shown) 


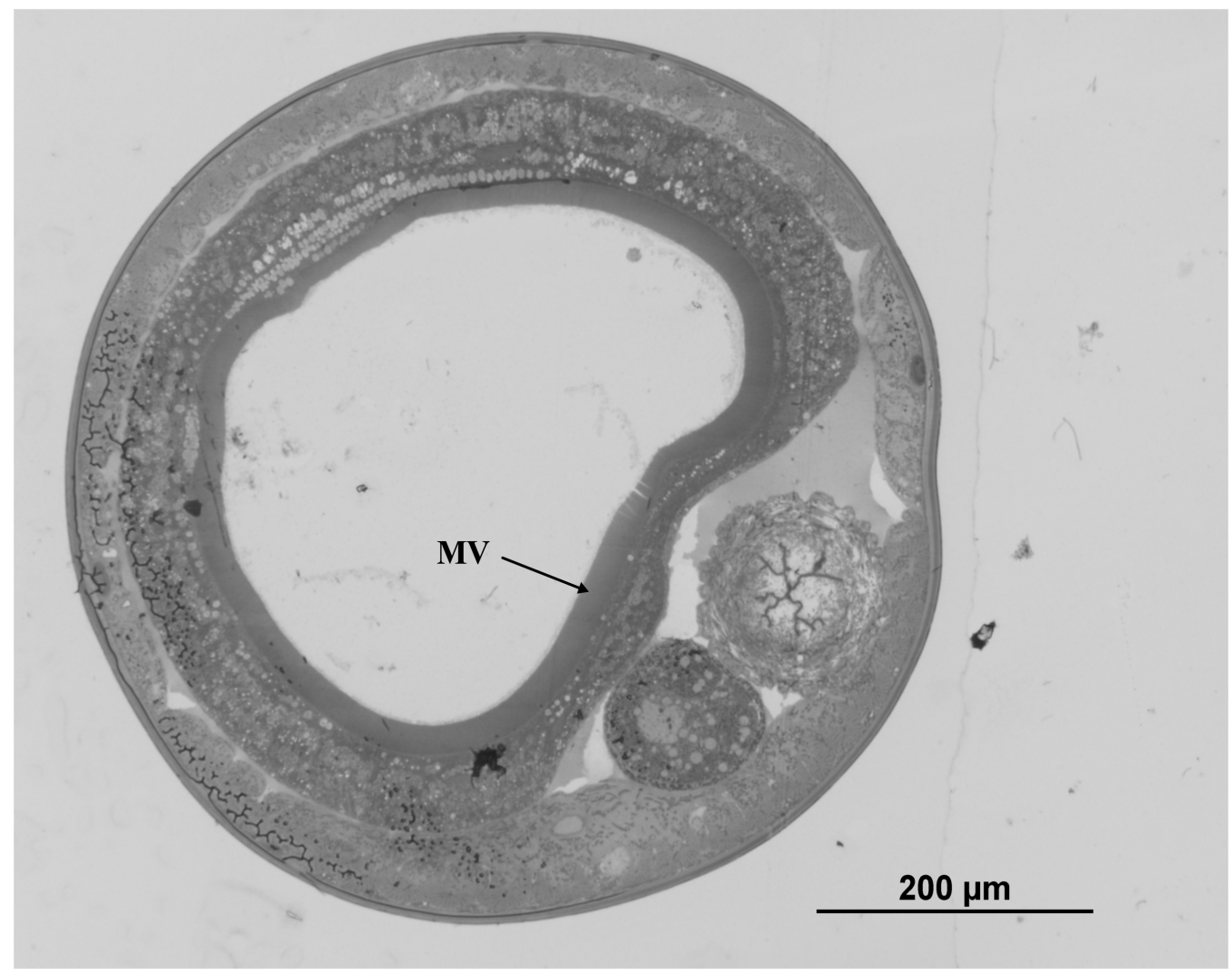

Figure 6. Adult Haemonchus contortus cross-section viewed with light microscopy. Thin cut through the mid section of a female $H$. contortus adult worm, stained and placed on slide, viewed under light microscopy for reference. (MV = microvilli) 

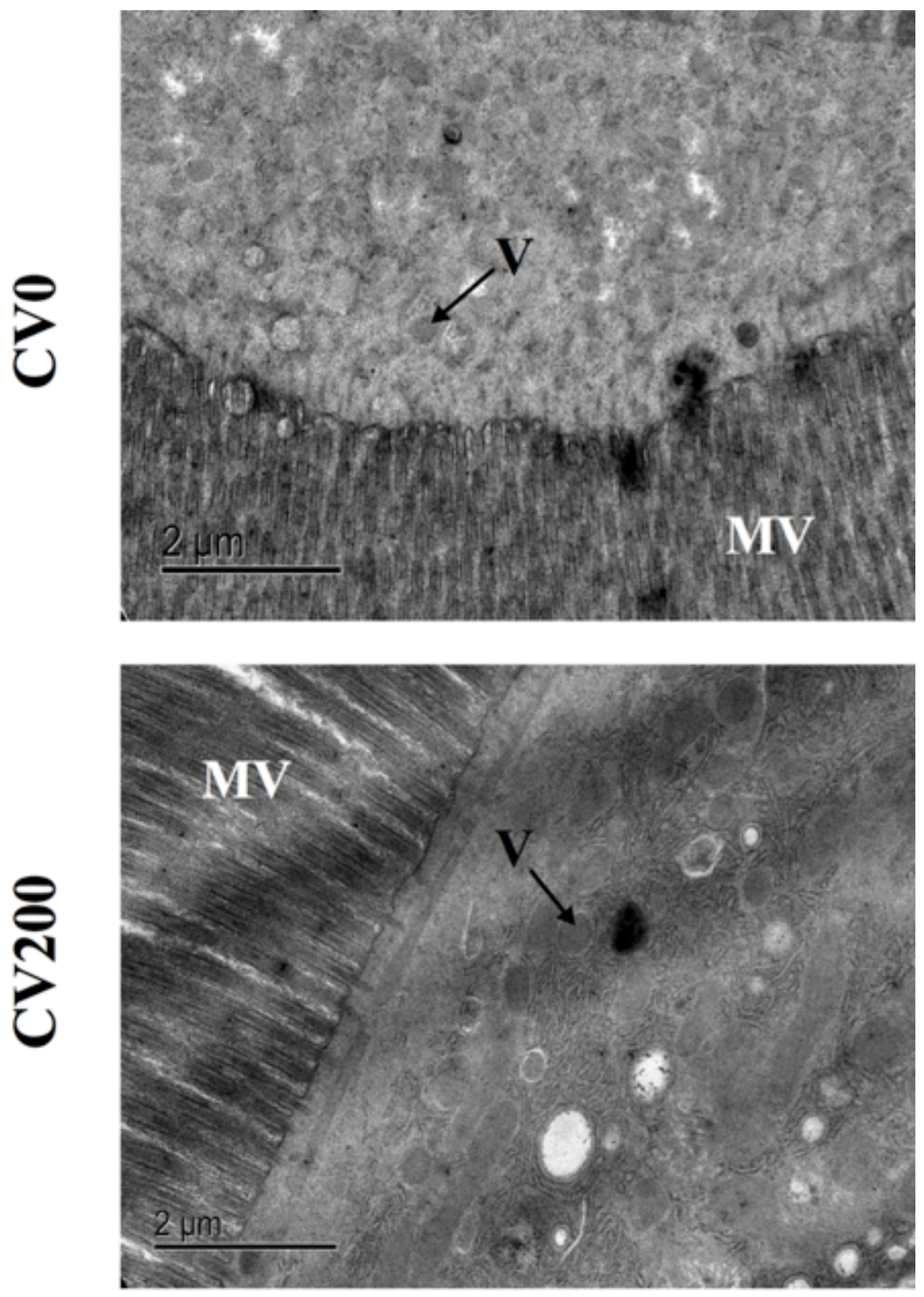

Figure 7. Transmission electron microscopy of Haemonchus contortus worms exposed to control and cranberry vine (CV) diets in vivo. Cross-section micrographs of the microvilli lining the lumen of the intestine of adult $H$. contortus show control worm have more vesicles present internal to the microvilli lining, compared to CV200 worms with a low number of vesicles internal to the microvilli lining. $(\mathrm{MV}=$ microvilli; $\mathrm{V}=$ vesicle $)$ 


\subsubsection{Effect of $C V$ on $H$. contortus worms under SEM}

The worms observed via scanning electron microscopy showed a cracking effect on the cuticle of the worms exposed to CV in vivo (Fig. 5). Although there was some cracking observed on the worms exposed to the CV0 diet, the degree of cracking seen on the CV200 worms was much greater. The worms observed using light microscopy did not show any differences between worms exposed to the CV0 diet and CV200 diet. The light microscopy photos were used as a tool to view the entire crosssection as a reference point (Fig. 6) for the TEM images, which only showed small portions of the cross-sections in a grid.

\subsubsection{Effect of CV on H. contortus worms under TEM}

The worms observed under transmission electron microscopy indicated that the worms exposed to CV200 had lower numbers of vesicles internal to the microvilli lining of the lumen of the intestines (Fig. 7).

\subsection{Feeding trial 2 (CVP trial)}

\subsubsection{Effect of CVP on fecal egg count, packed cell volume, and body weight gain}

In the CVP trial, there was no significant difference between CVP0 and CVP200 FEC at any week. When testing fixed effects across all eight weeks, there was no difference by treatment $(p=0.92)$ or treatment*week $(p=0.99)$ (Fig. 8). There was a significant difference by week $(P<0.0001)$; as both treatments showed significantly increased FEC at weeks six (CVPO, $p=0.003 ;$ CVP200, $p=0.009)$ seven $(p<0.0001)$, and eight $(p<0.0001)$ compared to week zero.

The mean PCV for the CVP0 group over the course of the trial averaged $29 \pm$ 0.3 (Week 0; $29 \pm 1$, Week 8; $30 \pm 0.6$ ) and the mean PCV for the CVP200 group 
averaged $29 \pm 0.3$ (Week $0 ; 30 \pm 1$, Week $8 ; 29 \pm 0.5$ ) over the entire course of the trial. When testing fixed effects across all eight weeks, there was no difference by treatment $(p=0.77)$, but there was a significant difference when testing treatment*week $(p=0.03)$ and a significant difference by week $(P<0.006)$ (Figure not shown).

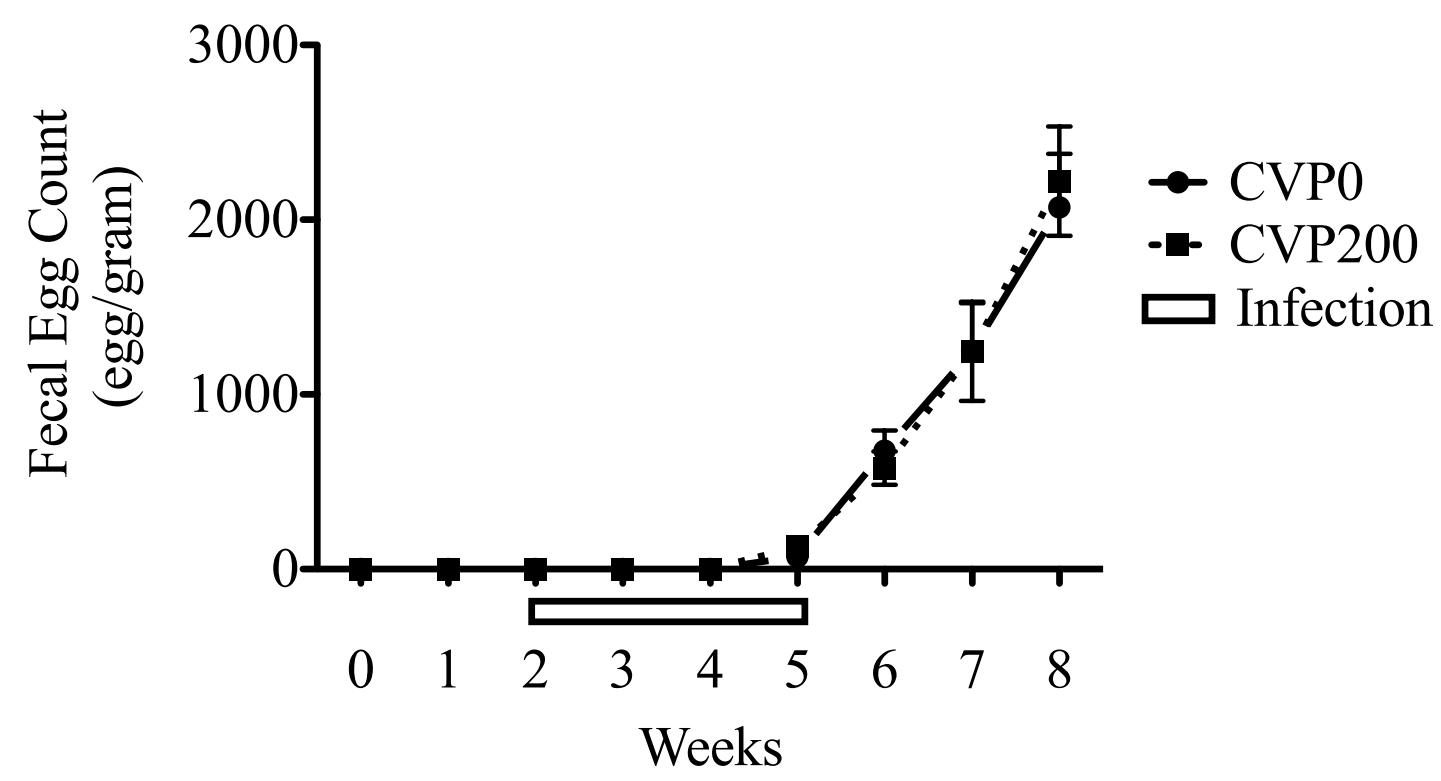

Figure 8. CVP Trial: Effect of cranberry vine pellet (CVP) on fecal egg counts of an experimental trickle infection of Haemonchus contortus in lambs. Lambs were fed cranberry vine pellet (CVP) starting on day 0 and experimentally infected with $H$. contortus (500 L3 three times a week for 3 weeks) starting on day 14 and ending on day 28. Lambs in treatment group CVP200 $(\mathrm{n}=7)$ were fed $500 \mathrm{~g}$ of a $40 \% \mathrm{CV}$ pellet, to consume 200 grams of CV per day. Lambs in control group, CVP0 $(n=7)$, were fed $400 \mathrm{~g}$ grain. Both groups were fed for eight weeks and on day 56, lambs were sent to the abattoir. There was no significant difference between CVP0 and CVP200 groups. Mean \pm SEM.

Average body weight gain over the 8-week trial between CVP0 (178 \pm 49 g/day) and CVP200 (153 $\pm 49 \mathrm{~g}$ /day) groups was not significantly different. When testing for fixed effects across the eight weeks, there was no significant difference by 
treatment $(p=0.81)$ or treatment*week $(p=0.07)$, but there was a significant difference by week $(p<0.0001)$ (Figure not shown).

\subsubsection{Effect of CVP on worm burden, larval burden, and fecundity}

In the CVP trial, the mean number of worms recovered from the control diet lambs was $1054 \pm 162$ worms, and the CVP200 had a mean of $1170 \pm 146$ worms $(p=$ 0.61). There was no difference between the percentage of females in the CVP0 and CVP200 groups (56 \pm 3 vs $52 \pm 4 ; p=0.45$ ). Using the final fecal egg counts of the lambs before they were sent to the abattoir, the fecundity value of the CVP0 group was $4.6 \pm 0.4$ eggs/female and the fecundity value of the CVP200 group was $3.8 \pm 0.6$ eggs/female ( $p=0.28)$ (Figure not shown). Effect of CVP on larval burden was not included in the results, as counts in soaks of control animals were insignificant, and consequently not completed.

\section{Discussion}

The objective of this study was to evaluate the effects of feeding cranberry vine on the worm populations of infected lambs. This was directed at complementing results of a previous study (Barone et al., in preparation; Chapter 3) in which in vitro results testing cranberry vine PAC proved efficacious against eggs, larvae and adult $H$. contortus. In the first study, there was an in vivo trial, however cranberry vine powder was only fed for three days. For this study, it was redesigned to feed the cranberry vine for the full duration of the study as a feed supplement.

Sustainability of feeding cranberry vine

Pruning is one method that can be used for management in the production of cranberries (Suhayda et al., 2009), allowing the canopy to open up and encourage new 
growth (Marucci, 1987). In the fall, cranberry bogs are flooded and the cranberry fruit are harvested from the bogs. Once harvest is over, the bogs are then drained or allowed to freeze over the winter. The snow melts away in the spring and the cranberry producers prune the bogs, cutting down the cranberry vines and removing the prunings. Other than the sale of vines for replanting (Sandler and DeMoranville, 2009), the majority of what is cut goes to waste and is placed into compost piles. The pruning of the cranberry plant vines does not interfere with the harvesting of the cranberries and could be an affordable option for livestock producers to control GIN infection.

A preliminary hurdle faced when feeding any forage to a small ruminant is palatability. The chopped cranberry vine used in the CV trial, initially posed a challenge as the lambs would consume the vine but not finish what was offered to them. The chopped alfalfa hay, mixed with molasses was used to enhance the palatability of the chopped cranberry vine. By using this mixture, feeding up to $200 \mathrm{~g}$ of the cranberry vine was achieved by mixing with $50 \mathrm{~g} \mathrm{AH}$. This was promising, considering that proanthocyanidins can affect food quality because of their bitterness (Monagas et al., 2010) and can sometimes be unappetizing and inedible to small ruminants. The $40 \%$ pellet fed to the lambs was effortlessly consumed, comparable to other successful administrations of PAC containing forage pellets, one example being sericea lespedeza (Kommuru et al., 2015). The ease of attaining cranberry vines, as well as administering it to lambs provides a potentially sustainable product for small ruminant producers to use as an alternative method of GIN control. 


\section{Parasitological parameters}

This investigation examined two different approaches for the use of CV against GIN infections. For the CV trial, the chopped cranberry vine was tested against a fully matured and established infection. Infection development and severity were comparable to other studies (Paolini et al., 2003; Peña et al., 2006; Taylor et al., 2007). The CVP trial tested the cranberry vine against the establishment of a trickle infection, similar to what an animal on pasture would be exposed to. This infection level was consistent with that used by Shaik et al. (2006) in goats.

For both the CV and CVP trials, there were no significant differences in any of the measured parameters, including FEC, PCV, worm burden, and fecundity values. Although there was no statistical difference, the slight suppression in FEC at weeks four and five of the trial might coincide with the higher PCV values of the CV200 group at these weeks, as well as the overall lower worm burden at week five. Other than immune response, the increase in the CV200 group PCV may indicate some type of damage to the worms in which they weren't able to feed as well or reproduce as successfully, keeping the FEC lower. In the case of the previously mentioned study feeding sericea lespedeza (SL) hay to goats, along with the FEC reduction, there was a consequent increase in PCV and lower worm burden observed in the group consuming SL (Shaik et al., 2006) unlike this investigation of cranberry vine. Investigators feeding PAC-rich sainfoin, found fecundity reduced by $60 \%$ compared to the control group (Gaudin et al., 2016), while investigators drenching sheep with PAC containing

Quebracho extract, found fecundity reduced in T. colubriformis and N. battus but not in T. circumcincta and H. contortus worms (Athanasiadou et al., 2001). This finding 
supports this study of $\mathrm{CV}$ and the conclusion that $H$. contortus fecundity was not affected by the administration of $\mathrm{CV}$ to the lambs. The finding that $H$. contortus fecundity was affected by administration of sainfoin (Gaudin et al., 2016), but not by Quebracho (Athanasiadou et al., 2001) or CV (all containing PAC), suggests that there may be a difference in PAC structure and its bioactivity against female fecundity.

When creating the diets for the trials, amounts were based on the assumption of feeding for the heaviest lamb of the 21 lambs $(60 \mathrm{~kg})$ in the CV trial and the heaviest of the 14 lambs in the CVP trial $(60 \mathrm{~kg})$. The targeted average body weight gain was $250 \mathrm{~g}$ /day (National Research Council (NRC), 2007) and actual body weight gains were comparable. The weights changed by week as the lambs continued to grow.

Although the CVP trial allowed us to investigate a different type of infection, there was no significant difference in any of the measured parameters, including FEC, PCV, worm burden, and fecundity values. A similar study that fed tannin-rich sainfoin hay (Onobrychis viciifolia) to lambs used an experimental trickle infection of H. contortus and T. colubriformis to mimic a pasture infection, and observed decreased egg excretion, higher PCV values, decreased total worm numbers, and lower fecundity values (Arroyo-Lopez et al., 2014). These results were not observed in either trial using CV against a matured infection or CVP against a trickle infection. Since this study did not show efficacy against a trickle infection, and showed only a slight suppression in FEC against a fully matured infection, future studies for the use of cranberry vine against $H$. contortus infections in lambs will investigate the potential of feeding the $\mathrm{CV}$ in greater quantities. Based on the amount of PAC present in the 
cranberry vine, future studies should adjust to providing a higher amount of PAC in the volume of $\mathrm{CV}$ fed. The amount of $\mathrm{CV}$ fed needs to meet the nutritional requirements of the animal and consider how much $\mathrm{CV}$ the animal will actually be willing or able to consume. Other studies have fed SL hay by offering the hay in excess (Lange et al., 2006), but using this method of feeding would create difficulty in determining how much PAC is actually consumed by an individual animal.

The CV used in this study contained $190.6 \mathrm{mg}$ PAC/g CV or $19.1 \%$ PAC (cranberry PAC equivalents). It was estimated that administration of $200 \mathrm{~g} /$ day of chopped CV would contain $38.12 \mathrm{~g}$ PAC/day. Assuming an $8 \mathrm{~L}$ rumen capacity, an administration of $38.12 \mathrm{~g}$ PAC/day to each lamb would be equivalent to $4.8 \mathrm{mg}$ $\mathrm{PAC} / \mathrm{mL}$ in the rumen per day. In our previous in vitro investigation of $\mathrm{CV}$, adult worm mortality was $\geq 91 \%$ in concentrations $\geq 0.6 \mathrm{mg} \mathrm{PAC} / \mathrm{mL}$ using isolated PAC extracts. Using CV aqueous extracts, 100\% adult worm mortality was observed in concentrations containing $\geq 3.0 \mathrm{mg} \mathrm{PAC} / \mathrm{mL}$ (Barone et al., in preparation; Chapter 3). Assuming the $\mathrm{CV}$ aqueous extract was the most relevant exposure to what the nematode would encounter in vivo, a concentration of $4.8 \mathrm{mg} \mathrm{PAC} / \mathrm{mL}$ in the rumen was greater than the $3.0 \mathrm{mg} \mathrm{PAC} / \mathrm{mL}$ reported efficacious in vitro. It should be noted that this concentration of PAC in vivo is only an estimate, as other feeds or liquids ingested might add to the total volume in the rumen and dilute this estimated concentration. Additionally, the ability of the estimated amount of PAC extractable in vivo may be much lower than what is extracted in the aqueous extract. For the CVP trial, we were able to administer $500 \mathrm{~g}$ of the $40 \%$ pellet to each lamb daily, ultimately providing the $200 \mathrm{~g} /$ day of chopped $\mathrm{CV}$, consequently administering the same amount 
of PAC daily (38.12 g PAC/day) in both feeding trials. In a study investigating the use of SL against $H$. contortus infections in sheep, the SL used in the study was analyzed for PAC content and contained 22.4\% (quebracho equivalents) (Lange et al., 2006), comparable to the percentage of PAC (19.1\%) in the CV. The difference between the study feeding SL hay and this investigation of feeding CV and CVP, is that the lambs that were fed the SL hay were offered the hay in excess (Lange et al., 2006). Therefore it is difficult to compare the amount of PAC actually consumed by the sheep. The trial indicated that the lambs were supplemented with $0.23 \mathrm{~kg}$ finishing ration daily (Lange et al., 2006), therefore if it was estimated that a four-month old ewe lamb was consuming $0.91 \mathrm{~kg} / \mathrm{lamb} / \mathrm{day}$ hay with the finishing ration, the lamb would ultimately consume $204 \mathrm{~g}$ of PAC (0.91 kg x 0.224), 5.4-fold more PAC consumed than in this investigation of $\mathrm{CV}$. This difference in PAC consumption may explain why the investigation of SL showed efficacy against $H$. contortus, where CV did not.

\section{CV trial versus CVP trial}

One of the main differences between the $\mathrm{CV}$ trial and the CVP trial was that the CV trial investigated an established infection, while the CVP trial investigated the establishment of an infection. While no significant effects were observed on the establishment of incoming $\mathrm{L} 3 \mathrm{H}$. contortus using $\mathrm{CVP}$, feeding the $\mathrm{CV}$ against an established infection showed a slight suppression in the CV200 group FEC at weeks three, four, and five. Supporting these findings, are previously reported studies investigating the use of sericea lespedeza (SL) against these two types of infections (Lange et al., 2006). Feeding SL to sheep, revealed a possible lethal effect on an 
established worm burden, but did not have the same effect on the establishment of incoming L3 (Lange et al., 2006).

Another substantial difference between the CV trial and CVP trial was that the CV trial used chopped cranberry vine and the CVP trial pelleted the CV before administration to the sheep. Pelleting feed increases the feasibility of transport, storage and administration, as well as reduces waste (Terrill et al., 2007). Although pelleting of $\mathrm{CV}$ provided an easier product to administer, there was no anthelmintic efficacy observed by the pelleted CV, while there was a slight suppression observed by the chopped CV. From these findings, it is suspected that the pelleting process may have had an effect on PAC within the CVP. The process of pelleting involves heating (Hoover et al., 2014) to form the pellet, which could potentially have an effect on the $\mathrm{CV}$ and the structure of the PAC. It has been reported that PAC shifts from extractable to bound forms as a result of drying and heating PAC containing forages (Terrill et al., 1990; Terrill et al., 1992; Terrill et al 2007). As a consequence of heating, proanthocyanidins can form covalent bonds with proteins through oxidative polymerization reactions (Reed, 1995). Since PAC has the potential to complex under high heat conditions, this could potentially change the structure of PAC and ultimately reduce its bioactivity (Terrill et al., 2007).

An investigation comparing the efficacy of feeding pelleted SL hay and feeding ground SL hay revealed that pelleting the SL hay enhanced its anthelmintic effectiveness against GIN (Terrill et al., 2007). Although these findings disagree with the comparisons made in this investigation of CV, Terrill et al (2007) also stated that voluntary consumption of the SL pellet was higher than of the ground SL hay and that 
the pellet-fed goats likely consumed a greater total amount of PAC; possibly producing the observed increase in anthelmintic efficacy of the pellets. In this study of CV, the lambs were fed equivalent amounts ( $200 \mathrm{~g} /$ day) of chopped cranberry in either chopped or pelleted form. It was concluded that there may be a limit to the extent that feed can be heated and still maintain its anthelmintic properties (Terrill et al., 2007) and that the chemical structure of PAC and its reactivity may be more important than the amounts of extractable and bound forms (Shaik et al., 2006).

\section{Potential mechanism of action}

Previous research observing $H$. contortus under SEM found accumulation of aggregate on the anterior end of the worms from an in vitro 12-hour incubation with cranberry vine PAC extract (12 mg PAC/mL) (Barone et al., in preparation; Chapter

3). Other investigators observed adult worms from an in vitro 24-hour incubation with organic extracts of tannin rich legume browses including, tzalam (Lysiloma latisiliquum) and sainfoin (Onobrychis viciifolia) at concentrations of $1.2 \mathrm{mg} / \mathrm{mL}$ (Martínez-Ortíz-de-Montellano et al., 2013) and observed similar effects. The study also investigated adult worms exposed to those same PAC containing forages, in vivo (fed to goats for seven days) and a similar accumulation of aggregate was observed on the anterior end of the worms exposed to tzalam under in vivo conditions (MartínezOrtíz-de-Montellano et al., 2013). This accumulation around the anterior end could be due to the tannins' ability to bind to proteins. The intestinal surface of L4 and adult $H$. contortus worms is lined by a helical polymeric structure; that is attached to the luminal surface of the intestinal cells and fills the spaces between the microvilli (Munn, 1977; Geldhof and Knox, 2008). This proline-rich protein, termed contortin, 
is used by the parasite to effectively ingest blood without coagulation. With the ability to bind to proteins, the tannins may have the ability to bind around secretory areas, such as the anterior end, impairing the anticoagulant activity, causing consequential intestinal blockage, or even starvation of the worm (Hoste et al., 2012).

The worms that were exposed to five weeks of feeding chopped cranberry vines did not reveal an accumulation of aggregate at the anterior end, but rather a cracking effect of the cuticle of the worm. Cracking in the cuticle can be caused by the critical point drying process done in the preparation of the worms for SEM, which can introduce significant morphological artifacts (Paterson et al, 2013). Although cracking of the nematode cuticle can commonly occur during sample preparation, in our case, the deep cracking was only observed in the worms from the CV200 group. The CV200 group consistently showed greater cracking and could be indicative of a compromised cuticle due to the contact with the proanthocyanidins.

The lower number of vesicles seen internal to the microvilli of the lining of the lumen of the intestines could be a possible indicator of poor food absorption and an effect on the feeding ability of the CV200 worms. Nematodes rely greatly on lipids for energy storage and metabolism (Behm, 2002) and the intestines appear to be a major site of these biochemical activities. Of the several vacuolar ATPase subunits highly expressed in the intestine, the VHA-6 protein is said to be involved in nutrient uptake. This protein, localized to the apical surface of the intestine, acidifies the intestinal lumen (Knight and Behm, 2012; McGhee, 2007). The suggestion of poor food absorption could be the effect of the worms' ability to feed, but could also be related to one of the many intestinal proteases or peptidases responsible for the 
biochemical activities of digestion, metabolism, and storage. The TEM work on the worms exposed to $\mathrm{CV}$ in vivo investigated the apical domain and the microvilli. The microvillar brush border is the most prominent feature of the apical domain (McGhee, 2007). Gene products and other markers are localized to the apical domain, making this structure of importance. There was no visual difference between the microvilli of the worms from the control group or CV group, as both were well developed. The only difference observed were the lower amount of vesicles internal to the microvilli, leading to the speculation that nutrient uptake was affected.

\section{Conclusions}

Cranberry vines may have the potential to be part of an integrated parasite control program for small ruminants. This study demonstrated that $200 \mathrm{~g}$ chopped CV daily did not have the ability to completely clear an established infection, such as a commercial dewormer does, and pelleted CV did not have the ability to prevent the establishment of an infection. Further studies will investigate the potential of feeding cranberry vine at higher doses for longer periods of time.

\section{Acknowledgements}

The authors of this paper would like to thank Dr. John Porter at A.D. Makepeace Company for providing and assisting with the collection of cranberry vines. Thanks to Paul Avery at Cornell University for his assistance with the grinding of the cranberry vine. The authors would like to thank Nick Miniter at the University of Rhode Island's Peckham Farm and farm staff for the care and housing of the lambs used for this study. The authors would like to thank Kathy Lowe at Virginia Tech for 
her expertise and training in scanning and transmission electron microscopy. The authors also extend their gratitude to Dr. James Baldwin at University of California, Riverside for his expertise in analyzing nematode transmission electron micrographs. This work was supported in part by OREI award number AWD03605 and USDA NE SARE graduate student grant GNE14-071-27806. 


\section{References}

Acharya, M., Burke, J.M., Coffey, K.P., Kegley, E.B., Miller, J.E., Huff, G.R., Smyth, E., Terrill, T.H., Mosjidis, J.A., Rosenkrans, C., 2015. Changes in hematology, serum biochemistry, and gastrointestinal nematode infection in lambs fed sericea lespedeza with or without dietary sodium molybdate. J. Anim. Sci. 93 (4), 1952$1961,10.2527 /$ jas.2014-8584 [doi].

Arroyo-Lopez, C., Manolaraki, F., Saratsis, A., Saratsi, K., Stefanakis, A., Skampardonis, V., Voutzourakis, N., Hoste, H., Sotiraki, S., 2014. Anthelmintic effect of carob pods and sainfoin hay when fed to lambs after experimental trickle infections with Haemonchus contortus and Trichostrongylus colubriformis. Parasite 21, 71, 10.1051/parasite/2014074 [doi].

Athanasiadou, S., Kyriazakis, I., Jackson, F., Coop, R.L., 2001. Direct anthelmintic effects of condensed tannins towards different gastrointestinal nematodes of sheep: in vitro and in vivo studies. Vet. Parasitol. 99 (3), 205-219, S03044017(01)00467-8 [pii].

Barone, C., Zajac, A., Manzi-Smith, L., Howell, A., Reed, J., Krueger, C., Petersson, K. 2017. Anthelmintic efficacy of cranberry vine aqueous and proanthocyanidin extract on ovine Haemonchus contortus. Dissertation; Chapter 3. Manuscript in preparation.

Behm, C. 2002. 'Metabolism', in Donald L Lee (ed.), The Biology of Nematodes, Taylor \& Francis Group, London, pp. 261-290.

Desrues, O., Pena-Espinoza, M., Hansen, T.V., Enemark, H.L., Thamsborg, S.M., 2016. Anti-parasitic activity of pelleted sainfoin (Onobrychis viciifolia) against 
Ostertagia ostertagi and Cooperia oncophora in calves. Parasit. Vectors 9 (1), 329-016-1617-z, 10.1186/s13071-016-1617-z [doi].

Feliciano, R.P., Shea, M.P., Shanmuganayagam, D., Krueger, C.G., Howell, A.B., Reed, J.D., 2012a. Comparison of isolated cranberry (Vaccinium macrocarpon Ait.) proanthocyanidins to catechin and procyanidins A2 and B2 for use as standards in the 4-(dimethylamino) cinnamaldehyde assay. J. Agric. Food Chem. 60 (18), 4578-4585.

Feliciano, R.P., Krueger, C.G., Shanmuganayagam, D., Vestling, M.M., Reed, J.D., 2012b. Deconvolution of matrix-assisted laser desorption/ionization time-offlight mass spectrometry isotope patterns to determine ratios of A-type to Btype interflavan bonds in cranberry proanthocyanidins. Food Chem. 135 (3), 1485-1493, 10.1016/j.foodchem.2012.05.102 [doi].

Feliciano, R.P., Boeres, A., Massacessi, L., Istas, G., Ventura, M.R., Nunes Dos Santos, C., Heiss, C., Rodriguez-Mateos, A., 2016. Identification and quantification of novel cranberry-derived plasma and urinary (poly)phenols. Arch. Biochem. Biophys. 599, 31-41, 10.1016/j.abb.2016.01.014 [doi].

Gaudin, E., Simon, M., Quijada, J., Schelcher, F., Sutra, J.F., Lespine, A., Hoste, H., 2016. Efficacy of sainfoin (Onobrychis viciifolia) pellets against multi resistant Haemonchus contortus and interaction with oral ivermectin: Implications for onfarm control. Vet. Parasitol. 227, 122-129, 10.1016/j.vetpar.2016.08.002 [doi]. Geldhof, P., Knox, D., 2008. The intestinal contortin structure in Haemonchus contortus: an immobilised anticoagulant? Int. J. Parasitol. 38 (13), 1579-1588, 10.1016/j.ijpara.2008.05.002 [doi]. 
Heckendorn, F., Haring, D.A., Maurer, V., Senn, M., Hertzberg, H., 2007. Individual administration of three tanniferous forage plants to lambs artificially infected with Haemonchus contortus and Cooperia curticei. Vet. Parasitol. 146 (1-2), 123-134, S0304-4017(07)00052-0 [pii].

Hoover, A.N., Tumuluru, J.S., Teymouri, F., Moore, J., Gresham, G., 2014. Effect of pelleting process variables on physical properties and sugar yields of ammonia fiber expansion pretreated corn stover. Bioresour. Technol. 164, 128-135, 10.1016/j.biortech.2014.02.005 [doi].

Hoste, H., Jackson, F., Athanasiadou, S., Thamsborg, S.M., Hoskin, S.O., 2006. The effects of tannin-rich plants on parasitic nematodes in ruminants. Trends Parasitol. 22 (6), 253-261, S1471-4922(06)00099-7 [pii].

Hoste, H., Martinez-Ortiz-De-Montellano, C., Manolaraki, F., Brunet, S., OjedaRobertos, N., Fourquaux, I., Torres-Acosta, J.F., Sandoval-Castro, C.A., 2012. Direct and indirect effects of bioactive tannin-rich tropical and temperate legumes against nematode infections. Vet. Parasitol. 186 (1-2), 18-27, 10.1016/j.vetpar.2011.11.042 [doi].

Katiki, L.M., Ferreira, J.F., Gonzalez, J.M., Zajac, A.M., Lindsay, D.S., Chagas, A.C.S., Amarante, A.F., 2013. Anthelmintic effect of plant extracts containing condensed and hydrolyzable tannins on Caenorhabditis elegans, and their antioxidant capacity. Vet. Parasitol. 192 (1), 218-227.

Knight, A.J., Behm, C.A., 2012. Minireview: the role of the vacuolar ATPase in nematodes. Exp. Parasitol. 132 (1), 47-55, 10.1016/j.exppara.2011.09.004 [doi]. 
Kommuru, D.S., Whitley, N.C., Miller, J.E., Mosjidis, J.A., Burke, J.M., Gujja, S., Mechineni, A., Terrill, T.H., 2015. Effect of sericea lespedeza leaf meal pellets on adult female Haemonchus contortus in goats. Vet. Parasitol. 207 (1-2), 170175, 10.1016/j.vetpar.2014.11.008 [doi].

Krueger, C.G., Chesmore, N., Chen, X., Parker, J., Khoo, C., Marais, J.P., Shanmuganayagam, D., Crump, P., Reed, J.D., 2016. Critical reevaluation of the 4-(dimethylamino) cinnamaldehyde assay: cranberry proanthocyanidin standard is superior to procyanidin A2 dimer for accurate quantification of proanthocyanidins in cranberry products. Journal of Functional Foods 22, 13-19.

Krueger, C.G., Reed, J.D., Feliciano, R.P., Howell, A.B., 2013. Quantifying and characterizing proanthocyanidins in cranberries in relation to urinary tract health. Analytical and bioanalytical chemistry 405 (13), 4385-4395.

Lange, K.C., Olcott, D.D., Miller, J.E., Mosjidis, J.A., Terrill, T.H., Burke, J.M., Kearney, M.T., 2006. Effect of sericea lespedeza (Lespedeza cuneata) fed as hay, on natural and experimental Haemonchus contortus infections in lambs. Vet. Parasitol. 141 (3-4), 273-278, S0304-4017(06)00346-3 [pii].

Martínez-Ortíz-de-Montellano, C., Arroyo-López, C., Fourquaux, I., Torres-Acosta, J.F.J., Sandoval-Castro, C.A., Hoste, H., 2013. Scanning electron microscopy of Haemonchus contortus exposed to tannin-rich plants under in vivo and in vitro conditions. Exp. Parasitol. 133 (3), 281-286, http://dx.doi.org/10.1016/j.exppara.2012.11.024.

Marucci, P., 1987. A rationale for the pruning of cranberries. Cranberries 51 (4), 3-10. 
McGhee, J.D., 2007. The C. elegans intestine. WormBook , 1-36, 10.1895/wormbook.1.133.1 [doi].

Miller, J.E. Procedure for recovery of GI parasites. Unpublished laboratory protocol.

Molan, A.L., Faraj, A.M., 2010. The effects of condensed tannins extracted from different plant species on egg hatching and larval development of Teladorsagia circumcincta (Nematoda: Trichostrongylidae). Folia. Parasitol. (Praha) 57 (1), 62-68.

Monagas, M., Quintanilla-Lopez, J.E., Gomez-Cordoves, C., Bartolome, B., LebronAguilar, R., 2010. MALDI-TOF MS analysis of plant proanthocyanidins. J. Pharm. Biomed. Anal. 51 (2), 358-372, 10.1016/j.jpba.2009.03.035 [doi].

Munn, E.A., 1977. A helical, polymeric extracellular protein associated with the luminal surface of Haemonchus contortus intestinal cells. Tissue Cell 9 (1), 2334, 0040-8166(77)90046-5 [pii].

Niezen, J.H., Robertson, H.A., Waghorn, G.C., Charleston, W.A., 1998. Production, faecal egg counts and worm burdens of ewe lambs which grazed six contrasting forages. Vet. Parasitol. 80 (1), 15-27, S0304-4017(98)00202-7 [pii].

National Research Council (NRC), 2007. Nutrient Requirements of Small Ruminants, 1 st ed. National Academy Press, Washington, DC, pp. 256-257.

Paolini, V., Bergeaud, J.P., Grisez, C., Prevot, F., Dorchies, P., Hoste, H., 2003. Effects of condensed tannins on goats experimentally infected with Haemonchus contortus. Vet. Parasitol. 113 (3-4), 253-261, S0304401703000645 [pii].

Paterson, S.M., Casadio, Y.S., Brown, D.H., Shaw, J.A., Chirila, T.V., Baker, M.V., 2013. Laser scanning confocal microscopy versus scanning electron microscopy 
for characterization of polymer morphology: Sample preparation drastically distorts morphologies of poly (2-hydroxyethyl methacrylate)-based hydrogels. J Appl Polym Sci 127 (6), 4296-4304.

Pena, M.T., Miller, J.E., Horohov, D.W., 2006. Effect of CD4+ T lymphocyte depletion on resistance of Gulf Coast Native lambs to Haemonchus contortus infection. Vet. Parasitol. 138 (3-4), 240-246, S0304-4017(06)00049-5 [pii].

Polashock, J., Zelzion, E., Fajardo, D., Zalapa, J., Georgi, L., Bhattacharya, D., Vorsa, N., 2014. The American cranberry: first insights into the whole genome of a species adapted to bog habitat. BMC Plant. Biol. 14, 165-2229-14-165, 10.1186/1471-2229-14-165 [doi].

Reed, J.D., 1995. Nutritional toxicology of tannins and related polyphenols in forage legumes. J. Anim. Sci. 73 (5), 1516-1528.

Sandler, H.A., DeMoranville, C.J., 2009. Economic analysis of nitrogen rate on vine production and fruit yield of pruned cranberry beds. HortTechnology 19 (3), $572-579$.

Shaik, S.A., Terrill, T.H., Miller, J.E., Kouakou, B., Kannan, G., Kaplan, R.M., Burke, J.M., Mosjidis, J.A., 2006. Sericea lespedeza hay as a natural deworming agent against gastrointestinal nematode infection in goats. Vet. Parasitol. 139 (1-3), 150-157, S0304-4017(06)00142-7 [pii].

Skantar, A.M., Agama, K., Meyer, S.L., Carta, L.K., Vinyard, B.T., 2005. Effects of geldanamycin on hatching and juvenile motility in Caenorhabditis elegans and Heterodera glycines. J. Chem. Ecol. 31 (10), 2481-2491, 10.1007/s10886-0057114-z [doi]. 
Suhayda, B., DeMoranville, C.J., Sandler, H.A., Autio, W.R., Heuvel, J.E.V., 2009. Sanding and pruning differentially impact canopy characteristics, yield, and economic returns in cranberry. HortTechnology 19 (4), 796-802.

Taylor, M.A., Coop, R.L., Wall, R.L., 2007. Veterinary Parasitology. $3^{\text {rd }}$ ed. Blackwell Publishing, Ames, IA, pp. 159.

Terrill, T., Rowan, A., Douglas, G., Barry, T., 1992. Determination of extractable and bound condensed tannin concentrations in forage plants, protein concentrate meals and cereal grains. J. Sci. Food Agric. 58 (3), 321-329.

Terrill, T., Windham, W., Evans, J., Hoveland, C., 1990. Condensed tannins in sericea lespedeza: Effect of preservation method on tannin concentration. Crop Sci. 30, 219-224.

Terrill, T.H., Dykes, G.S., Shaik, S.A., Miller, J.E., Kouakou, B., Kannan, G., Burke, J.M., Mosjidis, J.A., 2009. Efficacy of sericea lespedeza hay as a natural dewormer in goats: Dose titration study. Vet. Parasitol. 163 (1-2), 52-56, http://dx.doi.org/10.1016/j.vetpar.2009.04.022.

Terrill, T.H., Mosjidis, J.A., Moore, D.A., Shaik, S.A., Miller, J.E., Burke, J.M., Muir, J.P., Wolfe, R., 2007. Effect of pelleting on efficacy of sericea lespedeza hay as a natural dewormer in goats. Vet. Parasitol. 146 (1-2), 117-122, S03044017(07)00083-0 [pii].

Vargas-Magana, J.J., Torres-Acosta, J.F., Aguilar-Caballero, A.J., Sandoval-Castro, C.A., Hoste, H., Chan-Perez, J.A., 2014. Anthelmintic activity of acetone-water extracts against Haemonchus contortus eggs: interactions between tannins and 
other plant secondary compounds. Vet. Parasitol. 206 (3-4), 322-327, S03044017(14)00535-4 [pii].

Veríssimo, C.J., Niciura, S.C.M., Alberti, A.L.L., Rodrigues, C.F.C., Barbosa, C.M.P., Chiebao, D.P., Cardoso, D., da Silva, G.S., Pereira, J.R., Margatho, L.F.F., da Costa, R.L.D., Nardon, R.F., Ueno, T.E.H., Curci, V.C.L.M., Molento, M.B., 2012. Multidrug and multispecies resistance in sheep flocks from São Paulo state, Brazil. Vet. Parasitol. 187 (1-2), 209-216, http://dx.doi.org/10.1016/j.vetpar.2012.01.013.

Whitlock, H.V., 1948. Some modifications of the McMaster Helminth egg-counting technique and apparatus. J. Counc. Sci. Res. 21, pp. 177-180. 


\section{CHAPTER 5}

\section{Conclusions}

Birdsfoot trefoil (BFT, Lotus corniculatus) and cranberry vine (CV, Vaccinium macrocarpon) both have shown in vitro potential to be used as effective alternatives to control Haemonchus contortus infections in the northeastern United States. Both plants can be grown natively and can be made palatable to small ruminants. Birdsfoot trefoil can be grazed or harvested and fed as hay, while cranberry vine proved to be palatable when mixed with chopped alfalfa hay or incorporated into a $40 \%$ pellet. The birdsfoot trefoil strains as well as cranberry vine showed anthelmintic efficacy in vitro. In addition, cranberry vine showed promise in vivo, with a slight suppression in fecal egg counts after feeding chopped cranberry vine, but did not confirm anthelmintic potential. As with many other investigated forages, this anthelmintic efficacy is promising, but the mechanism of action remains unknown.

The method by which PAC exerts efficacy against $H$. contortus is undetermined and prompted discussion throughout these presented studies. Testing the anthelmintic efficacy of $\mathrm{CV}$ and BFT extracts in vitro, coupled with the phytochemical analyses of the extracts, initiated discussion on PAC content and structure, different secondary compounds present in the extracts, and if any or all of these variables are contributing to anthelmintic efficacy. For all of the in vitro testing, purified PAC extracts and crude aqueous extracts were tested in parallel assays, and anti-parasitic activity was compared. In the majority of assays, CV and BFT aqueous extracts showed different efficacy compared to PAC extracts. These studies exemplified the difficulty in describing how PAC exerts harmful effects on $H$. 
contortus. From interpreting results across all three studies investigating BFT and $\mathrm{CV}$, the mode of action might be best described as multifaceted. The first part of this multilayered discussion was that the amount of PAC the parasite was exposed to increased efficacy, since dose dependent efficacy was observed using both CV and BFT purified PAC extracts. Many investigators, including Brunet et al. (2007), have found dose dependent responses to PAC extracts, supporting this finding.

The second concept was that secondary compounds found in the CV aqueous extract contributed to the in vitro efficacy observed, since the $\mathrm{CV}$ aqueous extract was just as efficacious at much lower levels of PAC as compared to the purified CV PAC extract. In some cases, the aqueous extract exhibited efficacy (at comparable PAC concentrations), while the purified PAC extract did not. In the CV in vitro studies, the presence of other secondary compounds in the aqueous extract was confirmed. This lead to the theory, that other secondary compounds in the crude aqueous extracts were exerting a synergistic effect with PAC. This hypothesis is supported by previously published studies, making similar conclusions about secondary compounds (Klongsiriwet et al., 2015; Acharya et al., 2014; Hernández-Villegas et al., 2011; Monteiro et al., 2011).

The third concept was recognized in cases where the same extract was used at equivalent concentrations against different developmental stages of $H$. contortus. In the majority of cases, at the same concentrations, there was a difference in effectiveness when comparing anthelmintic efficacy in ovicidal, larvicidal, and adult assays. This lead to the conclusion that the same concentration of extract might not exert the same efficacy, depending on what stage of the parasite is exposed to the CV 
or BFT extract. The parasite might be better protected at certain developmental stages, or the mechanism of action by which the extract exerts deleterious effects is not able to do so because of the structure and composition of the parasite at that life stage. Supporting this hypothesis, previous studies have also recognized that the effect that PAC-rich plants have on gastrointestinal parasites varies with the parasites' stage of development (Hoste et al., 2006).

The fourth concept was that the structure of the PAC influenced bioactivity. When comparing the purified CV-PAC extract to the crude CV aqueous extract, there was no difference between the structures (linkages, glycosylation, hydroxylation) of the PAC present in both extracts. This was expected, since the extracts were made using the same CV powder from the same plant. When looking at the BFT investigation, which investigated 51 different strains of the same species, a difference in structure might explain the difference in bioactivity. This type of structurally influenced bioactivity has been observed in PAC linkages, where A-type linkages in PAC proved to exhibit greater bioactivity than B-type PAC linkages, specifically against Escherichia coli (Feliciano et al., 2014). Since the diversity in PAC content and efficacy of the aqueous extracts was not correlated, it suggests that if there is a correlation, it might exist with structural characteristics or the presence of other secondary compounds.

This multidimensional interpretation of the in vitro investigations of $\mathrm{CV}$ and BFT, which included secondary compounds, PAC content and structure, and the developmental stage of the parasite, is not a new idea in this field of research. Similarly, previous investigators have stated that the effects that PAC-rich forages 
have on gastrointestinal parasites vary with the parasite, its stage of development, and the biochemical characteristics of the plant species (Hoste et al., 2006). The complexities within each of these factors might be why the mechanism of action is still unknown and is what makes this type of investigation challenging. Further investigation of specific developmental stages will be important to rule out specific questions from these studies, such as why $\mathrm{CV}$ aqueous extract inhibited egg hatch and caused L1 mortality, but at the same concentrations, did not inhibit exsheathment or cause L3 mortality. Additional work is needed to determine secondary compounds present in crude aqueous extracts and to compare to purified PAC, addressing the contrasting results as to why BFT aqueous extracts inhibited exsheathment, and BFT purified PAC did not. Further investigations using purified PAC from more than just one BFT variety is necessary to determine if there is also varietal anthelmintic diversity when using isolated PAC in bioassays, to eliminate any synergistic effects from other secondary compounds present in the aqueous extracts, and to determine if PAC structure is an influential factor in anthelmintic efficacy of PAC-containing forages.

In vitro, we can compare the BFT-AqE to the efficacy of the CV-AqE using $\mathrm{LC}_{50}$ concentrations. Based solely on the efficacy of the extracts against egg hatching, the 51 BFT strains were $50 \%$ efficacious at an average concentration of $2.8 \mathrm{mg} / \mathrm{mL} \pm$ 0.3 (mean $\pm \mathrm{SEM}$ ), compared to the $\mathrm{CV}-\mathrm{AqE}$ that was $50 \%$ efficacious at a concentration of $5.3 \mathrm{mg} / \mathrm{mL}$. Based on these results we could assume that BFT might be a better option for producers to feed to their animals. We can also compare the Bruce-PAC to the CV-PAC using $\mathrm{LC}_{50}$ concentrations. Based on the efficacy of the 
purified PAC against egg hatching, the Bruce-PAC would be 50\% efficacious at a concentration of $1795.0 \mathrm{mg} / \mathrm{mL}$, while the CV-PAC would be $50 \%$ efficacious at 26.6 $\mathrm{mg} / \mathrm{mL}$. In this comparison of PAC, we might suggest $\mathrm{CV}$ as the better option for producers to use against GIN infections.

Aside from the in vitro bioassays and the implications from those results, there were a few other investigative measures regarding mechanism of action, focusing solely on the adult worm. Incubating adults in vitro in both CV-PAC and CV-AqE showed an accumulation of aggregate on the cuticle, and around the buccal area. It was concluded from these findings that the $\mathrm{CV}$ might be having an effect on the motility of the worm and its ability to feed. From the in vivo trials, which showed suppression in fecal egg counts after feeding CV at weeks 3, 4, and 5, worms were collected from lambs fed chopped CV and a cracking effect was observed on the cuticle of the worm as well as decreased number of vesicles internal to microvilli lining of the intestines. Observations made from both in vitro and in vivo observations, concluded that PAC might be exerting an effect on the cuticle and an effect on the ability of the adult to feed, possibly leading to malnutrition and consequently, a decreased number of vesicles. Using florenscently labeled PAC, to observe any possible binding on the cuticle or internally, could further examine this effect. These studies warrant further investigations of adult $H$. contortus exposed to PAC in vivo to determine if feeding higher amounts of $\mathrm{CV}$ would result in similar aggregate accumulation seen in these $\mathrm{CV}$ in vitro studies and reported in other previous published studies (Martínez-Ortíz-de-Montellano et al, 2013). 
In summary, further investigation regarding the use of BFT and CV as an alternative method of GIN control in small ruminants is necessary. Feeding higher amounts of $\mathrm{CV}$ to lambs, investigating the extracts for other secondary compounds, comparing the structures of $\mathrm{PAC}$ in the BFT varieties and identifying any correlations would all contribute to further our knowledge of this study. Although there was a slight suppression in fecal egg count observed when feeding chopped cranberry, this treatment was not comparable to a commercial anthelmintic and could not be used in the same way. Further testing using higher doses of $\mathrm{CV}$ would provide further information. Determining the role of PAC against different developmental stages in vitro will assist in determining how PAC exerts anthelmintic efficacy. Further testing would ultimately be beneficial to determine promising forages or varieties to feed to animals and help control GIN infections. In the future, with much investigation, it would be advantageous to be able to recommend forages, based on PAC content and/or structural characteristics, to producers to plant for grazing or harvesting as hay. If producers could sustainably grow or feasibly acquire PAC-containing forages that reduced incidences of $H$. contortus related losses, and reduced producer reliance on commercial anthelmintics, this would have a positive impact on the small ruminant industry, both environmentally and economically. 


\section{References}

Acharya, M., Burke, J.M., Coffey, K.P., Kegley, E.B., Miller, J.E., Huff, G.R., Smyth, E., Terrill, T.H., Mosjidis, J.A., Rosenkrans, C., 2015. Changes in hematology, serum biochemistry, and gastrointestinal nematode infection in lambs fed sericea lespedeza with or without dietary sodium molybdate. J. Anim. Sci. 93 (4), 1952-1961, 10.2527/jas.2014-8584 [doi].

Brunet, S., Aufrere, J., El Babili, F., Fouraste, I., Hoste, H., 2007. The kinetics of exsheathment of infective nematode larvae is disturbed in the presence of a tanninrich plant extract (sainfoin) both in vitro and in vivo. Parasitology 134 (Pt 9), 12531262, S0031182007002533 [pii].

Feliciano, R.P., Meudt, J.J., Shanmuganayagam, D., Krueger, C.G., Reed, J.D., 2014. Ratio of "A-type" to "B-type" proanthocyanidin interflavan bonds affects extra-intestinal pathogenic Escherichia coli invasion of gut epithelial cells. J. Agric. Food Chem. 62 (18), 3919-3925, 10.1021/jf403839a [doi].

Hernández-Villegas, M.M., Borges-Argáez, R., Rodriguez-Vivas, R.I., Torres-Acosta, J.F.J., Méndez-Gonzalez, M., Cáceres-Farfan, M., 2011. Ovicidal and larvicidal activity of the crude extracts from Phytolacca icosandra against Haemonchus contortus. Vet. Parasitol. 179 (1-3), 100-106, http://dx.doi.org/10.1016/j.vetpar.2011.02.019.

Hoste, H., Jackson, F., Athanasiadou, S., Thamsborg, S.M., Hoskin, S.O., 2006. The effects of tannin-rich plants on parasitic nematodes in ruminants. Trends Parasitol. 22 (6), 253-261, S1471-4922(06)00099-7 [pii]. 
Klongsiriwet, C., Quijada, J., Williams, A.R., Mueller-Harvey, I., Williamson, E.M., Hoste, H., 2015. Synergistic inhibition of Haemonchus contortus exsheathment by flavonoid monomers and condensed tannins. Int. J. Parasitol. Drugs Drug Resist 5 (3), 127-134, 10.1016/j.ijpddr.2015.06.001 [doi].

Martínez-Ortíz-de-Montellano, C., Arroyo-López, C., Fourquaux, I., Torres-Acosta, J.F.J., Sandoval-Castro, C.A., Hoste, H., 2013. Scanning electron microscopy of Haemonchus contortus exposed to tannin-rich plants under in vivo and in vitro conditions. Exp. Parasitol. 133 (3), 281-286, http://dx.doi.org/10.1016/j.exppara.2012.11.024.

Monteiro, M.V.B., Bevilaqua, C.M.L., Morais, S.M., Machado, L.K.A., CamurçaVasconcelos, A.L.F., Campello, C.C., Ribeiro, W.L.C., Mesquita, M.d.A., 2011. Anthelmintic activity of Jatropha curcas L. seeds on Haemonchus contortus. Vet. Parasitol. 182 (2-4), 259-263, http://dx.doi.org/10.1016/j.vetpar.2011.04.010. 


\begin{abstract}
APPENDIX
Standard Operating Procedure for Blood Collection

(Adapted protocol from the thesis of Caitlyn MacGlaflin; modified from Purdue

University Blood Sampling in Sheep by Mitchell et al. (Retrieved on September 23, 2008 from http://www.ces.purdue.edu/extmedia/AS/AS-557-W.pdf))

Blood Collection:
\end{abstract}

1. Properly restrain animal holding the head parallel to the ground and at a $30^{\circ}$ angle to the side.

2. Electric clippers may be used to prepare the neck by shaving off a patch of wool approximately 4 inches wide and 8 inches long.

3. To locate the vein, apply pressure with the thumb approximately half way down the side of the neck (on either side).

4. Secure a Vacutainer needle $\left(21 \mathrm{G}, 1 \frac{1}{2} 2\right.$ inch needle, Becton, Dickinson, and Company; Franklin Lakes, NJ, USA; REF \#367215) to the Vacutainer holder (Becton, Dickinson, and Company; Franklin Lakes, NJ, USA; REF \#364815) by twisting.

5. While holding off on the vein with one hand, insert the needle into the vein with the bevel facing upward.

6. Once the needle is placed, push the EDTA tube (Becton, Dickinson, and Company; Franklin Lakes, NJ, USA; REF \#367863) into the holder and onto the needle. 
7. Gently adjust the needle until blood begins to flow into the tube. Blood flow will cease once the tube is full.

8. Gently remove the tube and slowly invert eight times.

9. Remove the needle and your hand from the neck and allow the vein to relax. 10. EDTA tubes should be immediately placed in ice. 


\section{Standard Operating Procedure for Determining Packed Cell Volume}

(Adapted protocol from the thesis of Caitlyn MacGlaflin)

Sample Preparation:

1. Collect blood by jugular venipuncture into Vacutainer EDTA tubes, invert tube eight times, and place on ice.

2. Invert tube to mix before use.

3. Without spilling, tip tube so that blood moves toward the opening. Place one end of the micro hematocrit capillary tube (Thermo Fisher Scientific Inc., Waltham, MA, USA; REF \#22-362-574) into the blood and allow tube to fill by capillary action. Only fill tube up to $3 / 4$ of the way.

4. Place fingertip at one end of the tube to prevent leaking. Insert the other end of the tube into the white sealing wax (Critoseal, Leica Biosystems, Buffalo Grove, IL, USA; REF \#02-676-20).

5. Place capillary tubes into centrifuge rotor, making sure that the wax in the end of the tube faces outward (not towards the center of the centrifuge). All samples should be run in duplicate and placed across from each other, keeping the centrifuge balanced.

6. To prevent tubes from cracking, take a pin and gently push tubes against the outer edge of the rotor.

7. Centrifuge for 3 minutes at $15,000 \mathrm{RPM}$ at room temperature.

Sample Reading:

1. Spin the PCV Reader (International Equipment Company; Needham, MA) until it reaches the 100 mark. 
2. Place the capillary tube into the sample groove.

3. Align the wax/blood interface with the line at the bottom of the sample groove.

4. Spin the top plate of the PCV reader until the "swirl" aligns with the plasma/air interface.

5. Spin both plates until the "swirl" aligns with the white blood cell/red blood cell interface. The value under the red line is the PCV to be read and reported as a percentage. 


\section{Standard Operating Procedure for Fecal Egg Counts (FEC)}

(Whitlock (1948), Modified McMaster Technique)

\section{Procedure:}

1. Fecal samples are collected directly from the rectum and kept refrigerated until analysis. Samples should be run as promptly as possible, but within seven days from the time of collection.

2. Two grams of feces are measured on a calibrated scale and placed into a 1-2 ounce cup.

3. Add $28 \mathrm{ml} \mathrm{Fecasol}{ }^{\circledR}$ (Vetoquinol U.S.A., Inc., Fort Worth, TX, USA; REF \#014008-1G) to feces and soak for approximately 5 minutes.

4. Gently break up fecal pellet with a tongue depressor. Set cup aside to sit for approximately 5 minutes.

5. Pour fecal solution through a square of 2-ply cheesecloth into a new cup. Use tongue depressor to gently press fecal solution through gauze.

6. Wet the McMaster slide (Chalex Corporation, Issaquah, WA, USA) with distilled water and gently pat dry top and bottom with paper towels.

7. Immediately pipet solution into both sides of the McMaster slide, using a $1 \mathrm{ml}$ syringe, a sample of the suspension and fill one side of the chamber.

8. Place slide on microscope platform and let sit, without disturbance, for 5 minutes.

9. Focus on the top layer using the low power (10x) objective. Count all eggs inside of the grid areas (greater than $1 / 2$ of egg inside grid).

10. Count only trichostrongylid eggs (oval shaped, $\sim 80-90$ microns long) 
11. Total egg count:

$($ chamber $1+$ chamber 2$) * 50=$ eggs per gram $($ epg $)$ 


\section{Standard Operating Procedure for Nematode Recovery Post Mortem}

(Adapted protocol from Miller parasitology laboratory, unpublished methods)

Preparation:

1. Lambs are stunned by captive bolt and exsanguinated.

2. Gastrointestinal tract is collected as soon as possible following exsanguination.

3. Mesenteric tissue is cleaned away from the abomasum and three pieces of butcher's rope are used to tie the junction of the omasum and abomasum, the junction of the abomasum and beginning of the small intestine, and at the junction of the small intestine and large intestine.

4. The rumen, reticulum, and omasum are removed and the remaining is placed in a cooler until nematode recovery begins.

Nematode Recovery:

Abomasum Wash:

1. The abomasum is removed from the tract and cleaned of excess fat and mesenteric tissue.

2. A scalpel is used to open the abomasum along the greater curvature.

3. Contents are collected into a pan by washing with warm water. The folds of the abomasum are gently rubbed to remove any debris.

4. Once completely cleaned the abomasal contents are brought up to $2 \mathrm{~L}$.

5. The contents are mixed thoroughly using an inverted beaker and stirring in a figure eight motion. Immediately a $10 \%$ aliquot of the contents, $200 \mathrm{ml}$, is taken and placed into a $500 \mathrm{ml}$ jar. 
6. The sample is fixed with $200 \mathrm{ml} 10 \%$ phosphate buffered formalin (PBF; Thermo Fisher Scientific Inc., Waltham, MA, USA; REF \#SF100-20) and stored until nematode quantification and identification.

\section{Abomasum Soak:}

(A soak is performed to collect any larvae that may be embedded within the tissue of the abomasum.)

1. Once the wash is completed, the abomasum is spread out on in a pan, covered with saline.

2. The abomasum is incubated at $37^{\circ} \mathrm{C}$ overnight.

3. The contents of the soak are collected and the abomasum is gently washed with saline and collected.

4. Contents are allowed to settle for at least 2 hours.

5. In order to collect the settled contents a serological pipet is used to remove as much liquid without disturbing the bottom as possible.

6. The remaining sample is collected into $500 \mathrm{ml}$ jars, $200 \mathrm{ml}$ in each, and fixed with $200 \mathrm{ml} 10 \%$ PBF each.

7. Using a No. 200 sieve, larvae will be removed from the $5 \%$ PBF and backwashed off the sieve with water.

8. Nematodes will be pipetted onto a petri dish (with $13 \mathrm{~mm}$ grid, BD Bioscience) and stained with Lugol's Iodine (Lugol's Iodine, Electron Microscopy Sciences, Hatfield, PA, USA; REF \#26055-05). Sodium hypochlorite will be added to allow for counting of larvae using a 
dissection microscope (Zoom Stereomicroscope, Nikon ${ }^{\circledR}$ SMZ745, MVI, Avon, MA).

9. Adults will be identified by sex and stage of development. 


\section{Standard Operating Procedure for Egg Recovery}

(Adapted protocol from Hoste parasitology laboratory, unpublished methods)

Procedure:

1) Obtain 8-10 grams of feces from animal with more than 2,000 epg.

2) Place feces in small cup (4oz/120cc).

3) Activate hatchability.

a) Add enough water to break up the feces.

b) Mush with hands. End with slurry.

c) Rinse hand.

4) Rinse through $1 \mathrm{~mm}$ sieve

a) Place sieve over empty bucket (11 liter bucket).

b) Pour mixture through sieve, rubbing with hand to increase speed.

c) Rub and rinse through with tap water until clear.

d) Rinse sieve in sink.

e) Run mixture through sieve again without rinsing, discard remaining debris.

5) Repeat step 4 through the $355 \mu \mathrm{m}$ and $150 \mu \mathrm{m}$ sieve.

6) Rinse through the $38 \mu \mathrm{m}$ sieve but do not discard solids collected in sieve. This now contains eggs. Use water to rinse the solids off the sieve into an empty bucket.

7) Repeat step 6 through the $25 \mu \mathrm{m}$ sieve. Use $50 \mathrm{ml}$ Fecasol ${ }^{\circledR}$ to rinse solids into large glass beaker. 
8) Use transfer pipets to evenly distribute egg mixture into four $15 \mathrm{ml} \mathrm{Falcon}{ }^{\mathrm{TM}}$ tubes (Corning ${ }^{\mathrm{TM}}$ Falcon $^{\mathrm{TM}}$ 50mL Conical Centrifuge Tubes, Corning Life Sciences, Tewksbury, MA, USA; REF \#352070). Using Fecasol®, bring each volume to the top of the tube to form a positive meniscus. Carefully place a cover slip on the top of the tube and place in the centrifuge.

9) Using centrifuge, spin tubes at $200 \mathrm{x}$ g for 2 minutes.

10) Slowly remove cover slips by lifting straight up off the top of the tubes and rinse with water over the $25 \mu \mathrm{m}$ sieve.

11) Wash off the $25 \mu \mathrm{m}$ sieve with $14 \mathrm{~mL}$ of tap water into beaker. Pipette into $15 \mathrm{ml}$ Falcon ${ }^{\mathrm{TM}}$ tube.

12) Using the micropipetter, measure out $20 \mu \mathrm{L}$ and put on slide. Set up a ratio to determine how much is needed to get 100 eggs.

Ex: if there are 69 eggs in $20 \mu \mathrm{L}$

$$
69 \text { eggs } / 20 \mu \mathrm{L}=100 \text { eggs } / \mathrm{X} \mu \mathrm{L}
$$

Ratio determines $\mu$ L's needed for next step of egg hatch assay. 


\section{Standard Operating Procedure for Egg Hatch and L1 Mortality Assay}

(Adapted protocol from Assis et al. (2003) and Marie-Magdeleine et al. (2009); in vitro methods)

Procedure:

1) Weigh out desired amount of either PAC or plant material. Create stock solution using tap water, determining the stock solution concentration to be double of the highest concentration in the wells. Prepare serial dilutions of extracts using tap water.

a. PAC extracts:

i. Weigh out $\mathrm{mg}$ of PAC and dissolve in $14 \mathrm{~mL}$ tap water

ii. Example: $33.6 \mathrm{mg}$ PAC dissolved into $14 \mathrm{~mL}=2.4 \mathrm{mg} / \mathrm{mL}$ concentration (to be used as $1.2 \mathrm{mg} / \mathrm{mL}$ in well)

b. AqE extracts:

i. Weigh out mg of powder and add volume with tap water up to $30 \mathrm{~mL}, 24$ hours prior to use in assay

ii. Example: $360 \mathrm{mg}$ soaked in $30 \mathrm{~mL}=12 \mathrm{mg} / \mathrm{mL}$ concentration (to be used as $6 \mathrm{mg} / \mathrm{mL}$ in well)

iii. After 24 hours, centrifuge the plant material to the bottom of the $50 \mathrm{~mL}$ Falcon ${ }^{\mathrm{TM}}$ tube and use the supernatant as the extract.

2) Make control solution using Thiabendazole (TBZ, Thermo Fisher Scientific Inc., Waltham, MA, USA) in dimethyl sulfoxide (DMSO, Fisher BioReagents $^{\mathrm{TM}}$, Thermo Fisher Scientific Inc., Waltham, MA, USA; REF 
\#BP231-1) at a concentration of $1 \mathrm{mg}$ TBZ/mL DMSO (1.4 mg TBZ dissolved in $1400 \mu \mathrm{L}$ DMSO).

3) Obtain a concentration of eggs from recovery. Correct concentration of egg solution to be approximately 100 eggs in $100 \mu 1$.

4) Add 100 eggs (in $100 \mu l$ ) per well into a 24-well plate (Corning ${ }^{\mathrm{TM}}$, Falcon ${ }^{\mathrm{TM}}$, Polystyrene Microplates, Corning Life Sciences, Tewksbury, MA, USA; REF \#353226).

5) Designate negative controls by only adding tap water to wells. Add $10 \mu 1$ of the TBZ stock solution to each positive control well (in a total volume of $2 \mathrm{~mL}$ this will give a final concentration of $0.5 \mu \mathrm{g} / \mathrm{mL}$ of TBZ).

6) For wells that did not get $10 \mu \mathrm{l}$ of TBZ solution, add $10 \mu \mathrm{l}$ of DMSO.

7) All wells receive $890 \mu \mathrm{l}$ tap water increasing the volume to $1 \mathrm{~mL}$.

8) Add either prepared PAC extract solutions $(1 \mathrm{ml})$ or add $1 \mathrm{ml}$ water to negative or positive control wells, increasing to total volume of $2 \mathrm{~mL}$.

9) Incubate for 24 hours at $26^{\circ} \mathrm{C}$.

10) Count number of eggs and live larvae in each well (based on 5 second observation of motility) at 24 hours.

11) At 24 hours, kill plate in heated water bath $\left(55^{\circ} \mathrm{C}\right.$ for 10 minutes $)$.

12) Count number of eggs and larvae. 


\section{Standard Operating Procedure for L3 Mortality and Exsheathment Inhibition}

\section{Assay}

(Adapted protocol from Conder and Johnson (1996), in vitro methods)

Procedure:

1) Weigh out desired amount of either PAC or plant material. Create stock solution using tap water, determining the stock solution concentration to be double of the highest concentration in the wells. Prepare serial dilutions of extracts using tap water.

a. PAC extracts:

i. Weight out $\mathrm{mg}$ of PAC and dissolve in $14 \mathrm{~mL}$ tap water

ii. Example: $33.6 \mathrm{mg}$ PAC dissolved into $14 \mathrm{~mL}=2.4 \mathrm{mg} / \mathrm{mL}$ concentration (to be used as $1.2 \mathrm{mg} / \mathrm{mL}$ in tubes).

b. AqE extracts:

i. Weight out mg of powder and add volume with tap water up to $30 \mathrm{~mL}, 24$ hours prior to use in assay

ii. Example: $360 \mathrm{mg}$ soaked in $30 \mathrm{~mL}=12 \mathrm{mg} / \mathrm{mL}$ concentration (to be used as $6 \mathrm{mg} / \mathrm{mL}$ in tubes)

iii. After 24 hours, centrifuge the plant material to the bottom of the $50 \mathrm{~mL}$ Falcon ${ }^{\mathrm{TM}}$ tube and use the supernatant as the extract.

2) Obtain $L_{3}$ infective larvae, less than 3 months old.

3) Stock solutions of either PAC or AqE extracts are made fresh prior to each assay and serially diluted using water. 
4) Sheathed L3 larvae (2,000) are added to Earle's Balanced Salt Solution (EBSS, Sigma-Aldrich®, Inc., Natick, MA, USA; REF \#E3024) up to a volume of $1 \mathrm{~mL}$ in a $15 \mathrm{~mL}$ Falcon ${ }^{\mathrm{TM}}$ tube.

5) Tap water $(1 \mathrm{~mL})$ will be added to water control larvae, and $1 \mathrm{~mL}$ of extract will be added to treated larvae, for a total volume of $2 \mathrm{~mL}$ in the Falcon $^{\mathrm{TM}}$ tube.

6) Larvae will incubate at $38^{\circ} \mathrm{C}$ for 24 hours, and checked for viability.

7) Parafilm M® (Bemis Company, Inc., Neenah, WI; REF \#HS234526A-1) is used to stretch over the top of the tubes as a cover. A glass pipet tip, connected to a $\mathrm{CO}_{2}$ tank, is carefully placed down into the larval solution by puncturing through the Parafilm $M \circledR$. Making sure the Parafilm $M \circledR$ is still stretched.

8) $\mathrm{CO}_{2}$ is then bubbled into tubes for 15 minutes immediately prior to the incubation period.

9) Caps are screwed on each tube for incubation after removing the $\mathrm{CO}_{2}$ feeder tube.

10) Tubes are then placed back into the incubator at $38^{\circ} \mathrm{C}$ for $18-24$ hours.

11) Following the 18-hour incubation, percent exsheathment and percent viability are determined by averaging counts of exsheathed larvae from the first 100 motile worms observed. 


\section{Standard Operating Procedure for Adult Worm Assay}

(Adapted protocol from Kotze and McClure (2001), in vitro methods)

\section{Procedure:}

1) Infected lambs are sent to the abattoir.

2) Stomachs are collected from lambs with high fecal egg counts.

a. Ask slaughterhouse manager to tie off both ends of stomach and bring out to you.

b. Place on dissection tray, find other end of abomasum, tie off and then cut away from omasum.

c. Transfer the abomasa in gallon sized plastic bags, in warm cooler (warmed by keeping hot water in the cooler prior to receiving the stomachs).

3) When abomasa are at the lab, using a scalpel, slice open, along the greater curvature to pick worms.

4) Using wire pickers, pick worms from the folds of the abomasum.

5) Place directly into warm $\left(37^{\circ} \mathrm{C}\right)$ phosphate buffered saline (PBS) in a culture dish.

6) Make stock solutions:

a. Amphotericin B Stock Solution $(0.25 \mathrm{mg} / \mathrm{mL})$

i. $2.5 \mathrm{mg}$ Amphotericin B (Sigma-Aldrich ${ }^{\circledR}$, Inc., Natick, MA, USA; REF \#A9528)

ii. 10 mL RPMI-1640 (Fisher Chemical ${ }^{\mathrm{TM}}$, Thermo Fisher Scientific Inc., Waltham, MA, USA; REF \#12-167F) 
b. Streptomycin/Penicillin Stock Solution $(10,000 \mathrm{U} / \mathrm{mL}$ Penicillin, 10 $\mathrm{mg} / \mathrm{mL}$ Streptomycin

7) Make 1-2 hour holding medium:

i. $\quad 0.8 \%$ glucose (Dextrose (D-glucose) Anhydrous (Granular Powder/Cerified ACS), Fisher Chemical ${ }^{\mathrm{TM}}$, Thermo Fisher Scientific Inc., Waltham, MA, USA; REF\#50-99-7)

ii. $\quad 2.5 \mu \mathrm{g} / \mathrm{mL}$ Amphotericin B

iii. $\quad 100 \mathrm{U} / \mathrm{mL}$ Penicillin and

iv. $100 \mu \mathrm{g} / \mathrm{mL}$ Streptomycin (Corning ${ }^{\mathrm{TM}}$ cellgro $^{\mathrm{TM}}$ PenicillinStreptomycin Solution, Corning Life Sciences, Tewksbury, MA, USA)

v. $10 \mathrm{mM} \mathrm{N}$-[2-hydroxyethyl0piperazine-N-[4-butanesulfonic acid] (HEPES) buffer (pH 6.8) (Fisher Chemical ${ }^{\mathrm{TM}}$, Thermo Fisher Scientific Inc., Waltham, MA, USA; REF \#BP310100)

a. For $200 \mathrm{~mL}$ 10x (antibacterial/antifungal) solution preparation:

i. To $180 \mathrm{~mL}$ RPMI-1640 add:
a. 196 mL RPMI-1640
b. $2 \mathrm{~mL}$ Strep/Pen Stock Solution
c. $2 \mathrm{~mL}$ Amphotericin B Stock Solution
d. $1.6 \mathrm{~g}$ Glucose
e. $476.6 \mathrm{mg}$ HEPES 
ii. Bring up to volume $(200 \mathrm{~mL})$ with RPMI.

8) Fill a new culture dish with warm $\left(37^{\circ} \mathrm{C}\right)$ incubation solution. Transfer worms into this dish, leaving behind any stomach material to clean them.

9) Let the worms incubate in these for $1-2$ hours in a $\mathrm{CO}_{2}$ incubator $\left(38^{\circ} \mathrm{C}\right.$, $\left.5 \% \mathrm{CO}_{2}\right)$.

During Incubation, set up for Adult Worm Assay

1) Set up 10 replicates of each concentration of PAC, AqE, water, and TBZ control.

2) Make TBZ control stock solution:

a. $\quad 0.05 \mathrm{mg} / \mathrm{mL}$ TBZ (in wells) (Dissolved 25mg TBZ in $1 \mathrm{~mL}$ DMSO).

3) Make Incubation Medium:

a. Medium used for 48 hour incubation:

i. $0.8 \%$ glucose

ii. $10 \mathrm{mmol}$ HEPES

iii. $\quad 0.25 \mu \mathrm{g} / \mathrm{mL}$ Amphotericin B

iv. $10 \mathrm{U} / \mathrm{mL}$ Penicillin

v. $10 \mu \mathrm{g} / \mathrm{mL}$ Streptomycin

b. For $200 \mathrm{~mL} \mathbf{1 x}$ (antibacterial/antifungal) solution preparation:

i. To $180 \mathrm{~mL}$ RPMI-1640 add:

1. 199.6 mL RPMI- 1640

2. $200 \mu \mathrm{L}$ Strep/Pen Stock Solution

3. $200 \mu \mathrm{L}$ Amphotericin B Stock Solution 
4. $1.6 \mathrm{~g}$ Glucose

5. $476.6 \mathrm{mg}$ HEPES

ii. Bring up to volume (200 mL) with RPMI-1640

*HEPES buffer - molecular weight $=238.3 \mathrm{~g} / \mathrm{mol}$

4) Weigh out desired amount of either PAC or plant material. Create stock solutions and serial dilutions using RPMI-1640 incubation medium. Adjust for what the concentration will be in the well.

5) Place adults into individual wells and let incubate at $38^{\circ} \mathrm{C}, 5 \% \mathrm{CO}_{2}$ for 48 hours. Check larvae at 24 and 48 hours. Record number of worms dead and alive and note if they are alive, but sluggish. 


\section{References}

Assis, L.M., Bevilaqua, C.M.L., Morais, S.M., Vieira, L.S., Costa, C.T.C., Souza, J.A.L., 2003. Ovicidal and larvicidal activity in vitro of Spigelia anthelmia Linn. extracts on Haemonchus contortus. Vet. Parasitol. 117 (1-2), 43-49, http://dx.doi.org/10.1016/j.vetpar.2003.07.021.

Conder, G.A., Johnson, S.S., 1996. Viability of infective larvae of Haemonchus contortus, Ostertagia ostertagi, and Trichostrongylus colubriformis following exsheathment by various techniques. J. Parasitol. 82 (1), 100-102.

Kotze, A.C., McClure, S.J., 2001. Haemonchus contortus utilises catalase in defence against exogenous hydrogen peroxide in vitro. Int. J. Parasitol. 31 (14), 15631571, S0020751901003034 [pii].

Marie-Magdeleine, C., Hoste, H., Mahieu, M., Varo, H., Archimede, H., 2009. In vitro effects of Cucurbita moschata seed extracts on Haemonchus contortus. Vet. Parasitol. 161 (1-2), 99-105, 10.1016/j.vetpar.2008.12.008 [doi].

Whitlock, H.V., 1948. Some modifications of the McMaster Helminth egg-counting technique and apparatus. J. Counc. Sci. Res. 21, pp. 177-180. 